\title{
Belief Propagation Guided Decimation Fails on Random Formulas
}

AMIN COJA-OGHLAN, Goethe University, Mathematics Institute

Let $\Phi$ be a uniformly distributed random $k$-SAT formula with $n$ variables and $m$ clauses. Nonconstructive arguments show that $\boldsymbol{\Phi}$ is satisfiable for clause/variable ratios $m / n \leq r_{k-\mathrm{SAT}} \sim 2^{k} \ln 2$ with high probability. Yet no efficient algorithm is known to find a satisfying assignment beyond $m / n \sim 2^{k} \ln (k) / k$ with a nonvanishing probability. On the basis of deep but nonrigorous statistical mechanics ideas, a message passing algorithm called Belief Propagation Guided Decimation has been put forward (Mézard, Parisi, Zecchina: Science 2002; Braunstein, Mézard, Zecchina: Random Struc. Algorithm 2005). Experiments suggested that the algorithm might succeed for densities very close to $r_{k-\mathrm{SAT}}$ for $k=3,4,5$ (Kroc, Sabharwal, Selman: SAC 2009). Furnishing the first rigorous analysis of this algorithm on a nontrivial input distribution, in the present article we show that Belief Propagation Guided Decimation fails to solve random $k$-SAT formulas already for $m / n=O\left(2^{k} / k\right)$, almost a factor of $k$ below the satisfiability threshold $r_{k-\mathrm{SAT}}$. Indeed, the proof refutes a key hypothesis on which Belief Propagation Guided Decimation hinges for such $m / n$.

Categories and Subject Descriptors: G.3 Mathematics of Computing [Probability and Statistics]: Probabilistic Reasoning Algorithms

General Terms: Algorithms, Theory

Additional Key Words and Phrases: Satisfiability, belief propagation, random structures

ACM Reference Format:

Amin Coja-Oghlan. 2017. Belief propagation guided decimation fails on random formulas. J. ACM 63, 6, Article 49 (January 2017), 55 pages.

DOI: http://dx.doi.org/10.1145/3005398

\section{INTRODUCTION AND RESULTS}

Let $k \geq 3$ and $n>1$ be integers, let $r>0$ be a fixed real number (independent of $n$ ), and set $m=\lceil r n\rceil$. Let $\boldsymbol{\Phi}=\boldsymbol{\Phi}_{k}(n, m)$ be a propositional formula obtained by choosing a set of $m$ clauses (without replacement) of length $k$ over the variables $x_{1}, \ldots, x_{n}$ uniformly at random such that no variable occurs in the same clause more than once (either positively or negatively). For $k, r$ fixed we say that $\boldsymbol{\Phi}$ has some property $\mathcal{P}$ with high probability if $\lim _{n \rightarrow \infty} \mathrm{P}[\boldsymbol{\Phi} \in \mathcal{P}]=1$.

\subsection{Background and Motivation}

Since the 1990s, the random formula $\Phi$ has gained a reputation as an extremely challenging benchmark for SAT solving. More precisely, early computer experiments led to two key hypotheses [Taylor et al. 1991; Selman and Kirkpatrick 1994; Levesque et al. 1992]. First, that there is a sharp threshold for satisfiability. That is, for any clause length $k$ there is a threshold value $r_{k-\mathrm{SAT}}>0$ such that the random formula $\Phi$ is satisfiable with high probability if $r<r_{k-S A T}$, whereas $\boldsymbol{\Phi}$ is unsatisfiable with

Supported by EPSRC grant EP/G039070/2. An extended abstract version of this work appeared in the proceedings of the ACM-SIAM Symposium on Discrete Algorithms ('SODA') 2011.

Author's address: A. Coja-Oghlan, Goethe University, Mathematics Institute, 10 Robert Mayer St, Frankfurt 60486, Germany; email: acoghlan@math.uni-frankfurt.de.

\section{(i)}

This work is licensed under a Creative Commons Attribution International 4.0 License.

2017 Copyright is held by the owner/author(s).

ACM 0004-5411/2017/01-ART49 \$15.00

DOI: http://dx.doi.org/10.1145/3005398

Journal of the ACM, Vol. 63, No. 6, Article 49, Publication date: January 2017. 
high probability if $r>r_{k-S A T}$. Second, that standard SAT-solvers such as DPLL-based algorithms require an exponential time to find a satisfying assignment for densities $r$ close to $r_{k-S A T}$. Thus, while these algorithms are highly efficient on "real-world" SAT instances, the simplest conceivable model of random formulas eludes them. These two hypotheses have inspired a considerable amount of research over the years, both experimental and theoretical [Achlioptas 2009]. Moreover, similar phenomena have been hypothesized in many other random problems [Achlioptas et al. 2005].

While the precise values (and even the existence) of the $k$-SAT threshold remain unknown for small $k \geq 3$, the precise thresholds are known for $k$ exceeding a certain (undetermined) constant $k_{0}$ [Sun et al. 2015]. While the precise formula is complicated, the $k$-SAT threshold satisfies $r_{k-\mathrm{SAT}}=2^{k} \ln 2-\frac{1}{2}(1+\ln 2)+\varepsilon_{k}$, where with $\varepsilon_{k} \rightarrow 0$ for large $k$.

With respect to the computational problem, in spite of two decades of extensive research in the CS community no algorithm seemed capable of finding a satisfying assignment for densities $r$ anywhere close to $r_{k-S A T}$ in polynomial time with a nonvanishing probability. More precisely, the best rigorously analyzed polynomial time algorithm, designed specifically to "beat" random formulas, is known to succeed for $r<\left(1-\varepsilon_{k}\right) 2^{k} \ln (k) / k$ with high probability and seems to fail beyond [Coja-Oghlan 2010]. Furthermore, a plethora of algorithms are known to fail asymptotically (in the clause length $k$ ) for even smaller densities $r=\rho \cdot 2^{k} / k$ with $\rho>0$ an absolute constant (independent of $k$ ). Examples include simple linear-time algorithms such as Unit Clause $(\rho=\mathrm{e} / 2)$ [Franco and Chao 1990] or Shortest Clause $(\rho=1.817)$ [Suen and Frieze 1996], as well as a wide range of DPLL-type algorithms ( $\rho=11 / 4)$ [Molloy et al. 2004]. In summary, there remained a factor of about $k / \ln k$ between the satisfiability threshold and the density where algorithms are known to find satisfying assignments efficiently.

Against this gloomy background, it came as a considerable surprise when experiments indicated that certain highly efficient message passing algorithms find satisfying assignments at densities within a whisker of the conjectured satisfiability threshold [Braunstein et al. 2005; Selman and Gomes 2002; Selman et al. 2009; Zecchina et al. 2002]. These algorithms, called Belief Propagation Guided Decimation and Survey Propagation Guided Decimation, were put forward on the basis of the "cavity method," a very insightful but nonrigorous technique from statistical mechanics [Braunstein et al. 2005; Ricci-Tersenghi et al. 2007]. ${ }^{1}$ Conceptually, Belief/Survey Propagation Guided Decimation are more sophisticated than the previously studied algorithms by an order of magnitude; we give a detailed account in Section 1.3. As a consequence, the techniques developed to analyze previous algorithms fail dramatically for Belief/Survey Propagation.

The performance of the new message-passing algorithms can be exemplified nicely in the case $k=4$ (see also Figure 1 ). The conjectured threshold for the existence of satisfying assignments is $r_{4-\mathrm{SAT}} \approx 9.93$ [Zecchina et al. 2006]. According to experiments from Selman et al. [2009], Survey Propagation-guided decimation finds satisfying assignments efficiently for densities up to $r=9.73$. Experiments from Semerjian and Ricci-Tersenghi [2009] suggest that the "vanilla" version of Belief Propagation Guided Decimation succeeds up to $r=9.05$. With a certain tweak (the "most biased variable" decimation rule), Belief Propagation Guided Decimation succeeds up to $r=9.24$ [Selman et al. 2009]. By comparison, the best "classical" algorithm SCB from Suen and

\footnotetext{
${ }^{1}$ The message-passing procedure on which Belief Propagation Guided Decimation is based has been rediscovered several times in the context of different applications; see Section 1.5 for details. In the physics literature, it was originally known under the name "Bethe-Peierls approximation." By contrast, the message-passing technique that underpins Survey Propagation seems to be new.
} 

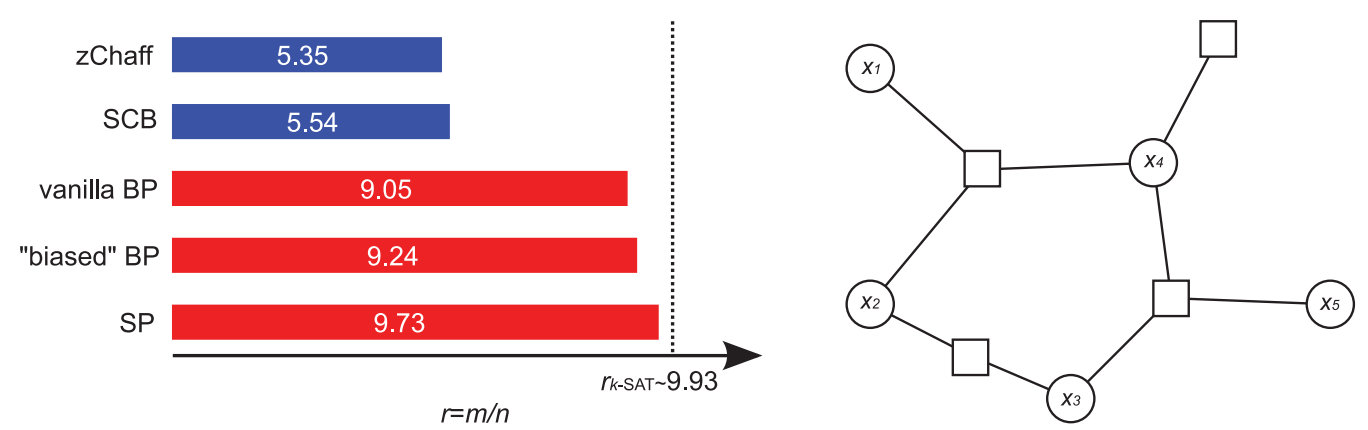

Fig. 1. Left: experimental performance of various algorithms on random 4-SAT. Right: the factor graph of the formula $\left(\neg x_{1} \vee x_{2} \neg x_{4}\right) \wedge\left(\neg x_{2} \vee x_{3}\right) \wedge\left(\neg x_{3} \vee x_{4} \vee \neg x_{5}\right) \wedge \neg x_{4}$, with circles representing variables and squares representing clauses.

Frieze [1996] finds satisfying assignments in polynomial time merely up to $r=5.54$, whereas zChaff, an industrial SAT solver, is effective up to $r=5.35$ [Selman et al. 2009].

\subsection{Unsatisfied with Physics}

Ever since these stunning experimental results were reported, coming up with a rigorous analysis of the new message-passing algorithms has been one of the key challenges in the area of random constraint satisfaction problems (cf. Achlioptas et al. [2005]). The present article contributes the first such analysis. More specifically, we study the "vanilla" version of Belief Propagation Guided Decimation ('BPdec'), the simplest but arguably most natural version. We establish a negative result: BPdec fails to find a satisfying assignment with high probability for densities $r>\rho \cdot 2^{k} / k$ for a certain absolute constant $\rho>0$. In other words, we prove that, perhaps surprisingly, BPdec does not outperform simpler combinatorial algorithms such as the one from Coja-Oghlan [2010] asymptotically.

Stating the result precisely requires a little care because it involves two levels of randomness: the choice of the random formula $\Phi$ and the "coin tosses" of the randomized algorithm BPdec. For a (fixed, nonrandom) $k$-CNF $\Phi$, let success $(\Phi)$ denote the probability that $\operatorname{BPdec}(\Phi)$ outputs a satisfying assignment. Here, of course, "probability" refers to the coin tosses of the algorithm only. Then, if we apply BPdec to the random $k$-CNF $\Phi$, the success probability $\operatorname{success}(\boldsymbol{\Phi})$ becomes a random variable. Recall that $\Phi$ is unsatisfiable for $r>2^{k} \ln 2$ with high probability.

THeOREm 1.1. There is a constant $\rho_{0}>0$ such that for any $k, r$ satisfying

$$
\rho_{0} \cdot 2^{k} / k \leq r \leq 2^{k} \ln 2
$$

we have $\operatorname{success}(\boldsymbol{\Phi}) \leq \exp (-\Omega(n))$ with high probability.

Theorem 1.1 contrasts with the very promising experimental results. The explanation for this is that the experiments were conducted for "small" $k=3,4,5$ [Selman et al. 2009; Semerjian and Ricci-Tersenghi 2009]. Indeed, already for $k=10$ large-scale experiments are difficult to carry out because the relevant density $r$ scales exponentially with $k$. Thus, the good experimental performance can be attributed to the value of the constant $\rho_{0}$ in Theorem 1.1. Because the analysis is intricate as-is, no attempt has been made to compute (or optimize) $\rho_{0}$.

Since Belief/Survey Propagation-guided decimation were suggested [Zecchina et al. 2002], there have been various stabs at explaining the performance of Belief/Survey Propagation Guided Decimation by means of nonrigorous physics arguments 
[Braunstein et al. 2005; Ricci-Tersenghi et al. 2007; Semerjian and Ricci-Tersenghi 2009]. We review this work in more detail in Section 1.4, but, roughly speaking, the predictions were as follows. In chronological order:

- the authors of Braunstein et al. [2005] opined that Belief Propagation Guided Decimation fails for $r>\left(1+\varepsilon_{k}\right) 2^{k} \ln (k) / k$.

- More optimistically, it was predicted in Ricci-Tersenghi et al. [2007] that Belief Propagation Guided Decimation will find satisfying assignments efficiently up to $r \sim 2^{k} \ln 2$.

- Finally, and most pessimistically, according to Semerjian and Ricci-Tersenghi [2009] Belief Propagation Guided Decimation ought to fail for $r>\rho \cdot 2^{k} / k$ for an absolute constant $\rho>0$.

All these predictions derived from fairly sophisticated statistical mechanics reasoning, and both [Ricci-Tersenghi et al. 2007; Semerjian and Ricci-Tersenghi 2009] quote experimental evidence thereby (unintentionally) highlighting the need for a rigorous analysis. Theorem 1.1 confirms the scenario put forward in Semerjian and RicciTersenghi [2009] but does not sit well with the predictions from Ricci-Tersenghi et al. [2007]. Furthermore, the present analysis shows that the reasoning from Braunstein et al. [2005], where the demise of Belief Propagation Guided Decimation was attributed to a certain change in the geometry of the set of satisfying assignments, is off the mark.

A potential objection to a negative result like Theorem 1.1 is that it might hinge on a small detail of the algorithm that could easily be fixed. However, in the sequel, we will see that in the regime our analysis refutes a key hypothesis upon which BPdec depends. In other words, we show that BPdec falls victim to a conceptual issue, not a technicality. Furthermore, some of the arguments used to prove Theorem 1.1 may be of independent interest as they can be expected to extend to applications of BP beyond random $k$-SAT. For instance, we develop a technique for tracing BP on certain quasi-random problem instances.

Finally, we point out that Theorem 1.1 has no immediate bearing on the potentially more powerful Survery Propagation algorithm. We comment on Survey Propagation in Section 1.4.

\subsection{The BPdec Algorithm}

Fix a satisfiable $k$-CNF $\Phi$ on the variables $V=\left\{x_{1}, \ldots, x_{n}\right\}$. We generally represent truth assignments as maps $\sigma: V \rightarrow\{-1,1\}$, with -1 representing false and 1 representing true. (It turns out that using \pm 1 instead of the more common 0,1 simplifies the description of BP quite a bit.) Let $\mathcal{S}(\Phi)$ denote the set of all satisfying assignments of $\Phi$. The algorithm BPdec is an attempt at implementing the following thought experiment:

EXPERIMENT 1.2. Input: A satisfiable $k$-CNF $\Phi$. Result: An assignment $\sigma: V \rightarrow\{-1,1\}$.

0. Let $\Phi_{0}=\Phi$.

1. For $t=0, \ldots, n-1$ do

2. Compute the fraction

$$
M_{x_{t+1}}\left(\Phi_{t}\right)=\frac{\left|\left\{\sigma \in \mathcal{S}\left(\Phi_{t}\right): \sigma\left(x_{t+1}\right)=1\right\}\right|}{\left|\mathcal{S}\left(\Phi_{t}\right)\right|}
$$

of satisfying assignments of $\Phi_{t}$ in which the variable $x_{t+1}$ takes the value 1 .

3. Assign

$$
\sigma\left(x_{t+1}\right)=\left\{\begin{array}{cl}
1 & \text { with probability } M_{x_{t+1}}\left(\Phi_{t}\right) \\
-1 & \text { with probability } 1-M_{x_{t+1}}\left(\Phi_{t}\right)
\end{array}\right.
$$


4. Obtain the formula $\Phi_{t+1}$ from $\Phi_{t}$ by substituting the value $\sigma\left(x_{t+1}\right)$ for $x_{t+1}$ and simplifying, i.e.,

- remove all clauses that got satisfied by setting $x_{t+1}$ to $\sigma\left(x_{t+1}\right)$,

- omit $x_{t}$ from all the other clauses.

5. Return the assignment $\sigma$.

A moment's reflection reveals that this experiment not only produces a satisfying assignment, but that its (random) outcome is in fact uniformly distributed over the set $\mathcal{S}(\Phi)$. We observe that, in the formulas $\Phi_{t}$ obtained at intermediate steps, some clauses can (and typically will) have length less than $k$.

Referring to the successive assignments of variables and the corresponding shrinking of the formula, we call this experiment the decimation process. The obvious obstacle to implementing it is the computation of the marginal probabilities $M_{x_{t+1}}\left(\Phi_{t}\right)$. Indeed, this task is \#P-hard on worst-case inputs.

Yet under what conditions could we hope to compute (or approximate) the marginals $M_{x}\left(\Phi_{t}\right)$ ? Clearly, the marginals are influenced by "local" effects. For instance, if $x$ occurs in a unit clause $a$ of $\Phi_{t}$ (i.e., a clause whose other $k-1$ variables have been assigned already without satisfying $a$ ), then $x$ must be assigned so as to satisfy $a$. Hence, if $x$ appears in $a$ positively, then $M_{x}\left(\Phi_{t}\right)=1$, and otherwise $M_{x}\left(\Phi_{t}\right)=0$. Similarly, if $x$ occurs only positively in $\Phi_{t}$, then $M_{x}\left(\Phi_{t}\right) \geq 1 / 2$. Furthermore, these local effects propagate: If $x$ appears in a clause $a$ whose other variables $y$ are subject to influences from other clauses $b_{y} \neq a$, then the local effects operating on the variables $y$ may impact $x$ via $a$. In the most extreme case, think of a variable $x$ that occurs in a clause $a$ whose other variables are all constrained by unit clauses to take values that fail to satisfy $a$. Then $a$ effectively turns into a unit clause for $x$.

The key hypothesis underlying BPdec is that, in random formulas, such local effects determine the marginals $M_{x}\left(\Phi_{t}\right)$ asymptotically. To define "local" precisely, we need a metric on the variables/clauses. This metric is the shortest path distance on the factor graph $G=G\left(\Phi_{t}\right)$ of $\Phi_{t}$, which is a bipartite graph whose vertices are the variables $V_{t}=\left\{x_{t+1}, \ldots, x_{n}\right\}$ and the clauses of $\Phi_{t}$. Each clause is adjacent to the variables that occur in it (see Figure 1 for an example). For an integer $\omega \geq 1$, let $N^{[\omega]}(x)$ signify the set of all vertices of $G$ that have distance at most $2 \omega$ from $x$. Then the induced subgraph $G\left[N^{[\omega]}(x)\right]$ corresponds to the subformula of $\Phi_{t}^{[\omega]}$ obtained by removing all clauses and variables at distance more than $2 \omega$ from $x_{t}$. Note that all vertices at distance precisely $2 \omega$ are variables. Hence, any satisfying assignment of $\Phi$ induces a satisfying assignment of the subformula. Let us denote by

$$
M_{x}^{[\omega]}\left(\Phi_{t}\right)=M_{x}\left(\Phi_{t}^{[\omega]}\right)=\left|\left\{\sigma \in \mathcal{S}\left(\Phi_{t}^{[\omega]}\right): \sigma(x)=1\right\}\right| /\left|\mathcal{S}\left(\Phi_{t}^{[\omega]}\right)\right|
$$

the marginal probability that $x_{t}$ takes the value 1 in a random satisfying assignment of this subformula.

Of course, in the worst case, the local marginals $M_{x}^{[\omega]}\left(\Phi_{t}\right)$ are just as difficult to compute as the $M_{x}\left(\Phi_{t}\right)$ themselves. But BPdec employs an efficient heuristic called Belief Propagation (BP), which yields certain values $\mu_{x_{t}}^{[\omega]}\left(\Phi_{t}\right) \in[0,1]$; we state this heuristic later. If $G\left[N^{[\omega]}\left(x_{t}\right)\right]$ is a tree, then provably $\mu_{x_{t}}^{[\omega]}\left(\Phi_{t}\right)=M_{x_{t}}^{[\omega]}\left(\Phi_{t}\right)[$ Braunstein et al. 2005]. Moreover, standard arguments show that in a random formula $\Phi$ actually $G\left[N^{[\omega]}\left(x_{t}\right)\right]$ is a tree with high probability so long as $\omega=o(\ln n)$. More generally, in order to obtain an efficient algorithm it would be sufficient for the BP outcomes $\mu_{x_{t}}^{[\omega]}\left(\Phi_{t}\right)$ to approximate the true overall marginals $M_{x_{t}}\left(\Phi_{t}\right)$ well for some (say, polynomially computable, polynomially bounded) function $\omega=\omega(n) \geq 1$. This leads to the following hypothesis underpinning BPdec (cf. Ricci-Tersenghi et al. [2007]). 
HyPOTHESIS 1.3. With probability 1-o(1) over the choice of $\Phi$ and the random decisions in Experiment 1.2 the following holds for all $0 \leq t<n$.

i. For any $\varepsilon>0$ there is $\omega=\omega(\varepsilon, k, r)$ such that $\left|M_{x_{t+1}}\left(\boldsymbol{\Phi}_{t}\right)-M_{x_{t+1}}^{[\omega]}\left(\boldsymbol{\Phi}_{t}\right)\right| \leq \varepsilon$.

ii. For any $\varepsilon>0$ there is $\omega=\omega(\varepsilon, k, r)$ such that $\left|M_{x_{t+1}}\left(\boldsymbol{\Phi}_{t}\right)-\mu_{x_{t+1}}^{[\omega]}\left(\boldsymbol{\Phi}_{t}\right)\right| \leq \varepsilon$.

Hypothesis 1.3 motivates the following algorithm [Semerjian et al. 2007b], which is called Belief Propagation Guided Decimation because it combines BP (Step 2) with a decimation step (Steps 3-4).

Algorithm 1.4. $\operatorname{BPdec}(\Phi)$

Input: $\mathrm{A} k-\mathrm{CNF} \Phi$ on $V=\left\{x_{1}, \ldots, x_{n}\right\}$. Output: An assignment $\sigma: V \rightarrow\{-1,1\}$.

0 . Let $\Phi_{0}=\Phi$.

1. For $t=0, \ldots, n-1$ do

2. Use BP to compute $\mu_{x_{t+1}}^{[\omega]}\left(\Phi_{t}\right)$.

3. Assign

$$
\sigma\left(x_{t+1}\right)=\left\{\begin{array}{cl}
1 & \text { with probability } \mu_{x_{t+1}}^{[\omega]}\left(\Phi_{t}\right), \\
-1 & \text { with probability } 1-\mu_{x_{t+1}}^{[\omega]}\left(\Phi_{t}\right) .
\end{array}\right.
$$

4. Obtain the formula $\Phi_{t+1}$ from $\Phi_{t}$ by substituting the value $\sigma\left(x_{t+1}\right)$ for $x_{t+1}$ and simplifying.

5. Return the assignment $\sigma$.

Remark 1.5. The function $\omega=\omega(k, r, n)$ is "hard-wired" into the preceding algorithm, and our analysis does not depend on any assumptions on $\omega$. In particular, the statement of Theorem 1.1 is understood to hold for all integer-valued functions $\omega=\omega(n) \geq 0$.

Although, strictly speaking, Hypothesis 1.3 provides neither a necessary nor a sufficient condition for BPdec to succeed on random $k$-CNFs with high probability, the hypothesis inspired the algorithm (we will get back to this in Section 1.4). Combining parts of the present analysis of the dynamics of the BP computation (more precisely, Theorem 3.4 below) with techniques for analyzing the geometry of the space of satisfying assignments, we proved the following in Pachon-Pinzon and Coja-Oghlan [2012].

Corollary 1.6. Both statements of Hypothesis 1.3 are false for $k, r$ satisfying (1).

To complete the presentation of the algorithm, we need to define Belief Propagation for $k$-SAT; for a detailed derivation we point the reader to Braunstein et al. [2005], Mézard and Montanari [2009], and Pearl [1988]. Ultimately, we need to define the value $\mu_{x_{t+1}}^{[\omega]}\left(\Phi_{t}\right)$ in Step 2 of BPdec.

Let $N(v)$ denote the neighborhood of a vertex $v$ of the factor graph $G\left(\Phi_{t}\right)$. For a variable $x \in V_{t}$ and a clause $a \in N(x)$ we denote the ordered pair $(x, a)$ by $x \rightarrow a$. Similarly, $a \rightarrow x$ stands for the pair $(a, x)$. Furthermore, we let $\operatorname{sign}(x, a)=1$ if $x$ occurs in $a$ positively, and $\operatorname{sign}(x, a)=-1$ otherwise.

The message space $\mathcal{M}\left(\Phi_{t}\right)$ is the set of all tuples

$$
\left(\mu_{x \rightarrow a}(\zeta)\right)_{x \in V_{t}, a \in N(x), \zeta \in\{-1,1\}}
$$

such that $\mu_{x \rightarrow a}( \pm 1) \in[0,1]$ and $\mu_{x \rightarrow a}(-1)+\mu_{x \rightarrow a}(1)=1$ for all $x, a, \zeta$. For $\mu \in \mathcal{M}\left(\Phi_{t}\right)$ we define $\mu_{a \rightarrow x}(\operatorname{sign}(x, a))=1$ and

$$
\mu_{a \rightarrow x}(-\operatorname{sign}(x, a))=1-\prod_{y \in N(a) \backslash\{x\}} \mu_{y \rightarrow a}(-\operatorname{sign}(y, a)) .
$$


Furthermore, we define the Belief Propagation operator BP as follows: For any $\mu \in \mathcal{M}\left(\Phi_{t}\right)$ we define $\operatorname{BP}(\mu) \in \mathcal{M}\left(\Phi_{t}\right)$ by letting

$$
(\operatorname{BP}(\mu))_{x \rightarrow a}(\zeta)=\frac{\prod_{b \in N(x) \backslash\{a\}} \mu_{b \rightarrow x}(\zeta)}{\prod_{b \in N(x) \backslash\{a\}} \mu_{b \rightarrow x}(-1)+\prod_{b \in N(x) \backslash\{a\}} \mu_{b \rightarrow x}(1)}
$$

unless the denominator equals zero, in which case $(\operatorname{BP}(\mu))_{x \rightarrow a}(\zeta)=\frac{1}{2}$.

Finally, the $\mu_{x}^{[\omega]}\left(\Phi_{t}\right)$ in Steps 2-3 of BPdec are defined as follows. Let $\mu^{[0]}=\frac{1}{2} \cdot \mathbf{1} \in$ $\mathcal{M}\left(\Phi_{t}\right)$ be the vector with all entries equal to $\frac{1}{2}$. Moreover, define inductively $\mu^{[\ell+1]}=$ $\mathrm{BP}\left(\mu^{[\ell]}\right)$ for $0 \leq \ell<\omega$. Then

$$
\mu_{x}^{[\omega]}\left(\Phi_{t}\right)=\frac{\prod_{b \in N(x)} \mu_{b \rightarrow x}^{[\omega]}(1)}{\prod_{b \in N(x)} \mu_{b \rightarrow x}^{[\omega]}(-1)+\prod_{b \in N(x)} \mu_{b \rightarrow x}^{[\omega]}(1)}
$$

for any $x \in V_{t}$, unless the denominator is zero, in which case we set $\mu_{x}^{[\omega]}\left(\Phi_{t}\right)=\frac{1}{2}$.

The intuition here is that the $\mu_{x \rightarrow a}(\zeta)$ are "messages" from a variable $x$ to the clauses $a$ in which $x$ occurs, indicating how likely $x$ was to take the value $\zeta$ if clause $a$ was removed from the formula. Based on these, Equation (2) yields messages $\mu_{a \rightarrow x}(\zeta)$ from clauses $a$ to variables $x$, indicating the probability that $a$ is satisfied if $x$ takes the value $\zeta$ and all other variables $y \in N(a) \backslash\{x\}$ are assigned independently with probability $\mu_{y \rightarrow a}( \pm 1)$. The BP operator in Equation (3) then uses the messages $\mu_{a \rightarrow x}$ in order to "update" the messages from variables to clauses. More precisely, for each $x$ and $a \in N(x)$ the new messages $(\mathrm{BP}(\mu))_{x \rightarrow a}(\zeta)$ are computed under the hypothesis that all other clauses $b \in N(x) \backslash\{a\}$ are satisfied with probabilities $\mu_{b \rightarrow x}(\zeta)$ independently if $x$ takes the value $\zeta$. Finally, the difference between Equations (3) and (4) is that the latter product runs over all clauses $b \in N(x)$. An inductive proof shows that, if for a variable $x$ the subgraph $G\left[N^{[\omega]}(x)\right]$ of the factor graph is acyclic, then in fact $\mu_{x}^{[\omega]}\left(\Phi_{t}\right)=M_{x}^{[\omega]}\left(\Phi_{t}\right)$ [Braunstein et al. 2005].

1.3.1. Variations of the Algorithm. BPdec could be called the "vanilla" version of Belief Propagation Guided Decimation. It is the simplest but arguably the most natural variant. Nonetheless, several other installments have been suggested and experimented with. They differ in how the number $\omega$ of iterations is chosen and how exactly the result of the Belief Propagation calculation is used to decimate.

In the "vanilla" variant, we used an a priori number $\omega$ of iterations. An alternative idea is to iterate the Belief Propagation operator until it reaches a fixed point. More precisely, to accommodate numerical inaccuracies, one could stop after $\omega$ iterations, with $\omega \geq 1$ the least integer such that for some small $\varepsilon>0$ we have

$$
\max _{x \rightarrow a}\left|\mu_{x \rightarrow a}^{[\omega]}(1)-\mu_{x \rightarrow a}^{[\omega-1]}(1)\right|<\varepsilon,
$$

where the maximum is taken over all edges of the factor graph (e.g., Braunstein et al. [2005] and Mézard and Montanari [2009]). Unfortunately, it is not generally assured that the convergence criterion in Equation (5) will ever be met. Hence, one would need to specify how to proceed otherwise. For instance, one could specify an a priori maximum number of iterations. Our analysis can be adapted easily to accommodate these modifications (details omitted). 
More importantly, one could come up with a more sophisticated decimation strategy (i.e., a different way of using the BP result $\mu^{[\omega]}$ to choose the variable to be assigned next and its value). In BPdec, we went for the "vanilla rule": The variables are assigned in the natural order and, each time, the assignment is performed randomly based on the BP estimate of the marginal.

But in experiments a more common decimation strategy is the "most biased variable" rule: At each time, choose a variable $x \in V_{t}$ that maximizes the "bias" $\left|\mu_{x}^{[\omega]}\left(\Phi_{t}\right)-\frac{1}{2}\right|$ and assign it randomly based on the BP estimate. Experimentally, the most biased variable rule allows for slightly better results than the vanilla rule. For instance, in random 4-SAT, experiments indicate that the former succeeds up to $m / n=9.24$ and the latter up to $m / n=9.05$ [Selman et al. 2009; Semerjian and Ricci-Tersenghi 2009].

To my knowledge, the statistical mechanics ideas that underpin Belief Propagationguided decimation do not provide a convincing quantitative endorsement for a preference for the "most biased variable" rule over the "vanilla" strategy. But one heuristic argument in favor of "most biased variable" is that it might reduce the effect of numerical errors building up [Semerjian et al. 2007b]. The present analysis does not seem to extend to the "most biased variable" in a straightforward manner. Thus, analyzing it remains an interesting open problem. So does analyzing more complicated messagepassing-based algorithms, such as ones that involve backtracking [Ricci-Tersenghi et al. 2015].

1.3.2. Comparison with Combinatorial Algorithms. The difference between the previously studied combinatorial algorithms for random $k$-SAT and Belief Propagation can be explained nicely in terms of the factor graph. Indeed, to decide on the value of a variable, the previous algorithms only took the clauses and variables at distance two [Achlioptas and Sorkin 2000; Upfal et al. 1993; Franco and Chao 1990; Mick 1992; Suen and Frieze 1996; Sudan and Gamarnik 2003; Lalas et al. 2006] or four [Coja-Oghlan 2010] into consideration. Based on this information, the variable is assigned following some simple combinatorial rule.

BPdec can be viewed as a systematic way of making a "less shortsighted" decision. The algorithm takes into account clauses/variables at distance up to $2 \omega$, where $\omega$ may be a function that grows with $n$. Indeed, the idea of determining the marginal $M_{x_{t+1}}^{[\omega]}\left(\boldsymbol{\Phi}_{t}\right)$ yields a meaningful way of incorporating the data from all these clauses/variables. In particular, BPdec implicitly implements many of the rules that are used in the combinatorial algorithms (e.g., the "Unit Clause" rule). In this sense, BPdec can be seen as a clever generalization of many of these combinatorial algorithms. However, this also means that the techniques used in the previous analyses of combinatorial algorithms are insufficient to tackle BPdec.

\subsection{The Statistical Physics Perspective}

1.4.1. Clustering and Correlation Decay. Closely following the nonrigorous paper [RicciTersenghi et al. 2007], we discuss in this section the statistical mechanics motivation for BPdec. This will provide the basis for the discussion of the nonrigorous predictions of the algorithm's performance.

According to the physicists' "cavity method," the random formula $\Phi$ undergoes several further phase transitions prior to the satisfiability threshold. These phase transitions affect the correlations between the truth values that can be assigned to different variables. Thus, fix a variable $x$ and let $\omega=\omega(n)=o(\ln n)$ be a function that tends to infinity slowly, say $\omega=\lceil\ln \ln n\rceil$. Furthermore, let $\mathcal{B}$ be the set of all variables at distance exactly $2 \omega$ from $x$ in the factor graph. How do the values assigned to variables on the "far away boundary" $\mathcal{B}$ affect the truth value of $x$ ?

The strongest possible decay of correlations occurs when the boundary $\mathcal{B}$ has no impact on $x$ at all. To formalize this, let $\tau: \rightarrow\{-1,1\}$ be a satisfying assignment of $\Phi$ 
and let $M_{x}^{[\omega]}(\boldsymbol{\Phi}, \tau)$ be the fraction of all satisfying assignments of $\boldsymbol{\Phi}$ that set $x$ to true and that coincide with $\tau$ on $\mathcal{B}$. In symbols,

$$
M_{x}^{[\omega]}(\boldsymbol{\Phi}, \tau)=\frac{\mid\{\sigma \in \mathcal{S}(\boldsymbol{\Phi}): \sigma(x)=1, \sigma(y)=\tau(y) \text { for all } y \in \mathcal{B}\} \mid}{\mid\{\sigma \in \mathcal{S}(\boldsymbol{\Phi}): \sigma(y)=\tau(y) \text { for all } y \in \mathcal{B}\} \mid} .
$$

Also recall that $M_{x}(\boldsymbol{\Phi})$ denotes the marginal probability that $x$ takes the value "true" in a random satisfying assignment of $\boldsymbol{\Phi}$ (without any boundary condition). The Gibbs uniqueness condition requires that

$$
\max _{\tau \in \mathcal{S}(\boldsymbol{\Phi})}\left|M_{x}^{[\omega]}(\boldsymbol{\Phi}, \tau)-M_{x}(\boldsymbol{\Phi})\right|=o(1) .
$$

In other words, fixing the "far away" variables does not make it noticeably more or less likely for $x$ to take the value "true.'. Hence, the marginal $M_{x}(\Phi)$ is governed entirely by the effects of variables at distance less than $2 \omega$ from $x$ (i.e., by the local structure of the formula).

Consequently, it seems reasonable to expect that Belief Propagation yields the correct marginals so long as Equation (6) holds. It is known rigorously that with high probability, Equation (6) holds up to $r \sim r_{u}=2 \ln k / k$ (a function that tends to zero for large $k$ ) and that Belief Propagation does indeed yield the correct marginals for such densities [Shah and Montanari 2007a]. That is, for $r<r_{u}$ with high probability:

$$
\left|\mu_{x}^{[\omega]}(\boldsymbol{\Phi})-M_{x}(\boldsymbol{\Phi})\right|=o(1) \quad \text { for any } x \in V \text {. }
$$

To define the second correlation decay property, let us denote by $\tau$ a uniformly random element of $\mathcal{S}(\boldsymbol{\Phi})$. Then, the nonreconstruction condition is that

$$
\mathrm{E}_{\tau}\left|M_{x}^{[\omega]}(\boldsymbol{\Phi}, \boldsymbol{\tau})-M_{x}(\boldsymbol{\Phi})\right|=o(1) .
$$

Hence, fixing the far away boundary to a "typical" satisfying assignment has no discernible effect on $x$. Neglecting a $o(1)$-fraction of "atypical" cases $\tau$, one might still expect Equation (7) to hold so long as Equation (8) is satisfied. However, this conjecture awaits a rigorous proof. According to the cavity method, Equation (8) holds up to $r \sim r_{d}=2^{k} \ln (k) / k$. Moreover, the best rigorously analyzed algorithm (which is based on local search) succeeds in finding a satisfying assignment in polynomial time right up to $r \sim r_{d}$ with high probability [Coja-Oghlan 2010].

To state the third property, let us denote the joint distribution of the truth values of the variables $\mathcal{B}$ under a random satisfying assignment by $M_{\mathcal{B}}$. Thus, $M_{\mathcal{B}}$ is a probability distribution over $\{-1,1\}^{\mathcal{B}}$. Then, the replica symmetry condition requires that the truth values of the variables in $\mathcal{B}$ are asymptotically independent. Formally,

$$
\left|M_{\mathcal{B}}(\tau)-\prod_{x \in \mathcal{B}: \tau(x)=1} M_{x}(\boldsymbol{\Phi}) \cdot \prod_{x \in \mathcal{B}: \tau(x)=-1}\left(1-M_{x}(\boldsymbol{\Phi})\right)\right|=o(1) \quad \text { for any } \tau \in\{-1,1\}^{\mathcal{B}} .
$$

It has duly been conjectured in Ricci-Tersenghi et al. [2007] that Equation (9) suffices to obtain Equation (7); that is, to ensure that Belief Propagation yields the correct marginals on $\boldsymbol{\Phi}$. The cavity method predicts that Equation (9) holds for

$$
r \leq r_{c}=2^{k} \ln 2-3 \ln 2+O\left(2^{-k}\right),
$$

while the conjectured satisfiability threshold [Zecchina et al. 2006] is

$$
r_{k-\mathrm{SAT}}=2^{k} \ln 2-\frac{1+\ln 2}{2}+O\left(2^{-k}\right) \approx r_{c}+0.19
$$

The densities $r_{d}, r_{c}$ are also conjectured to mark a change in the geometry of the set $\mathcal{S}(\boldsymbol{\Phi})$ of satisfying assignments. Let us turn $\mathcal{S}(\boldsymbol{\Phi})$ into a graph by considering $\sigma, \tau \in \mathcal{S}(\boldsymbol{\Phi})$ 
adjacent if their Hamming distance is equal to 1 . While for densities $r<r_{d}$ the graph $\mathcal{S}(\boldsymbol{\Phi})$ is conjectured to be (essentially) connected, for $r_{d}<r<r_{c}$ it shatters into an exponential number of tiny connected components with high probability. More precisely, $\mathcal{S}(\boldsymbol{\Phi})$ admits a decomposition

$$
\mathcal{S}(\boldsymbol{\Phi})=\bigcup_{i=1}^{N} \mathcal{C}_{i}
$$

into "clusters" $\mathcal{C}_{i}$ such that $\left|\mathcal{C}_{i}\right| \leq \exp (-\Omega(n))|\mathcal{S}(\boldsymbol{\Phi})|$ for all $i$ and such that any two satisfying assignments in different clusters have Hamming distance $\Omega(n)$. This decomposition was established rigorously in Coja-Oghlan and Achlioptas [2008]. Intuitively, the cluster decomposition explains why Equation (8) fails to hold for $r>r_{d}$ : The conditional marginal $M_{x}^{[\omega]}(\boldsymbol{\Phi}, \tau)$ corresponds to the marginal of $x$ within the cluster of $\tau$, in contrast to the marginal $M_{x}(\boldsymbol{\Phi})$ over the entire set of satisfying assignments.

Furthermore, for $r_{c}<r<r_{k-\mathrm{SAT}}$ the set of satisfying assignments still decomposes into exponentially many well-separated clusters with high probability. But now a bounded number of clusters are conjectured to dominate. That is, if we order the clusters by size $\left|\mathcal{C}_{1}\right| \geq \cdots \geq\left|\mathcal{C}_{N}\right|$, then for a bounded number $1<\gamma=O(1)$ we have

$$
|\mathcal{S}(\boldsymbol{\Phi})| \sim\left|\mathcal{C}_{1} \cup \cdots \cup \mathcal{C}_{\gamma}\right| \text {. }
$$

This structure goes by the name of condensation in physics. The values that different variables take within each cluster $\mathcal{C}_{1}, \ldots, \mathcal{C}_{\gamma}$ are conjectured to be heavily dependent. Furthermore, Equation (12) implies that $M_{\mathcal{B}}$ is but a convex combination of a small (bounded) number of such intra-cluster distributions. Hence, the "condensed" geometry of Equation (12) appears to be irreconcilable with the factorization property of Equation (9).

1.4.2. Belief Propagation. Based on this "static" picture, three different hypotheses have been put forward on the likely performance of Belief Propagation-guided decimation. Most optimistically, the authors of Ricci-Tersenghi et al. [2007] argue that Belief Propagation Guided Decimation ought to find satisfying assignments efficiently for densities right up to $r_{c}$. Their prediction derives from the opinion that Equation (9) should be sufficient to obtain Equation (7), and that Equation (7) is the key to the success of Belief Propagation Guided Decimation. Specifically, Ricci-Tersenghi et al. [2007] refer to the "most biased variable" variant. However, the precise decimation strategy is irrelevant to their considerations, which are in effect at odds with Theorem 1.1.

A second prediction is that Belief Propagation Guided Decimation should fail to find satisfying assignments for $r>r_{d}$ [Braunstein et al. 2005]. This conjecture is based on the hunch that the decomposition of $\mathcal{S}(\boldsymbol{\Phi})$ into "clusters" and the ensuing demise of Equation (8) cause Equation (7) to fail. Agreeing with Ricci-Tersenghi et al. [2007], the authors appear to view Equation (7) as the key to the performance of BPdec.

According to the third prediction [Semerjian and Ricci-Tersenghi 2009], BPdec fails for densities $r>\rho_{0} \cdot 2^{k} / k$, with $\rho_{0}>0$ an absolute constant (independent of $k$ ). This prediction is based on a nonrigorous analysis of the decimation process; that is, the idealized thought experiment that BPdec strives to implement (Experiment 1.2). Crucially, the authors of Semerjian and Ricci-Tersenghi [2009] realize that Equation (7) does not guarantee the success of BPdec.

Instead, their analysis indicates that as the decimation process proceeds to assign variables, the remaining unassigned variables are bound by clauses that become shorter and shorter. In effect, the clauses become increasingly difficult to satisfy, and thus the remaining set of satisfying assignments shrinks rapidly. In other words, successive decimation of variables has a similar effect as increasing the density of the formula. Consequently, after a number $t$ of decimations, BPdec may wind up with a 
formula $\boldsymbol{\Phi}_{t}$ that violates Equation (9) and thus Equation (7), even though the initial formula $\boldsymbol{\Phi}$ may well have satisfied those conditions. The contribution [Semerjian and Ricci-Tersenghi 2009] supersedes an earlier attempt at studying the effect of decimation [Semerjian et al. 2007b].

Theorem 1.1 is in agreement with the prediction from Semerjian and Ricci-Tersenghi [2009]. But an important advantage of the present work over the (nonrigorous) contribution [Semerjian and Ricci-Tersenghi 2009] is that here we manage to analyze the actual algorithm BPdec. By contrast, Semerjian and Ricci-Tersenghi [2009] only deals with the decimation process (i.e., Experiment 1.2, the idealized experiment that assumes knowledge of the precise marginals). That is, going significantly beyond the ambition of Semerjian and Ricci-Tersenghi [2009], here we develop a technique for explicitly analyzing the dynamics of the message passing procedure.

In summary, the predictions in Braunstein et al. [2005] and Ricci-Tersenghi et al. [2007] as to the performance of Belief Propagation are inaccurate because they ignore the effect of decimation. By contrast, as conjectured in Semerjian and Ricci-Tersenghi [2009] and proved here, in actuality BPdec gets itself into trouble by assigning and decimating one variable after the other. Thus, computing the correct marginals in the original formula $\Phi$ is one thing, but continuing to do so as decimation proceeds is quite another. ${ }^{2}$

1.4.3. Survey Propagation. Let us briefly comment on Survey Propagation Guided Decimation, the physicists' flagship algorithm [Braunstein et al. 2005; Zecchina et al. 2002]. It is based on the idea of working with a different probability distribution. Namely, instead of the uniform probability over satisfying assignments, Survey Propagation aims for the uniform distribution over the clusters $\mathcal{C}_{i}$ in the decomposition of Equation (11). These clusters can be encoded as generalized assignments $\tau: V \rightarrow\{-1,0,1\}$, with $\tau(x)= \pm 1$ indicating that variable $x$ takes the value \pm 1 in all the assignments in $\mathcal{C}_{i}$, and $\tau(x)=0$ indicating that $x$ can take either value [Wainwright et al. 2007; Mézard and Montanari 2009]. Survey Propagation Guided Decimation combines a messagepassing algorithm for approximating the marginals of these generalized assignments with a decimation procedure (see Braunstein et al. [2005] for details).

According to the cavity method, the Survey Propagation distribution enjoys a factorization property akin to Equation (9) for densities $r$ right up to $r_{k-S A T}$. In fact, the physicists' computation of the conjectured $r_{k-S A T}$ depends on this assumption [Zecchina et al. 2006; Mézard and Montanari 2009]. Furthermore, the (conjectured) factorization property nurtured hopes that Survey Propagation may perform well for densities "close to" $r_{k-\mathrm{SAT}}$ [Braunstein et al. 2005; Ricci-Tersenghi et al. 2007; Zecchina et al. 2002].

Given the preceding discussion of Belief Propagation, the obvious problem with this forecast is that it ignores the effect of decimation. More precisely, one might be apt to think that in the undecimated random formula $\Phi$ the Survey Propagation distribution factorizes for densities right up to $r_{k-\mathrm{SAT}}$, but that this might not be the case in a formula $\boldsymbol{\Phi}_{t}$ where some variables have been decimated. However, the arguments of Semerjian and Ricci-Tersenghi [2009] do not seem to extend to Survey Propagation. Yet, building upon ideas from the present work, Hetterich [2016] recently analyzed a basic version of Survey Propagation Guided Decimation. The main result is that the algorithm fails to find satisfying assignments with high probability for densities $r>\left(1+\varepsilon_{k}\right) 2^{k} \ln k / k$, where $\varepsilon_{k} \rightarrow 0$ in the limit of large $k$.

\footnotetext{
${ }^{2}$ We note, as a cautionary tale, that both Ricci-Tersenghi et al. [2007] and Semerjian and Ricci-Tersenghi [2009] quote experimental evidence to support their claims. This illustrates the difficulty of producing reliable experimental results on large random CSPs and thus the need for rigorous results.
} 


\subsection{Further Related Work}

In full generality, Belief Propagation is a generic technique for computing the marginals of a probability distribution described by an "acyclic graphical model" [Pearl 1988]. But special instantiations of Belief Propagation have been (re)discovered several times for several applications. Examples include statistical inference [Pearl 1988], coding theory [Gallager 1963], and statistical mechanics [Bethe 1935], where the method is also referred to as "Bethe-Peierls approximation." For a coherent discussion, see Mézard and Montanari [2009] and the references therein.

In spite of BP's practical success (and popularity), rigorous analyses of the algorithm are scarce. A few exist in the context of LDPC decoding (e.g., Urbanke and Montanari [2007c] and Urbanke et al. [2001]). We also analyzed BP for graph 3-coloring [Vilenchik et al. 2009] on a certain class of expander graphs. A further related result deals with the conceptually much simpler Warning Propagation algorithm on certain random 3CNFs ("planted model") [Vilenchik et al. 2013]. In the random $k$-XORSAT problem (random linear equations mod 2), Belief Propagation reduces to Warning Propagation due to the algebraic nature of the problem and can thus be analyzed easily [Mézard and Montanari 2009]. Furthermore, there has been some recent progress on analyzing certain variants of BP (such as the "max-product algorithm") for certain optimization problems that are polynomial-time solvable in the worst case (e.g., Bayati et al. [2008] and Wei et al. [2012]).

A distinctive feature of Belief Propagation Guided Decimation in comparison to earlier algorithmic applications of Belief Propagation is the decimation step (that the algorithm assigns one variable at a time and reruns Belief Propagation on the reduced formula). In terms of analyzing the algorithm, decimation poses a substantial challenge. The present article furnishes the first analysis of this kind of algorithm on a nontrivial type of instances. By contrast, previous analyses deal with algorithms that use BP in a "one shot" fashion; that is, the supposed marginals obtained via BP are used directly to assign all variables at once [Vilenchik et al. 2009; Urbanke and Montanari 2007c; Urbanke et al. 2001]. Roughly speaking, this approach seems to work best if the problem instances are somewhat overconstrained so that there is (essentially) a unique solution. By contrast, as we saw in Section 1.4 for $r<r_{k-S A T}$, the random formula $\Phi$ with high probability has exponentially many satisfying assignments whose typical pairwise distance is close to $\frac{n}{2}$ with high probability. Furthermore, as we saw in Section 1.4, it is the decimation step that precipitates the demise of Belief Propagation Guided Decimation.

In the context of random constraint satisfaction problems, Belief Propagation works out to be a special (the "replica symmetric") case of a larger statistical mechanics framework called the cavity method [Mézard and Montanari 2009]. The cavity method provides a toolbox for deriving highly nontrivial exact conjectures on various phase transitions in random CSPs. The conjectured value in Equation (10) of the $r_{k-S A T}$ is an example, but the method is quite general and has been applied to a host of further CSPs as well.

The study of the BP marginals on the undecimated random formula $\Phi$ is somewhat related to the so-called reconstruction problem. This problem has been studied on "symmetric" random CSPs, which include problems such as (hyper)graph coloring [Tetali et al. 2011], but not $k$-SAT. The proofs in Tetali et al. [2011] are based on indirect arguments (related to the second moment method), which do not seem to extend to an analysis of BPdec.

Recently, Sudan and Gamarnik [2014] pointed out an interesting connection between the demise of "local" algorithms and the geometry of the set of solutions of the random $k$-NAESAT problem. This analysis has some implications on the performance of Belief/ Survey Propagation Guided Decimation for the random $k$-NAESAT, albeit only in the case that, in each step, message-passing is run merely for a bounded number of 
iterations. This is arguably unsatisfactory (see the discussion in Section 1.3). The present article allows for an unbounded number of iterations (see Remark 1.5). Additionally, the constraint density $r$ required by Sudan and Gamarnik [2014] is substantially higher than the lower bound in Theorem 1.1. Yet an interesting achievement of Sudan and Gamarnik [2014] is to link the performance of a reasonable class of algorithms to the geometry of the set of NAE solutions.

\subsection{Preliminaries and Notation}

In this section, we collect a few well-known results and introduce a bit of notation. First, we note for later reference a well-known estimate of the expected number of satisfying assignments (see, e.g., Achlioptas and Peres [2004] for a derivation).

Lemma 1.7. We have $\mathrm{E}|\mathcal{S}(\boldsymbol{\Phi})|=\Theta\left(2^{n}\left(1-2^{-k}\right)^{m}\right) \leq 2^{n} \exp \left(-r n / 2^{k}\right)$.

Furthermore, we are going to need the following Chernoff bound on the tails of a binomially distributed random variable or, more generally, a sum of independent Bernoulli trials [Ruciński et al. 2000, p. 21]:

Lemma 1.8. Let $X$ be a sum of independent Bernoulli variables with mean $\mu>0$. Let

$$
\varphi(x)=(1+x) \ln (1+x)-x .
$$

Then for any $t>0$,

$$
\mathrm{P}[X>\mu+t] \leq \exp (-\mu \cdot \varphi(t / \mu)), \quad \mathrm{P}[X<\mu-t] \leq \exp (-\mu \cdot \varphi(-t / \mu)) .
$$

In particular, for any $t>1$ we have $\mathrm{P}[X>t \mu] \leq \exp [-t \mu \ln (t / \mathrm{e})]$.

For a real $b \times a$ matrix $\Lambda$ let

$$
\|\Lambda\|_{\square}=\max _{\zeta \in \mathbf{R}^{a} \backslash\{0\}} \frac{\|\Lambda \zeta\|_{1}}{\|\zeta\|_{\infty}} .
$$

Thus, $\|\Lambda\|_{\square}$ is the norm of $\Lambda$ viewed as an operator from $\mathbf{R}^{a}$ equipped with the $L^{\infty}$ norm to $\mathbf{R}^{b}$ endowed with the $L^{1}$-norm. For a set $A \subset[a]=\{1, \ldots, a\}$, we let $\mathbf{1}_{A} \in\{0,1\}^{a}$ denote the indicator vector of $A$. The following well-known fact about the norm $\|\cdot\|_{\square}$ of matrices with diagonal entries equal to zero is going to come in handy:

FACT 1.9. For a real $b \times$ a matrix $\Lambda$ with zeros on the diagonal we have

$$
\|\Lambda\|_{\square} \leq 24 \max _{A \subset[a], B \subset[b]: A \cap B=\emptyset}\left|\left\langle\Lambda \mathbf{1}_{A}, \mathbf{1}_{B}\right\rangle\right| .
$$

Finally, throughout this article, we let $S_{n}$ denote the set of permutations of $[n]$.

\section{THE PROBABILISTIC FRAMEWORK FOR ANALYZING BPDEC}

\subsection{Outline}

The single most important technique for analyzing algorithms on the random input $\Phi$ is the "method of deferred decisions." Where it applies, the dynamics of the algorithm can typically be traced tightly via differential equations, martingales, or Markov chains. Virtually all previous analyses of algorithms for random $k$-SAT are based on this approach [Achlioptas and Sorkin 2000; Upfal et al. 1993; Franco and Chao 1990; Mick 1992; Coja-Oghlan 2010; Suen and Frieze 1996; Sudan and Gamarnik 2003; Lalas et al. 2006]. Unfortunately, the "deferred decisions" technique is limited to very simple, "shortsighted" algorithms that decide the value of a variable $x$ on the basis of the clauses/variables at distance; say, one or two from $x$ in the factor graph [Achlioptas and Sorkin 2000]. By contrast, in order to assign some variable $x_{t}$, BPdec explores clauses at distance up to $2 \omega$ from $x_{t}$, where (potentially) $\omega=\omega(n) \rightarrow \infty$. This renders a "deferred decisions" approach hopeless. 
Therefore, to prove Theorem 1.1, we need a fundamentally different strategy. In this section, we set up the probabilistic framework for the analysis. We will basically reduce the analysis of BPdec to the problem of analyzing the BP operator on the formula that is obtained from $\Phi$ by substituting "true" for the first $t$ variables $x_{1}, \ldots, x_{t}$ and simplifying (Theorem 2.5). In the next section, we will show that this decimated formula enjoys a few simple quasi-randomness properties with probability extremely close to one. Finally, we will show that these properties suffice to trace the BP computation.

Applied to a fixed, nonrandom formula $\Phi$ on $V=\left\{x_{1}, \ldots, x_{n}\right\}$, BPdec yields an assignment $\sigma: V \rightarrow\{-1,1\}$ (that may or may not be satisfying). This assignment is random because BPdec itself is randomized. Hence, for any fixed $\Phi$, running BPdec $(\Phi)$ induces a probability distribution $\beta_{\Phi}$ on $\{-1,1\}^{V}$. With $\mathcal{S}(\Phi)$ the set of all satisfying assignments of $\Phi$, the "success probability" of BPdec on $\Phi$ is just

$$
\operatorname{success}(\Phi)=\beta_{\Phi}(\mathcal{S}(\Phi)) \text {. }
$$

Thus, to establish Theorem 1.1, we need to show that in the random formula,

$$
\operatorname{success}(\boldsymbol{\Phi})=\beta_{\boldsymbol{\Phi}}(\mathcal{S}(\boldsymbol{\Phi}))=\exp (-\Omega(n))
$$

is exponentially small with high probability. To this end, we are going to prove that the measure $\beta_{\Phi}$ is rather close to the uniform distribution on $\{-1,1\}^{V}$ with high probability, of which $\mathcal{S}(\boldsymbol{\Phi})$ constitutes only an exponentially small fraction.

To facilitate the analysis, we are going to work with a slightly modified version of BPdec. While the original BPdec assigns the variables in the natural order $x_{1}, \ldots, x_{n}$, the modified version PermBPdec chooses a permutation $\pi$ of $[n]$ uniformly at random and assigns the variables in the order $x_{\pi(1)}, \ldots, x_{\pi(n)}$. Let $\bar{\beta}_{\Phi}$ denote the probability distribution induced on $\{-1,1\}^{V}$ by $\operatorname{PermBPdec}(\Phi)$. Because the uniform distribution over $k$-CNFs is invariant under permutations of the variables, we obtain

FACT 2.1. If $\bar{\beta}_{\boldsymbol{\Phi}}(\mathcal{S}(\boldsymbol{\Phi})) \leq \exp (-\Omega(n))$ with high probability, then success $(\boldsymbol{\Phi})=$ $\beta_{\Phi}(\mathcal{S}(\Phi)) \leq \exp (-\Omega(n))$ with high probability.

Let $\Phi$ be a $k$-CNF. Given a permutation $\pi$ and a partial assignment $\sigma:\left\{x_{\pi(s)}\right.$ : $s \leq t\} \rightarrow\{-1,1\}$, we let $\Phi_{t, \pi, \sigma}$ denote the formula obtained from $\Phi$ by substituting the values $\sigma\left(x_{\pi(s)}\right)$ for the variables $x_{\pi(s)}$ for $1 \leq s \leq t$ and simplifying. Formally, $\Phi_{t, \pi, \sigma}$ is obtained from $\Phi$ as follows:

- Remove all clauses $a$ of $\Phi$ that contain a variable $x_{\pi(s)}$ with $1 \leq s \leq t$ such that $\sigma\left(x_{\pi(s)}\right)=\operatorname{sign}\left(x_{\pi(s)}, a\right)$.

-For all clauses $a$ that contain a $x_{\pi(s)}$ with $1 \leq s \leq t$ such that $\sigma\left(x_{\pi(s)}\right) \neq \operatorname{sign}\left(x_{\pi(s)}, a\right)$, remove $x_{\pi(s)}$ from $a$.

- Remove any empty clauses (resulting from clauses of $\Phi$ that become unsatisfied if we set $x_{\pi(s)}$ to $\sigma\left(x_{\pi(s)}\right)$ for $\left.1 \leq s \leq t\right)$ from the formula.

For a number $\delta>0$ and an index $l>t$, we say that $x_{\pi(l)}$ is $(\delta, t)$-biased if

$$
\left|\mu_{x_{\pi(l)}^{[\omega]}}^{[\omega}\left(\Phi_{t, \pi, \sigma}\right)-1 / 2\right|>\delta .
$$

Moreover, the triple $(\Phi, \pi, \sigma)$ is $(\delta, t)$-balanced if no more than $\delta(n-t)$ variables are $(\delta, t)$-biased.

Let $\pi$ be the permutation chosen by $\operatorname{PermBPdec}(\Phi)$, and let $\sigma$ be the partial assignment constructed in the first $t$ steps. The variable $x_{\pi(t+1)}$ is uniformly distributed over the set $V \backslash\left\{x_{\pi(s)}: s \leq t\right\}$ of currently unassigned variables. Hence, if $(\Phi, \pi, \sigma)$ is $(\delta, t)$-balanced, then the probability that $x_{\pi(t+1)}$ is $(\delta, t)$-biased is bounded by $\delta$. (This conclusion was the purpose of decimating the variables in a random order.) Furthermore, given that 
$x_{\pi(t+1)}$ is not $(\delta, t)$-biased, the probability that PermBPdec will set it to true lies in the interval $\left[\frac{1}{2}-\delta, \frac{1}{2}+\delta\right]$. Consequently,

$$
\mid \frac{1}{2}-\mathrm{P}\left[\sigma\left(x_{\pi(t+1)}\right)=1 \mid(\Phi, \pi, \sigma) \text { is }(\delta, t) \text {-balanced }\right] \mid \leq 2 \delta .
$$

Thus, the smaller $\delta$, the closer $\sigma\left(x_{\pi(t+1)}\right)$ comes to being uniformly distributed. Hence, if $(\delta, t)$-balancedness holds for all $t$ with a "small" $\delta$, then $\bar{\beta}_{\Phi}$ will be close to the uniform distribution on $\{-1,1\}^{V}$.

To put this observation to work, we define

$$
\delta_{t}=\exp (-c(1-t / n) k) \quad \text { and } \quad \hat{t}=\left(1-\frac{\ln \left(k r / 2^{k}\right)}{c^{2} k}\right) n
$$

where $c>0$ is a small enough absolute constant. ${ }^{3}$ In addition, we let

$$
\Delta_{t}=\sum_{s=1}^{t} \delta_{t}
$$

Lemma 2.2. For any $0 \leq t \leq \hat{t}$ we have

$$
\Delta_{t} \leq(1+o(1)) \frac{n}{c k \exp (c k(1-t / n))}=(1+o(1)) \frac{n \delta_{t}}{c k} .
$$

Furthermore, $\Delta_{\hat{t}} \sim \frac{n}{c k}\left[\left(k r / 2^{k}\right)^{-1 / c}-\exp (-c k)\right]$.

Proof. We have

$$
\Delta_{t}=\sum_{s=1}^{t} \delta_{s}=\exp (-c k) \sum_{s=1}^{t} \exp (c s k / n)=\exp (-c k)\left[\frac{\exp (c k(t+1) / n)-1}{\exp (c k / n)-1}-1\right] .
$$

Since $\exp (c k / n)=1+c k / n+O\left(n^{-2}\right)$ and $\hat{t}=\Omega(n)$, we obtain from Equation (16)

$$
\Delta_{\hat{t}} \sim \frac{n}{c k}\left[\exp \left(c k\left(\frac{\hat{t}}{n}-1\right)\right)-\exp (-c k)\right]=\frac{n}{c k}\left[\left(k r / 2^{k}\right)^{-1 / c}-\exp (-c k)\right] .
$$

Furthermore, for $1 \leq t \leq \hat{t}$, Equation (16) yields the upper bound

$$
\begin{aligned}
\Delta_{t} & \leq \exp (-c k) \cdot \frac{\exp (c k(t+1) / n)}{\exp (c k / n)-1}=\frac{\exp (c k(t-n) / n)}{1-\exp (-c k / n)} \\
& \sim \frac{n}{c k} \exp (-c k(1-t / n)),
\end{aligned}
$$

as $\exp (-c k / n)=1-c k / n+O\left(n^{-2}\right)$.

For $\xi>0$, we say that $\Phi$ is $(t, \xi)$-uniform if

$$
\mid\left\{(\pi, \sigma) \in S_{n} \times\{-1,1\}^{V}:(\Phi, \pi, \sigma) \text { is } \operatorname{not}\left(\delta_{t}, t\right) \text {-balanced }\right\} \mid \leq 2^{n} n ! \cdot \exp \left[-10\left(\xi n+\Delta_{t}\right)\right] .
$$

Proceeding by induction on $t$, we are going to use Equation (13) to relate the distribution $\bar{\beta}_{\Phi}$ to the uniform distribution on $\{-1,1\}^{V}$ for $(t, \xi)$-uniform formulas. More precisely, in Section 2.2 we are going to prove

\footnotetext{
${ }^{3}$ Setting $c=10^{-10^{10}}$ will evidently suffice, but no attempt at finding the optimal $c$ has been made.
} 
Proposition 2.3. Suppose that $\Phi$ is $(t, \xi)$-uniform for all $0 \leq t \leq \hat{t}$. Then,

$$
\bar{\beta}_{\Phi}(\mathcal{E}) \leq \frac{|\mathcal{E}|}{2^{\hat{t}}} \cdot \exp \left[6\left(\Delta_{\hat{t}}+\xi n\right)\right]+\exp (-\xi n / 2) \text { for any } \mathcal{E} \subset\{-1,1\}^{V} .
$$

Proposition 2.3 reduces the proof of Theorem 1.1 to showing that $\Phi$ is $(t, \xi)$-uniform with some appropriate probability.

To prove this, we need two simple definitions. We call a clause $a$ of a formula $\Phi$ redundant if $\Phi$ has another clause $b$ such that $a, b$ have at least two variables in common. Furthermore, we call the formula $\Phi$ tame if

i. $\Phi$ has no more than $\ln n$ redundant clauses, and

ii. no more than $\ln n$ variables occur in more than $\ln n$ clauses of $\Phi$.

The following is a well-known fact.

LeMma 2.4. The random formula $\boldsymbol{\Phi}$ is tame with high probability.

We observe that it suffices to state Lemma 2.4 as a "with high probability" statement. This is because Theorem 1.1 asserts that with high probability the random formula is such that the success probability of BPdec is exponentially small, but not that this statement holds with probability $1-\exp (-\Omega(n))$ over the choice of the random formula.

Now, the following result provides the key estimate for proving that $\Phi$ is $(t, \xi)$-uniform with a very high probability.

THEOREM 2.5. There is a constant $\rho_{0}>0$ such that, for any $k, r$ satisfying $2^{k} \rho_{0} / k \leq$ $r \leq 2^{k} \ln 2$ there is $0<\xi=\xi(k, r)<1 / k$ so that for $n$ large enough the following holds. Fix any permutation $\pi$ of $[n]$ and any assignment $\sigma \in\{-1,1\}^{V}$. Then, for any $0 \leq t \leq \hat{t}$ we have

$$
\mathrm{P}\left[(\boldsymbol{\Phi}, \pi, \sigma) \text { is }\left(\delta_{t}, t\right) \text {-balanced } \mid \boldsymbol{\Phi} \text { is tame }\right] \geq 1-\exp \left[-3 \xi n-10 \Delta_{t}\right] .
$$

We defer the proof of Theorem 2.5 to Section 3 .

COROLlaRY 2.6. In the notation of Theorem 2.5,

$$
\mathrm{P}[\forall t \leq \hat{t}: \boldsymbol{\Phi} \text { is }(t, \xi) \text {-uniform } \mid \boldsymbol{\Phi} \text { is tame }] \geq 1-\exp (-\xi n) .
$$

Proof. For $1 \leq t \leq \hat{t}$ and a $k$-CNF $\Phi$ we let $X_{t}(\Phi)$ signify the number of pairs $(\pi, \sigma) \in S_{n} \times\{-1,1\}^{V}$ such that $(\Phi, \pi, \sigma)$ fails to be $\left(\delta_{t}, t\right)$-balanced. Then, Theorem 2.5 yields

$$
\mathrm{E}\left[X_{t}(\boldsymbol{\Phi}) \mid \boldsymbol{\Phi} \text { is tame }\right] \leq 2^{n} n ! \cdot \exp \left(-3 \xi n-10 \Delta_{t}\right) .
$$

Hence, by Markov's inequality and the union bound

$$
\mathrm{P}\left[\exists t \leq \hat{t}: X_{t}(\boldsymbol{\Phi})>2^{n} n ! \cdot \exp \left(-\xi n-10 \Delta_{t}\right) \mid \boldsymbol{\Phi} \text { is tame }\right] \leq n \exp (-2 \xi n) \leq \exp (-\xi n) .
$$

Since $\Phi$ is $(t, \xi)$-uniform if $X_{t}(\Phi) \leq 2^{n} n ! \cdot \exp \left(-\xi n-10 \Delta_{t}\right)$, the assertion follows from Equation (19).

Proof of Theorem 1.1. Let us keep the notation of Theorem 2.5. By Lemma 2.4, we may condition on $\boldsymbol{\Phi}$ being tame. Let $\mathcal{U}$ be the event that $\boldsymbol{\Phi}$ is $(t, \xi)$-uniform for all $1 \leq t \leq \hat{t}$. Let $\mathcal{S}$ be the event that $|\mathcal{S}(\boldsymbol{\Phi})| \leq n \cdot \mathrm{E}|\mathcal{S}(\boldsymbol{\Phi})|$. By Corollary 2.6 and Markov's inequality, we have $\Phi \in \mathcal{U} \cap \mathcal{S}$ with high probability. If $\Phi \in \mathcal{U} \cap \mathcal{S}$, then, by Proposition 2.3,

$$
\begin{aligned}
\bar{\beta}_{\boldsymbol{\Phi}}(\mathcal{S}(\boldsymbol{\Phi})) & \leq \frac{|\mathcal{S}(\boldsymbol{\Phi})|}{2^{\hat{t}}} \cdot \exp \left[6\left(\Delta_{\hat{t}}+\xi n\right)\right]+\exp (-\xi n / 2) \\
& \leq n \cdot \mathrm{E}|\mathcal{S}(\boldsymbol{\Phi})| \cdot 2^{-\hat{t}} \exp \left[6\left(\Delta_{\hat{t}}+\xi n\right)\right]+\exp (-\xi n / 2)
\end{aligned}
$$


By Lemmas 1.7 and 2.2, we have $\mathrm{E}|\mathcal{S}(\boldsymbol{\Phi})| \leq 2^{n} \exp \left(-r n / 2^{k}\right)$ and $\Delta_{\hat{t}} \leq \frac{n}{c k}\left(k r / 2^{k}\right)^{-1 / c}$. Plugging these estimates and the definition of Equation (14) of $\hat{t}$ into Equation (20), we find that given $\Phi \in \mathcal{U} \cap \mathcal{S}$,

$$
\bar{\beta}_{\boldsymbol{\Phi}}(\mathcal{S}(\boldsymbol{\Phi})) \leq n \exp \left[n\left(-\frac{r}{2^{k}}+\frac{\ln \left(k r / 2^{k}\right) \ln 2}{c^{2} k}+6 \xi+\frac{6}{c k}\left(k r / 2^{k}\right)^{-1 / c}\right)\right]+\exp (-\xi n / 2) .
$$

Recalling that $\rho=k r / 2^{k}$ and $\xi \leq 1 / k$, we thus obtain

$$
\bar{\beta}_{\boldsymbol{\Phi}}(\mathcal{S}(\boldsymbol{\Phi})) \leq n \exp \left[-\frac{n}{k}\left(\rho-\frac{\ln 2 \ln \rho}{c^{2}}-6-\frac{6}{c \rho^{1 / c}}\right)\right]+\exp (-\xi n / 2) .
$$

Hence, if $\rho \geq \rho_{0}$ for a sufficiently large constant $\rho_{0}>0$, then Equation (21) yields $\bar{\beta}_{\Phi}(\mathcal{S}(\boldsymbol{\Phi}))=\exp (-\Omega(n))$. Finally, Theorem 1.1 follows from Fact 2.1.

\subsection{Proof of Proposition 2.3}

We consider an additional variant of BPdec that receives the order $\pi$ in which variables are to be decimated as an input parameter.

Algorithm 2.7. $\operatorname{BPdec}(\Phi, \pi)$

Input: A $k$-SAT formula $\Phi$ on $V=\left\{x_{1}, \ldots, x_{n}\right\}$ and a permutation $\pi \in S_{n}$.

Output: An assignment $\sigma \rightarrow\{-1,1\}$.

0 . Let $\Phi_{0}=\Phi$.

1. For $t=0, \ldots, n-1$ do

2. Compute the BP results $\mu_{x}^{[\omega]}\left(\Phi_{t}\right)$.

3. Let

$$
\sigma\left(x_{\pi(t+1)}\right)=\left\{\begin{array}{cl}
1 & \text { with probability } \mu_{x_{\pi}(t+1)}^{[\omega]}\left(\Phi_{t}\right), \\
-1 & \text { with probability } 1-\mu_{x_{\pi(t+1)}}^{[\omega]}\left(\Phi_{t}\right) .
\end{array}\right.
$$

4. Obtain $\Phi_{t+1}$ from $\Phi_{t}$ by substituting the value $\sigma\left(x_{\pi(t+1)}\right)$ for $x_{\pi(t+1)}$ and simplifying.

5. Return the assignment $\sigma$.

Fix a $k$-CNF $\Phi$ that is $(t, \xi)$-uniform for all $1 \leq t \leq \hat{t}$. Let $S_{n}$ be the set of all permutations on $[n]$. Let $\lambda_{\Phi}$ be the probability distribution on pairs $(\pi, \sigma) \in S_{n} \times\{-1,1\}^{V}$ induced by choosing a permutation $\pi \in S_{n}$ uniformly at random and letting $\sigma=$ $\operatorname{BPdec}(\Phi, \pi)$. Then, $\bar{\beta}_{\Phi}$ is the $\pi$-marginal of $\lambda_{\Phi}$; that is,

$$
\bar{\beta}_{\Phi}(\mathcal{E})=\lambda_{\Phi}\left(S_{n} \times \mathcal{E}\right) \text { for any } \mathcal{E} \subset\{-1,1\}^{V} .
$$

To study $\lambda_{\Phi}$, we consider another distribution $\lambda_{\Phi}^{\prime}$ on pairs $\left(\pi, \sigma^{\prime}\right) \in S_{n} \times\{ \pm 1\}^{V}$ that is easier to analyze and that will turn out to be close to $\lambda_{\Phi}$. To define $\lambda_{\Phi}^{\prime}$, let $\mathcal{B}_{t}$ be the set of all pairs $(\pi, \sigma)$ such that $(\Phi, \pi, \sigma)$ is not $\left(\delta_{t}, t\right)$-balanced. Moreover, let $\mathcal{B}=\bigcup_{t=0}^{\hat{t}} \mathcal{B}_{t}$. The distribution $\lambda_{\Phi}^{\prime}$ is induced by choosing a permutation $\pi$ uniformly at random and running the following algorithm on $\Phi, \pi$ :

Algorithm 2.8. $\quad \operatorname{BPdec}^{\prime}(\Phi, \pi)$

Input: A $k$-SAT formula $\Phi$ on $V=\left\{x_{1}, \ldots, x_{n}\right\}$ and a permutation $\pi \in S_{n}$.

Output: An assignment $\sigma^{\prime}: V \rightarrow\{-1,1\}$.

0. Let $\Phi_{0}=\Phi$.

1. For $t=0, \ldots, n-1$ do

2. Compute the BP results $\mu_{x}^{[\omega]}\left(\Phi_{t}\right)$. 
3. If $\left(\Phi, \pi, \sigma^{\prime}\right)$ is $\left(\delta_{t}, t\right)$-balanced, then

let

$$
\sigma^{\prime}\left(x_{\pi(t+1)}\right)=\left\{\begin{array}{cl}
1 & \text { with probability } \mu_{x_{\pi(t+1)}^{[\omega]}}^{[\omega]}\left(\Phi_{t}\right), \\
-1 & \text { with probability } 1-\mu_{x_{\pi(t+1)}}^{[\omega]}\left(\Phi_{t}\right) .
\end{array}\right.
$$

else let $\sigma^{\prime}\left(x_{\pi(t)}\right)=\zeta$ with probability $\frac{1}{2}$ for $\zeta= \pm 1$.

4. Obtain $\Phi_{t+1}$ from $\Phi_{t}$ by substituting the value $\sigma^{\prime}\left(x_{\pi(t+1)}\right)$ for $x_{\pi(t+1)}$ and simplifying.

5. Output the assignment $\sigma^{\prime}$.

Roughly speaking, BPdec' disregards the BP outcome if it strays too far from the "flat" vector $\frac{1}{2} \mathbf{1}$. We claim that $\lambda_{\Phi}$ and $\lambda_{\Phi}^{\prime}$ are related as follows. For $\mathcal{F} \subset S_{n} \times\{-1,1\}^{V}$ let

$$
\mathcal{F}_{\hat{t}}=\left\{(\pi, \sigma) \in S_{n} \times\{ \pm 1\}^{V}: \exists\left(\pi^{*}, \sigma^{*}\right) \in \mathcal{F}: \forall 1 \leq t \leq \hat{t}: \pi^{*}(t)=\pi(t), \sigma^{*}\left(x_{\pi(t)}\right)=\sigma\left(x_{\pi(t)}\right)\right\} .
$$

Thus, $\mathcal{F}_{\hat{t}}$ is the set of all $(\pi, \sigma)$ that coincides with some $\left(\pi^{*}, \sigma^{*}\right) \in \mathcal{F}$ "up to time $\hat{t}^{\text {". In }}$ particular, $\mathcal{F} \subset \mathcal{F}_{\hat{t}}$.

Lemma 2.9. For any $\mathcal{F} \subset S_{n} \times\{-1,1\}^{V}$, we have $\lambda_{\Phi}(\mathcal{F}) \leq \lambda_{\Phi}^{\prime}\left(\mathcal{F}_{\hat{t}}\right)+\lambda_{\Phi}^{\prime}(\mathcal{B})$.

Proof. By construction, for any $(\pi, \sigma) \notin \mathcal{B}_{t}$ and any $\zeta \in\{-1,1\}$, we have

$$
\begin{aligned}
\lambda_{\Phi}\left[\sigma\left(x_{\pi(t+1)}\right)=\zeta \mid \pi=\pi \wedge\right. & \forall s \\
& \left.\leq t: \sigma\left(x_{\pi(s)}\right)=\sigma\left(x_{\pi(s)}\right)\right] \\
& =\lambda_{\Phi}^{\prime}\left[\sigma^{\prime}\left(x_{\pi(t+1)}\right)=\zeta \mid \pi=\pi \wedge \forall s \leq t: \sigma^{\prime}\left(x_{\pi(s)}\right)=\sigma\left(x_{\pi(s)}\right)\right] .
\end{aligned}
$$

Hence, Bayes' rule yields that for any pair $(\pi, \sigma) \notin \mathcal{B}$,

$$
\begin{aligned}
\lambda_{\Phi}\left[\forall t \leq \hat{t}: \pi(t)=\pi(t) \wedge \sigma\left(x_{\pi(t)}\right)\right. & \left.=\sigma\left(x_{\pi(t)}\right)\right] \\
& =\lambda_{\Phi}^{\prime}\left[\forall t \leq \hat{t}: \pi(t)=\pi(t) \wedge \sigma^{\prime}\left(x_{\pi(t)}\right)=\sigma\left(x_{\pi(t)}\right)\right] .
\end{aligned}
$$

In particular, $\lambda_{\Phi}(\mathcal{B})=\lambda_{\Phi}^{\prime}(\mathcal{B})$. Hence, for any event $\mathcal{F}$ we obtain

$$
\lambda_{\Phi}(\mathcal{F}) \leq \lambda_{\Phi}\left(\mathcal{F}_{\hat{t}}\right) \leq \lambda_{\Phi}\left(\mathcal{F}_{\hat{t}} \backslash \mathcal{B}\right)+\lambda_{\Phi}(\mathcal{B}) \stackrel{(23)}{=} \lambda_{\Phi}^{\prime}\left(\mathcal{F}_{\hat{t}} \backslash \mathcal{B}\right)+\lambda_{\Phi}^{\prime}(\mathcal{B}) \leq \lambda_{\Phi}^{\prime}\left(\mathcal{F}_{\hat{t}}\right)+\lambda_{\Phi}^{\prime}(\mathcal{B}),
$$

as desired.

Let $\lambda^{\prime \prime}$ be the uniform probability distribution on $S_{n} \times\{-1,1\}^{V}$, and let $(\boldsymbol{\pi}, \boldsymbol{u})$ denote a pair chosen from $\lambda^{\prime \prime}$. To relate $\lambda_{\Phi}^{\prime}$ and $\lambda^{\prime \prime}$, let $A_{t}(\pi, \sigma)$ be equal to 1 if $(\pi, \sigma) \notin \mathcal{B}_{t}$ and $x_{\pi(t)}$ is $\left(\delta_{t}, t\right)$-biased in $(\Phi, \pi, \sigma)$, and set $A_{t}(\pi, \sigma)=0$ otherwise. In addition, let $A(\pi, \sigma)=\sum_{t \leq \hat{t}} A_{t}(\pi, \sigma)$.

Lemma 2.10. For any pair $(\pi, \sigma) \in S_{n} \times\{-1,1\}^{V}$ we have

$$
\begin{aligned}
\lambda_{\Phi}^{\prime}[\forall t \leq \hat{t}: & \left.\pi(t)=\pi(t) \wedge \sigma^{\prime}\left(x_{\pi(t)}\right)=\sigma\left(x_{\pi(t)}\right)\right] \\
& \leq \lambda^{\prime \prime}\left[\forall t \leq \hat{t}: \pi(t)=\pi(t) \wedge \boldsymbol{u}\left(x_{\pi(t)}\right)=\sigma\left(x_{\pi(t)}\right)\right] \cdot 2^{A(\pi, \sigma)} \prod_{t \leq T} 1+2 \delta_{t} .
\end{aligned}
$$

Proof. Fix any pair $(\pi, \sigma) \in S_{n} \times\{-1,1\}^{V}$ and let $\mathcal{L}_{t}$ be the event that

$$
\pi(t)=\pi(t) \text { and } \sigma^{\prime}\left(x_{\pi(t)}\right)=\sigma\left(x_{\pi(t)}\right) .
$$

Then, for any $1 \leq t \leq \hat{t}$ we can bound the conditional probability $\lambda_{\Phi}^{\prime}\left[\mathcal{L}_{t} \mid \pi(t)=\right.$ $\left.\pi(t) \wedge \wedge_{s<t} \mathcal{L}_{s}\right]$ as follows: 
Case 1: $(\pi, \sigma) \in \mathcal{B}_{t}$. In this case, $(\Phi, \pi, \sigma)$ is not $\left(\delta_{t}, t\right)$-balanced. Therefore, Step 3 of BPdec' chooses the value $\sigma^{\prime}\left(x_{\pi(t)}\right)$ uniformly. Hence, the event $\sigma^{\prime}\left(x_{\pi(t)}\right)=\sigma\left(x_{\pi(t)}\right)$ occurs with probability $\frac{1}{2}$.

Case 2: $(\pi, \sigma) \notin \mathcal{B}_{t}$ and $A_{t}(\pi, \sigma)=0$ Since $(\Phi, \pi, \sigma)$ is $\left(\delta_{t}, t\right)$-balanced, Step 3 of BPdec $'$ uses the BP marginals $\mu_{x_{\pi(t)}}^{[\omega]}(\zeta)$ in order to assign $x_{\pi(t)}$. Because $A_{t}(\pi, \sigma)=0$, the variable $x_{\pi(t)}$ is not $\left(\delta_{t}, t\right)$-biased, whence $\mu_{x_{\pi(t)}^{[\omega]}}(\zeta) \leq \frac{1}{2}+\delta_{t}$ for both $\zeta=-1$ and $\zeta=1$. Hence, the probability that $\sigma^{\prime}\left(x_{\pi(t)}\right)=\sigma\left(x_{\pi(t)}\right)$ is bounded by $\frac{1}{2}+\delta_{t}$.

Case 3: $A_{t}(\pi, \sigma)=1$ In this case, we just use the trivial fact that the probability of the event $\sigma^{\prime}\left(x_{\pi(t)}\right)=\sigma\left(x_{\pi(t)}\right)$ is bounded by $1 \leq 2\left(\frac{1}{2}+\delta_{t}\right)$.

In any case, we obtain the bound $\lambda_{\Phi}^{\prime}\left[\mathcal{L}_{t} \mid \pi(t)=\pi(t) \wedge \bigwedge_{s<t} \mathcal{L}_{s}\right] \leq 2^{A_{t}(\pi, \sigma)}\left(\frac{1}{2}+\delta_{t}\right)$. Consequently, as $\lambda^{\prime \prime}$ is the uniform distribution, we get

$$
\frac{\lambda_{\Phi}^{\prime}\left[\mathcal{L}_{t} \mid \pi(t)=\pi(t) \wedge \bigwedge_{s<t} \mathcal{L}_{s}\right]}{\lambda^{\prime \prime}\left[\mathcal{L}_{t} \mid \pi(t)=\pi(t) \wedge \bigwedge_{s<t} \mathcal{L}_{s}\right]} \leq 2^{A_{t}(\pi, \sigma)}\left(1+2 \delta_{t}\right) .
$$

Multiplying Equation (24) up for $t \leq \hat{t}$ yields the assertion.

To put Lemma 2.10 to work, we need to estimate $A\left(\pi, \sigma^{\prime}\right)$ :

Lemma 2.11. We have $\lambda_{\Phi}^{\prime}\left[A\left(\pi, \sigma^{\prime}\right)>4\left(\Delta_{\hat{t}}+\xi n\right)\right] \leq \exp (-\xi n)$.

Proof. We are going to bound the probability that $A_{t}\left(\pi, \sigma^{\prime}\right)=1$ given the values $\pi(s), \sigma^{\prime}\left(x_{\pi(s)}\right)$ for $1 \leq s<t$.

Case 1: the event $\mathcal{B}_{t}$ occurs. Then $A_{t}=0$ by definition.

Case 2: the event $\mathcal{B}_{t}$ does not occur. In this case $(\Phi, \pi, \sigma)$ is $\left(\delta_{t}, t\right)$-balanced, which means that no more than $\delta_{t}(n-t)$ variables are biased. Since the permutation $\pi$ is chosen uniformly at random, the probability that $x_{\pi(t)}$ is $\left(\delta_{t}, t\right)$-biased is bounded by $\delta_{t}$.

Thus, in either case, the conditional probability of the event $A_{t}=1$ is bounded by $\delta_{t}$. This implies that the random variable $A\left(\pi, \sigma^{\prime}\right)=\sum_{t<\hat{t}} A_{t}\left(\pi, \sigma^{\prime}\right)$ is stochastically dominated by a sum of mutually independent Bernoulli variables with means $\delta_{1}, \ldots, \delta_{\hat{t}}$. Therefore, the assertion follows from Lemma 1.8 (the Chernoff bound).

Proof of Proposition 2.3. Combining Lemmas 2.10 and 2.11, we see that

$$
\begin{aligned}
\lambda_{\Phi}^{\prime}\left[\mathcal{F}_{\hat{t}}\right] & \leq \lambda_{\Phi}^{\prime}\left[A\left(\pi, \sigma^{\prime}\right)>4\left(\Delta_{\hat{t}}+\xi n\right)\right]+\lambda_{\Phi}^{\prime}\left[\mathcal{F}_{\hat{t}} \wedge A\left(\pi, \sigma^{\prime}\right) \leq 4\left(\Delta_{\hat{t}}+\xi n\right)\right] \\
& \leq \exp (-\xi n)+\lambda^{\prime \prime}\left[\mathcal{F}_{\hat{t}}\right] \cdot 2^{4\left(\Delta_{t}+\xi n\right)} \prod_{t \leq \hat{t}} 1+2 \delta_{t} \\
& \leq \lambda^{\prime \prime}\left[\mathcal{F}_{\hat{t}}\right] \cdot \exp \left(6 \Delta_{\hat{t}}+4 \xi n\right)+\exp (-\xi n) \quad \text { for any } \mathcal{F} \subset S_{n} \times\{-1,1\}^{V} .
\end{aligned}
$$

Our assumption that $\Phi$ is $(t, \xi)$-uniform ensures that $\lambda^{\prime \prime}\left[\mathcal{B}_{t}\right] \leq \exp \left(-10\left(\xi n+\Delta_{\hat{t}}\right)\right)$ for any $t \leq \hat{t}$. Together with Equation (25), this implies that

$$
\lambda_{\Phi}^{\prime}\left[\mathcal{B}_{t}\right] \leq \lambda^{\prime \prime}\left[\mathcal{B}_{t}\right] \exp \left(6 \Delta_{\hat{t}}+4 \xi n\right)+\exp (-\xi n) \leq 2 \exp (-\xi n) \quad \text { for any } t \leq \hat{t} .
$$

Therefore, by the union bound

$$
\lambda_{\Phi}^{\prime}[\mathcal{B}] \leq 2 \hat{t} \exp (-\xi n) \leq \exp (-0.9 \xi n) .
$$


Finally, consider any $\mathcal{E} \subset\{-1,1\}^{V}$. Let $\mathcal{F}=S_{n} \times \mathcal{E}$. Then

$$
\begin{aligned}
\bar{\beta}_{\Phi}(\mathcal{E}) & =\lambda_{\Phi}[\mathcal{F}] \\
& \leq \lambda_{\Phi}^{\prime}\left[\mathcal{F}_{\hat{t}}\right]+\lambda_{\Phi}^{\prime}[\mathcal{B}] \\
& \leq \lambda_{\Phi}^{\prime}\left[\mathcal{F}_{\hat{t}}\right]+\exp (-0.9 \xi n) \\
& \leq \lambda^{\prime \prime}\left[\mathcal{F}_{\hat{t}}\right] \exp \left(6\left(\Delta_{\hat{t}}+\xi n\right)\right)+\exp (-\xi n / 2) \\
& =\frac{\left|\mathcal{F}_{\hat{t}}\right|}{n ! 2^{n}} \cdot \exp \left(6\left(\Delta_{\hat{t}}+\xi n\right)\right)+\exp (-\xi n / 2) \\
& \leq \frac{|\mathcal{E}|}{2^{\hat{t}}} \cdot \exp \left(6\left(\Delta_{\hat{t}}+\xi n\right)\right)+\exp (-\xi n / 2)
\end{aligned}
$$

[due to Equation (22)]

[by Lemma 2.9]

[by Equation (26)]

[by Equation (25)]

[as $\lambda^{\prime \prime}$ is uniform]

[by the definition of $\mathcal{F}_{\hat{t}}$ ],

as desired.

\section{TRACING THE BELIEF PROPAGATION OPERATOR}

\subsection{Overview}

The goal in this section (and the remainder of the article) is to establish Theorem 2.5, which states that for any fixed permutation $\pi$ and any fixed assignment $\sigma$ the triple $(\boldsymbol{\Phi}, \pi, \sigma)$ is $\left(\delta_{t}, t\right)$-balanced with probability very close to 1 . The basic symmetry properties of the random formula $\Phi$ allow us to assume without loss of generality that $\pi=\mathrm{id}$ is the identity and that $\sigma=\mathbf{1}$ is the all-true assignment. More precisely, we observe the following:

FACT 3.1. Fix any permutation $\pi$ of $[n]$ and any assignment $\sigma \in\{ \pm 1\}^{V}$. Then, for any $0 \leq t \leq \hat{t}$ we have

$$
\mathrm{P}\left[(\boldsymbol{\Phi}, \pi, \sigma) \text { is }\left(\delta_{t}, t\right) \text {-balanced }\right]=\mathrm{P}\left[(\boldsymbol{\Phi}, \mathrm{id}, \mathbf{1}) \text { is }\left(\delta_{t}, t\right) \text {-balanced }\right] .
$$

Proof. For a $k$-CNF $\Phi$ let $\Phi^{\pi, \sigma}$ be the formula obtained by replacing

—each occurrence of the literal $x_{i}$ in $\Phi$ by $x_{\pi(i)}$ if $\sigma\left(x_{\pi(i)}\right)=1$, and by $\neg x_{\pi(i)}$ if $\sigma\left(x_{\pi(i)}\right)=-1$, and

- each occurrence of the literal $\neg x_{i}$ in $\Phi$ by $\neg x_{\pi(i)}$ if $\sigma\left(x_{\pi(i)}\right)=1$, and by $x_{\pi(i)}$ if $\sigma\left(x_{\pi(i)}\right)=-1$.

Then $(\Phi$, id, $\mathbf{1})$ is $\left(\delta_{t}, t\right)$-balanced if and only if $\left(\Phi^{\pi, \sigma}, \pi, \sigma\right)$ is. Furthermore, the map $\Phi \mapsto \Phi^{\pi, \sigma}$ is a bijection. Consequently, for the uniformly random formula $\Phi$, the resulting formula $\boldsymbol{\Phi}^{\pi, \sigma}$ is uniformly random as well, for any $\pi, \sigma$.

Thus, we assume from now on that $\pi=$ id and $\sigma=\mathbf{1}$. Then, the decimated formula $\boldsymbol{\Phi}_{t, \pi, \sigma}$ is simply obtained from $\boldsymbol{\Phi}$ by substituting the value "true" for $x_{1}, \ldots, x_{t}$ and simplifying. To unclutter the notation, we are going to denote $\boldsymbol{\Phi}_{t, \pi, \sigma}$ by $\boldsymbol{\Phi}^{t}$ from now on. Let $G$ be the factor graph of $\Phi^{t}$.

Our task is to study the BP operator defined in Equations (2) and (3) on $\boldsymbol{\Phi}^{t}$. That is, starting from the initial set of messages $\mu_{x \rightarrow a}^{[0]}( \pm 1)=\frac{1}{2}$, for all $x \in V_{t}, a \in N(x)$, we define inductively for $\ell \geq 0$

$$
\mu_{a \rightarrow x}^{[\ell]}(\zeta)=\left\{\begin{array}{cl}
1 & \text { if } \zeta=\operatorname{sign}(x, a), \\
1-\prod_{y \in N(a) \backslash\{x\}} \mu_{y \rightarrow a}^{[\ell]}(-\operatorname{sign}(y, a)) & \text { if } \zeta=-\operatorname{sign}(x, a)
\end{array}\right.
$$


and

$$
\mu_{x \rightarrow a}^{[\ell+1]}(\zeta)=\operatorname{BP}\left(\mu^{[\ell]}\right)=\frac{\prod_{b \in N(x) \backslash\{a\}} \mu_{b \rightarrow x}^{[\ell]}(\zeta)}{\prod_{b \in N(x) \backslash\{a\}} \mu_{b \rightarrow x}^{[\ell]}(-1)+\prod_{b \in N(x) \backslash\{a\}} \mu_{b \rightarrow x}^{[\ell]}(1)},
$$

unless the denominator equals zero, in which case $\mu_{x \rightarrow a}^{[\ell+1]}(\zeta)=\frac{1}{2}$.

3.1.1. A Nonrigorous Sketch of a Rigorous Analysis. Before launching into the details of the (long and technical) proof, we are going to give a brief sketch based on heuristic considerations. The aim of this is to develop some intuition. Roughly speaking, Theorem 2.5 asserts that with probability very close to 1 , most of the messages $\mu_{x \rightarrow a}^{[\ell]}( \pm 1)$ are close to $1 / 2$. Hence, letting

$$
\Delta_{x \rightarrow a}^{[\ell]}=\mu_{x \rightarrow a}^{[\ell]}(1)-\frac{1}{2}
$$

we aim to show that $\left|\Delta_{x \rightarrow a}^{[\ell]}\right|$ is small for most $x, a$. The proof of this is by induction on $\ell$. That is, given the $\Delta_{x \rightarrow a}^{[\ell]}$, we need to prove that the biases $\Delta_{x \rightarrow a}^{[\ell+1]}$ do not "blow up." More precisely, let us denote by

$$
\theta=1-t / n
$$

the fraction of unassigned variables. Then, our induction hypothesis is that for all but $\delta_{t} \theta n$ variables we have

$$
\max _{a \in N(a)}\left|\Delta_{x \rightarrow a}^{[\ell]}\right| \leq \delta_{t}=\exp (-c \theta k),
$$

and the goal is to show that the same holds true for $\ell+1$. To establish this, we need to investigate one iteration of the update rules from Equations (27) and (28).

Rewriting Equation (27) in terms of the biases $\Delta_{y \rightarrow a}^{[\ell]}$, we obtain

$$
\begin{aligned}
\mu_{a \rightarrow x}^{[\ell]}(-\operatorname{sign}(x, a)) & =1-\prod_{y \in N(a) \backslash\{x\}} \frac{1}{2}-\operatorname{sign}(y, a) \Delta_{y \rightarrow a}^{[\ell]} \\
& =1-2^{1-|N(a)|} \prod_{y \in N(a) \backslash\{x\}} 1-2 \operatorname{sign}(y, a) \Delta_{y \rightarrow a}^{[\ell]} .
\end{aligned}
$$

How many factors do we expect the product in Equation (30) to have? In the undecimated formula $\boldsymbol{\Phi}$, each clause has length $k$. But in $\boldsymbol{\Phi}^{t}$, only a $\theta$ fraction of variables remain unassigned. Hence, the average length of a clause of $\Phi^{t}$ should be $\theta k$. If indeed $|N(a)| \leq 10 \theta k$, say, and if $\left|\Delta_{y \rightarrow a}^{[\ell]}\right| \leq \delta_{t}=\exp (-c \theta k)$ for all $y \in N(a) \backslash\{x\}$, then we can approximate Equation (30) by

$$
\begin{aligned}
\mu_{a \rightarrow x}^{[\ell]}(-\operatorname{sign}(x, a)) & =1-2^{1-|N(a)|} \prod_{y \in N(a) \backslash\{x\}} 1-2 \operatorname{sign}(y, a) \Delta_{y \rightarrow a}^{[\ell]} \\
& \approx 1-2^{1-|N(a)|} \exp \left(\begin{array}{c}
-2 \sum \operatorname{sign}(y, a) \Delta_{y \rightarrow a}^{[\ell]} \\
y \in N(a) \backslash\{x\}
\end{array}\right) \\
& \approx 1-2^{1-|N(a)|}\left(\begin{array}{c}
1-2 \sum_{y \in N(a) \backslash\{x\}} \operatorname{sign}(y, a) \Delta_{y \rightarrow a}^{[\ell]} \\
\text { (l) }
\end{array}\right) .
\end{aligned}
$$

Assume, furthermore, that $a$ is "not too short"-say, $|N(a)| \geq 0.1 \theta k$. Then, $2^{1-|N(a)|} \leq$ $2^{1-0.1 \theta k}$ is small and thus the expression in Equation (31) is close to 1 . Hence, we can 
approximate it by

$$
\mu_{a \rightarrow x}^{[\ell]}(-\operatorname{sign}(x, a)) \approx \exp \left[-2^{1-|N(a)|}\left(1-2 \sum_{y \in N(a) \backslash\{x\}} \operatorname{sign}(y, a) \Delta_{y \rightarrow a}^{[\ell]}\right)\right] .
$$

To proceed, we are going to plug Equation (32) into (28) to estimate $\Delta_{x \rightarrow a}^{[\ell+1]}$. While it is easy enough to multiply the exponentials from Equation (32) together to approximate the numerator of Equation (28), the denominator seems a bit unwieldy. To sidestep this issue, we simply estimate the ratio $\mu_{x \rightarrow a}^{[\ell+1]}(1) / \mu_{x \rightarrow a}^{[\ell+1]}(-1)$ (assuming that $\mu_{x \rightarrow a}^{[\ell+1]}(-1)>0$ ). The denominator cancels. Since $\mu_{x \rightarrow a}^{[\ell+1]}(1)+\mu_{x \rightarrow a}^{[\ell+1]}(-1)=1$ by construction, we see that

$$
\frac{\mu_{x \rightarrow a}^{[\ell+1]}(1)}{\mu_{x \rightarrow a}^{[\ell+1]}(-1)}=\frac{1+2 \Delta_{x \rightarrow a}^{[\ell+1]}}{1-2 \Delta_{x \rightarrow a}^{[\ell+1]}} .
$$

Hence, to show that $\Delta_{x \rightarrow a}^{[\ell+1]}$ is close to zero, it suffices to prove that $\mu_{x \rightarrow a}^{[\ell+1]}(1) / \mu_{x \rightarrow a}^{[\ell+1]}(-1)$ is close to 1 . To this end, we invoke Equation (32), obtaining

$$
\begin{aligned}
\frac{\mu_{x \rightarrow a}^{[\ell+1]}(1)}{\mu_{x \rightarrow a}^{[\ell+1]}(-1)} & =\prod_{b \in N(x) \backslash\{a\}} \frac{\mu_{b \rightarrow x}^{[\ell]}(1)}{\mu_{b \rightarrow x}^{[\ell]}(-1)} \\
& \approx \exp \left[\sum_{b \in N(x) \backslash\{a\}} 2^{1-|N(b)|}\left(\operatorname{sign}(x, b)-2 \sum \operatorname{sign}(x, b) \operatorname{sign}(y, b) \Delta_{y \rightarrow b}^{[\ell]}\right)\right] .
\end{aligned}
$$

Thus, we need to show that for all but $\delta \theta n$ variables $x$ the exponent is close to zero.

To deal with the $\sum_{b \in N(x) \backslash\{a\}} 2^{1-|N(b)|} \operatorname{sign}(x, b)$ bit, we need to estimate in how many clauses of a given length $x$ is likely to appear. Letting

$$
\rho=k r / 2^{k},
$$

we find that the expected number of clauses of length $j$ where $x \in V_{t}$ appears is asymptotically equal to

$$
\frac{k m}{n}\left(\begin{array}{c}
k-1 \\
j-1
\end{array}\right) \theta^{j-1}\left(\frac{1-\theta}{2}\right)^{k-j}=\rho 2^{j} \cdot \mathrm{P}[\operatorname{Bin}(k-1, \theta)=j-1] \leq \rho 2^{j} .
$$

Indeed, the expected number of clauses of $\Phi$ that $x$ appears in equals $k m / n=k r=2^{k} \rho$. Furthermore, each of these gives rise to a clause of length $j$ in $\Phi^{t}$ if and only if exactly $j-1$ among the other $k-1$ variables in the clause are from $V_{t}$, while the $k-j$ remaining variables are in $V \backslash V_{t}$ and occur with negative signs. (If one of them had a positive sign, the clause would have been satisfied by setting the corresponding variable to true. It would thus not be present in $\boldsymbol{\Phi}^{t}$ anymore.) Since $x$ appears with a random sign in each of these clauses, the sum

$$
\sum_{b \in N(x) \backslash\{a\}:|N(b)|=j} \operatorname{sign}(x, b)
$$

can be viewed as a random walk with an expected length of $\rho 2^{j}$. Thus, we expect an outcome of $O\left(\sqrt{2^{j} \rho}\right)$. In this case, we find that

$$
\sum_{b \in N(x) \backslash\{a\}:|N(b)|=j} 2^{1-|N(b)|} \operatorname{sign}(x, b)=2^{1-j} \cdot O\left(\sqrt{2^{j} \rho}\right)=O\left(\sqrt{\rho} 2^{-j / 2}\right) .
$$

Together with the Chernoff bound, Equation (35) shows that $x$ is unlikely to occur in clauses of lengths less than $0.1 \theta k$ or more than $10 \theta k$. Furthermore, our assumption 
that $\theta k \geq \ln (\rho) / c^{2}$ implies that $\sqrt{\rho} 2^{-j / 2} \leq \exp (-0.01 \theta k)$ for all $j \geq 0.1 \theta k$. Hence, we expect that for all but, say, $\delta_{t} \theta n / 2$ variables $x \in V_{t}$

$$
\max _{a \in N(x)}\left|\sum_{b \in N(x) \backslash\{a\}} 2^{1-|N(b)|} \operatorname{sign}(x, b)\right| \leq O(\theta k \exp (-0.01 \theta k)) \leq \delta_{t} / 4 .
$$

The second contribution

$$
\sum_{b \in N(x) \backslash\{a\}} \sum_{y \in N(b) \backslash\{x\}} 2^{2-|N(b)|} \operatorname{sign}(x, b) \operatorname{sign}(y, b) \Delta_{y \rightarrow b}^{[\ell]}
$$

is a linear function of the bias vector $\Delta^{[\ell]}$ from the previous round. Indeed, this operator can be represented by a matrix

$$
\begin{aligned}
& \Lambda^{*}=\left(\Lambda_{x \rightarrow a, y \rightarrow b}^{*}\right)_{x \rightarrow a, y \rightarrow b} \quad \text { with entries } \\
& \Lambda_{x \rightarrow a, y \rightarrow b}^{*}=\left\{\begin{array}{cl}
2^{2-|N(b)|} \operatorname{sign}(x, b) \operatorname{sign}(y, b) & \text { if } a \neq b, x \neq y, \\
0 & \text { otherwise. }
\end{array}\right.
\end{aligned}
$$

with $x \rightarrow a, y \rightarrow b$ ranging over all edges of the factor graph of $\boldsymbol{\Phi}^{t}$.

Since $\Lambda^{*}$ is based on $\boldsymbol{\Phi}^{t}$, it is a random matrix. One could therefore try to use standard arguments to bound it in some norm (say, $\left\|\Lambda^{*}\right\|_{\square}$ ). The problem with this approach is that $\Lambda^{*}$ is very high-dimensional: It operates on a space whose dimension is equal to the number of edges of the factor graph. In effect, standard random matrix arguments do not apply.

To resolve this problem, consider a "projection" of $\Lambda^{*}$ onto a space of dimension merely $\left|V_{t}\right|=\theta n$, namely

$$
\Lambda: \mathbf{R}^{V_{t}} \rightarrow \mathbf{R}^{V_{t}}, \quad \Gamma=\left(\Gamma_{y}\right)_{y \in V_{t}} \mapsto\left\{\sum_{b \in N(x)} \sum_{y \in N(b) \backslash\{x\}} 2^{2-|N(b)|} \operatorname{sign}(x, b) \operatorname{sign}(y, b) \Gamma_{y}\right\}_{x \in V_{t}}
$$

One can think of $\Lambda$ as a signed and weighted adjacency matrix of $\boldsymbol{\Phi}^{t}$. Standard arguments easily show that $\|\Lambda\|_{\square} \leq \delta_{t}^{4} \theta n$ is small with a very high probability. In effect, we expect that for all but, say, $\delta_{t} \theta n / 2$ variables $x \in V_{t}$, we have

$$
\max _{a \in N(x)}\left|\sum_{b \in N(x) \backslash\{a\}} \sum_{y \in N(b) \backslash\{x\}} 2^{2-|N(b)|} \operatorname{sign}(x, b) \operatorname{sign}(y, b) \Delta_{y \rightarrow b}^{[\ell]}\right| \leq \delta_{t} / 4 .
$$

Combining Equations (36) and (37), we thus expect that for all but $\delta_{t} \theta n$ variables $x$ the expression in Equation (33) is sufficiently close to 1 to conclude that $\max _{a \in N(x)}\left|\Delta_{x \rightarrow a}^{[\ell+1]}\right| \leq$ $\delta_{t}$, thereby completing the induction.

3.1.2. Rigorizing the Sketch. While the preceding outlines a strategy for tracing the BP operator, we clearly glossed over numerous issues. The remainder of this article is devoted to rectifying them. To provide a bit of orientation, we briefly highlight the most important items and indicate how they are going to be fixed.

The first issue is that Theorem 2.5 claims a rather strong bound on the probability that $\boldsymbol{\Phi}^{t}$ is $\left(\delta_{t}, t\right)$-balanced. To obtain this bound, we are going to proceed in two steps: In Section 3.2, we will exhibit a small number quasi-random properties and show that these hold in $\boldsymbol{\Phi}^{t}$ with the required probability. Then, in Section 3.3, we are going to show deterministically that any formula that has these properties is $\left(\delta_{t}, t\right)$-balanced.

A second major issue is the $\approx$ signs in the preceding discussion. Their use depended on the assumption that $\left|\Delta_{x \rightarrow a}^{[\ell]}\right| \leq \delta_{t}$ for all $x \in V_{t}, a \in N(x)$. However, this assumption 
is not going to be valid for any $\ell \geq 1$. Indeed, for some $x, \max _{a \in N(x)}\left|\Delta_{x \rightarrow a}^{[\ell]}\right|$ is going to be close or even equal to 1/2: Think of a variable that appears in a clause of length one (a "unit clause"), or of a variable of a very high degree that appears only positively (a "pure literal"). Hence, we will need to cope with a small but non-empty set $T[\ell]$ of "exceptional" variables $x$ with $\max _{a \in N(x)}\left|\Delta_{x \rightarrow a}^{[\ell]}\right|>\delta_{t}$.

To study the impact of the exceptional set, we decompose Equation (30) as

$$
\begin{aligned}
\mu_{a \rightarrow x}^{[\ell]}(-\operatorname{sign}(x, a))=1-2^{1-|N(a)|} & {\left[\prod_{y \in N(a) \backslash(T[\ell] \cup\{x\})} 1-2 \operatorname{sign}(y, a) \Delta_{y \rightarrow a}^{[\ell]}\right] } \\
& \cdot\left[\prod_{y \in N(a) \cap T[\ell] \backslash\{x\}} 1-2 \operatorname{sign}(y, a) \Delta_{y \rightarrow a}^{[\ell]}\right] .
\end{aligned}
$$

There are going to be various cases depending on the length of the clause. If, say, $0.1 \theta k \leq|N(a)| \leq 10 \theta k$ and $|N(a) \cap T[\ell] \backslash\{x\}| \leq 1$ (i.e., the second product contains at most one factor), then the preceding heuristic computation essentially goes through. This case is going to be represented by the set $N_{\leq 1}(x, T[\ell])$ below.

More generally, if $0.1 \theta k \leq|N(a)| \leq 10 \theta k$, say, then the product of Equation (38) is quite close to 1 , regardless of $|N(a) \cap T[\ell] \backslash\{x\}|$. Thus, a single "exposed" clause $a$, or even a small number, are not going to affect the ratio in Equation (33) much. To exploit this, we will establish as part of the quasi-randomness property that for any possible set $T[\ell]$ only very few variables $x$ are "heavily exposed," meaning that they appear in many clauses that contain several variables from $T[\ell]$ (cf. Q2 and Q3 later). Furthermore, we will generally show that there are only very few variables that occur in a clause $a$ such that $|N(a)| \notin[0.1 \theta k, 10 \theta k]$ (cf. Q1).

A third issue is the dimension reduction in the linear operator (i.e., that we work with $\Lambda$ instead of $\Lambda^{*}$ ). To vindicate this point, we need to show that for most variables $x$ the bias $\Delta_{x \rightarrow a}^{[\ell]}$ is essentially independent of $a$. Furthermore, we need to modify the operator $\Lambda$ to "cut out" the exceptional set $T[\ell]$ where the BP operator has a highly nonlinear behavior. This is going to be mirrored in condition $\mathbf{Q} 4$ below.

Let us now turn this sketch into an actual proof. In Section 3.2, we introduce the quasi-randomness property and state the deterministic result about BP on quasirandom formulas (Theorem 3.4). Then, from Section 3.3 onward, we prove Theorem 3.4. Finally, in Section 4, we establish that the quasi-randomness property holds on $\Phi^{t}$ with the required probability.

\subsection{The Quasirandomness Property}

In this section, we exhibit a few simple quasi-randomness properties that $\boldsymbol{\Phi}^{t}$ is very likely to possess. From Section 3.3 onward, we will show that these properties suffice to trace the $\mathrm{BP}$ operator.

To state the quasi-randomness properties, fix a $k$-CNF $\Phi$. Let $\Phi^{t}=\Phi_{t, \text { id, } 1}$ denote the CNF obtained from $\Phi$ by substituting "true" for $x_{1}, \ldots, x_{t}$ and simplifying $(1 \leq t \leq n)$. Let $V_{t}=\left\{x_{t+1}, \ldots, x_{n}\right\}$ be the set of variables of $\Phi^{t}$. As before, we will denote the factor graph of $\Phi^{t}$ by $G=G\left(\Phi^{t}\right)$ and the neighborhood of a vertex $v$ by $N(v)$. We continue to let $\theta$ and $\rho$ be defined as in Equations (29) and (34) and we set $\delta=\delta_{t}$.

For a variable $x \in V_{t}$ and a set $T \subset V_{t}$ let

$$
N_{\leq 1}(x, T)=\{b \in N(x):|N(b) \cap T \backslash\{x\}| \leq 1 \wedge 0.1 \theta k \leq|N(b)| \leq 10 \theta k\} .
$$

Thus, $N_{\leq 1}(x, T)$ is the set of all clauses that contain $x$ (which may or may not be in $T$ ) and at most one other variable from $T$. In addition, there is a condition on the length 
Q0. $\Phi$ is tame.

Q1. No more than $10^{-5} \delta \theta n$ variables occur in clauses of length less than $\theta k / 10$ or greater than $10 \theta k$ in $\Phi^{t}$. Moreover, there are at most $10^{-4} \delta \theta n$ variables $x \in V_{t}$ such that

$$
(\theta k)^{3} \delta \cdot \sum_{b \in N(x)} 2^{-|N(b)|}>1 .
$$

Q2. If $T \subset V_{t}$ has size $|T| \leq \delta \theta n$, then there are no more than $10^{-4} \delta \theta n$ variables $x$ such that either

$$
\begin{aligned}
& \sum_{b \in N_{1}(x, T)} 2^{-|N(b)|}>\rho(\theta k)^{5} \delta, \text { or } \sum_{b \in N_{>1}(x, T)} 2^{|N(b) \cap T \backslash\{x\}|-|N(b)|}>\frac{\delta}{\theta k} \text {, or } \\
& \left|\sum_{b \in N_{\leq 1}(x, T)} \frac{\operatorname{sign}(x, b)}{2^{|N(b)|}}\right|>\frac{\delta}{1000} .
\end{aligned}
$$

Q3. For any $0.01 \leq z \leq 1$ and any set $T \subset V_{t}$ of size $|T| \leq 100 \delta \theta n$ we have

$$
\sum_{b:|N(b) \cap T| \geq z|N(b)|}|N(b)| \leq \frac{1.01}{z}|T|+10^{-4} \delta \theta n .
$$

Q4. For any set $T \subset V_{t}$ of size $|T| \leq 10 \delta \theta n$ the linear operator $\Lambda_{T}: \mathbf{R}^{V_{t}} \rightarrow \mathbf{R}^{V_{t}}$,

$$
\Gamma=\left(\Gamma_{y}\right)_{y \in V_{t}} \mapsto\left\{\sum_{b \in N_{\leq 1}(x, T)} \sum_{y \in N(b) \backslash\{x\}} 2^{-|N(b)|} \operatorname{sign}(x, b) \operatorname{sign}(y, b) \Gamma_{y}\right\}_{x \in V_{t}}
$$

has norm $\left\|\Lambda_{T}\right\|_{\square} \leq \delta^{4} \theta n$.

Fig. 2. The conditions for Definition 3.2.

$|N(b)|$ of the clause $b$ in the decimated formula $\Phi_{t}$. Recall from Section 3.1 that having assigned the first $t$ variables, we should expect the average clause length to be $\theta k$.

With $c>0$ as in (14) we let

$$
k_{1}=\sqrt{c} \theta k .
$$

Moreover, for a variable $x \in V_{t}$ and a set $T \subset V_{t}$ let

$$
\begin{aligned}
N_{1}(x, T) & =\left\{b \in N(x):|N(b) \backslash T| \geq k_{1},|N(b) \cap T \backslash\{x\}|=1\right\}, \\
N_{>1}(x, T) & =\left\{b \in N(x):|N(b) \backslash T| \geq k_{1},|N(b) \cap T \backslash\{x\}|>1\right\} .
\end{aligned}
$$

Definition 3.2. Let $\delta>0$. We say that $\Phi$ is $(\delta, t)$-quasi-random if Q0-Q4 in Figure 2 are satisfied.

Condition Q0 simply bounds the number of redundant clauses and the number of variables of very high degree; it is well-known to hold for random $k$-CNFs with high probability. Apart from a bound on the number of very short/very long clauses, Q1 provides a bound on the weight of clauses in which variables $x \in V_{t}$ typically occur, where the weight of a clause $b$ is $2^{-|N(b)|}$. Moreover, Q2 provides that there is no small set $T$ for which the total weight of the clauses touching that set is very big. In addition, Q2 (essentially) requires that, for most variables, $x$ the weights of the clauses where $x$ occurs positively/negatively should approximately cancel. Furthermore, Q3 provides a bound on the lengths of clauses that contain many variables from a small set $T$. 
Finally, the most important condition is $\mathbf{Q 4}$, providing a bound on the cut norm of a signed, weighted matrix representation of $\Phi^{t}$.

Proposition 3.3. There exists a constant $\rho_{0}>0$ such that for any $k, r$ satisfying $\rho_{0} \cdot 2^{k} / k \leq r \leq 2^{k} \ln 2$ there is $0<\xi=\xi(k, r) \leq 1 / k$ so that for $n$ large and $\delta_{t}$, $\hat{t}$ as in Equation (14) for any $1 \leq t \leq \hat{t}$ we have

$$
\mathrm{P}\left[\boldsymbol{\Phi} \text { is }\left(\delta_{t}, t\right) \text {-quasirandom } \mid \boldsymbol{\Phi} \text { is tame }\right] \geq 1-\exp \left[-10\left(\xi n+\Delta_{t}\right)\right] .
$$

The proof of Proposition 3.3 is a necessary evil: It is long, complicated, and based on standard arguments. We defer it to Section 4. Together with the following theorem, which we will establish in Section 3.3, Proposition 3.3 yields Theorem 2.5.

THEOREM 3.4. There is $\rho_{0}>0$ such that for any $k, r$ satisfying $\rho_{0} \cdot 2^{k} / k \leq r \leq 2^{k} \ln 2$ and $n$ sufficiently large the following is true. Let $\Phi$ be a $k$-CNF with $n$ variables and $m$ clauses that is $\left(\delta_{t}, t\right)$-quasi-random for some $1 \leq t \leq \hat{t}$. Then $(\Phi, \mathrm{id}, \mathbf{1})$ is $\left(\delta_{t}, t\right)$-balanced.

The rest of this section deals with the proof of Theorem 3.4.

For the rest of Section 3, we keep the notation from Section 3.2 and the assumptions of Theorem 3.4. To unclutter the notation, we continue to let $\delta=\delta_{t}$.

\subsection{Belief Propagation on Quasi-Random Formulas: Proof of Theorem 3.4}

Implementing the strategy outlined in Section 3.1, we trace the BP operator when iterated from the initial point

$$
\mu_{x \rightarrow a}^{[0]}(-1)=\mu_{x \rightarrow a}^{[0]}(1)=\frac{1}{2} \quad \text { for all } x \in V_{t}, a \in N(x) .
$$

Let $\mu^{[\ell]}=\mathrm{BP}^{\ell}(\mu[0]) \in M(\Phi)$ be the result of the first $\ell$ iterations of BP. Let

$$
\Delta_{x \rightarrow a}^{[\ell]}=\mu_{x \rightarrow a}^{[\ell]}(1)-\frac{1}{2}
$$

We say that $x \in V_{t}$ is $\ell$-biased if

$$
\max _{a \in N(x)}\left|\Delta_{x \rightarrow a}^{[\ell]}\right| \geq 0.1 \delta
$$

Clearly, no variable is 0 -biased. Let $B[\ell]$ be the set of all $\ell$-biased variables. To prove Theorem 3.4, the core task will be to bound $|B[\ell]|$.

To this end, we are going to construct a sequence of sets $T[\ell]$ whose sizes are easier to estimate and that will turn out to be supersets of the $B[\ell]$. Actually, we will construct sets of variables $T_{1}[\ell], T_{2}[\ell]$ and sets of clauses $T_{3}[\ell]$ inductively and let $T[\ell]=T_{1}[\ell] \cup$ $T_{2}[\ell] \cup N\left(T_{3}[\ell]\right)$.

For $\ell=0$, we let $T_{1}[0]=T_{3}[0]=\emptyset$. Moreover, let $T_{2}[0]$ be the set of all variables $x$ such that there is a clause $b \in N(x)$ that is either redundant, or $|N(b)|<0.1 \theta k$, or $|N(b)|>10 \theta k$, or that satisfies $\delta(\theta k)^{3} \sum_{b \in N(x)} 2^{-|N(b)|}>1$.

To define $T[\ell+1]$ inductively for $\ell \geq 0$, we need a bit of notation: For $x \in V$ and $a \in N(x)$ we let

$$
N_{\leq 1}^{[\ell+1]}(x \rightarrow a)=\left\{b \in N_{\leq 1}(x, T[\ell]) \backslash\{a\}: \mu_{b \rightarrow x}^{[\ell]}(-1)>0\right\} .
$$

Furthermore, set

$$
P_{\leq 1}^{[\ell+1]}(x \rightarrow a)=\prod_{b \in N_{\leq 1}^{[\ell+1]}(x \rightarrow a)} \frac{\mu_{b \rightarrow x}^{[\ell]}(1)}{\mu_{b \rightarrow x}^{[\ell]}(-1)},
$$


In addition, let

$$
\begin{aligned}
& N_{>1}^{[\ell+1]}(x \rightarrow a)=\left\{b \in N(x) \backslash\left(\{a\} \cup N_{\leq 1}(x, T[\ell])\right): \mu_{b \rightarrow x}^{[\ell]}(-1)>0\right\}, \\
& P_{>1}^{[\ell+1]}(x \rightarrow a)=\prod_{b \in N_{>1}^{[\ell+1]}(x \rightarrow a)} \frac{\mu_{b \rightarrow x}^{[\ell]}(1)}{\mu_{b \rightarrow x}^{[\ell]}(-1)} .
\end{aligned}
$$

The motivation behind these definitions is the following. Assume for a moment that $\mu_{b \rightarrow x}^{[\ell]}(-1) \neq 0$ for all $b \in N(x)$. As we saw in Section 3.1, to show that $\Delta_{x \rightarrow a}^{[\ell+1]}=\mu_{x \rightarrow a}^{[\ell+1]}(1)-\frac{1}{2}$ is close to zero it suffices to verify that the ratio

$$
\frac{\mu_{x \rightarrow a}^{[\ell+1]}(1)}{\mu_{x \rightarrow a}^{[\ell+1]}(-1)}=\prod_{b \in N(x) \backslash\{a\}} \frac{\mu_{b \rightarrow x}^{[\ell]}(1)}{\mu_{b \rightarrow x}^{[\ell]}(-1)}=P_{\leq 1}^{[\ell+1]}(x \rightarrow a) \cdot P_{>1}^{[\ell+1]}(x \rightarrow a)
$$

is close to 1 , because $\mu_{x \rightarrow a}^{[\ell+1]}(-1)+\mu_{x \rightarrow a}^{[\ell+1]}(1)=1$ by construction. Moreover, Equation (41) is close to 1 if both factors on the right-hand side are.

Now, we let $T_{1}[\ell+1]$ contain all variables for which $P_{\leq 1}^{[\ell+1]}(x \rightarrow a)$ fails to be close enough to one:

$$
T_{1}[\ell+1]=\left\{x \in V: \max _{a \in N(x)}\left|P_{\leq 1}^{[\ell+1]}(x \rightarrow a)-1\right|>0.01 \delta\right\} .
$$

To also deal with the second product $P_{>1}^{[\ell+1]}(x \rightarrow a)$, we define additional sets $T_{2}[\ell+1]$, $T_{3}[\ell+1]$. To define $T_{2}[\ell+1]$, let us say that a variable $x$ is $(\ell+1)$-harmless if it enjoys the following four properties:

H1. We have $\delta(\theta k)^{3} \sum_{b \in N(x)} 2^{-|N(b)|} \leq 1$, and $0.1 \theta k \leq|N(b)| \leq 10 \theta k$ for all $b \in N(x)$.

H2. $\sum_{b \in N_{1}(x, T[\ell])} 2^{-|N(b)|} \leq \rho(\theta k)^{5} \delta$ and

$$
\sum_{b \in N_{>1}(x, T[\ell])} 2^{|N(b) \cap T[\ell] \backslash\{x\}|-|N(b)|} \leq \delta /(\theta k) .
$$

H3. There is at most one clause $b \in N(x)$ such that $|N(b) \backslash T[\ell]| \leq k_{1}$.

H4. $\left|\sum_{b \in N_{\leq 1}(x)} \operatorname{sign}(x, b) \cdot 2^{-|N(b)|}\right| \leq 0.01 \delta$.

Let $H[0]=V_{t}$ and let $H[\ell+1]$ signify the set of all $(\ell+1)$-harmless variables for $\ell \geq 0$. Furthermore, let $T_{2}[\ell+1]$ be the set of all variables $x$ that have at least one of the following properties:

T2a.There is a clause $b \in N(x)$ that is either redundant, or

$$
|N(b)|<0.1 \theta k, \text { or }|N(b)|>10 \theta k \text {. }
$$

T2b. $\delta(\theta k)^{3} \sum_{b \in N(x)} 2^{-|N(b)|}>1$.

T2c. Either

$$
\begin{aligned}
\sum_{b \in N_{1}(x, T[\ell])} 2^{-|N(b)|} & >\rho(\theta k)^{5} \delta, \quad \text { or } \\
\sum_{b \in N_{>1}(x, T[\ell])} 2^{|N(b) \cap T[\ell] \backslash\{x\}|-|N(b)|} & >\delta /(\theta k) .
\end{aligned}
$$

T2d. $x$ occurs in more than 100 clauses from $T_{3}[\ell]$.

T2e. $x$ occurs in a clause $b$ that contains fewer than $3|N(b)| / 4$ variables from $H[\ell]$. 
Items $\mathbf{Q 0}$ and $\mathbf{Q 1}$ from Definition 3.2 ensure that there are only a very few variables that violate $\mathbf{H 1}$ or satisfy $\mathbf{T} 2 \mathbf{a}$ or $\mathbf{T} \mathbf{2 b}$. We always include these few in the set $T_{2}[\ell+1]$ of "exceptional" variables. Moreover, intuitively, H2 and T2c-T2e capture variables $x$ that are highly exposed to the exceptiona' set $T[\ell]$ from the previous round. Furthermore, we let

$$
T_{3}[\ell+1]=\left\{a \in \Phi^{t}:|N(a)| \geq 100 k_{1} \wedge|N(a) \backslash T[\ell]| \leq k_{1}\right\} \backslash T_{3}[\ell]
$$

contain all clauses that consist almost entirely of exceptional variables from $T[\ell]$ but without including the clauses from the previous set $T_{3}[\ell]$. Finally,

$$
T[\ell+1]=T_{1}[\ell+1] \cup T_{2}[\ell+1] \cup N\left(T_{3}[\ell+1]\right) .
$$

In Section 3.4, we will verify that $T[\ell]$ does indeed contain the set $B[\ell]$ of biased variables.

Proposition 3.5. We have $B[\ell] \subset T[\ell]$ for all $\ell \geq 0$.

Furthermore, in Section 3.5, we will establish the following bound on the size of $T[\ell]$ :

Proposition 3.6. We have $|T[\ell]|<\delta \theta n$ for all $\ell \geq 0$.

Finally, in Section 3.8, we will derive Theorem 3.4 from Propositions 3.5 and 3.6.

\subsection{Proof of Proposition 3.5}

The proof will be by induction on $\ell$. We begin with an elementary estimate of the messages $\mu_{b \rightarrow x}$ from clauses to variables:

LemMa 3.7. Let $x$ be a variable and let $b \in N(x)$ be a clause. Let

$$
t_{b}=|N(b) \cap B[\ell] \backslash\{x\}|
$$

Then

$$
0 \leq 1-\mu_{b \rightarrow x}^{[\ell]}(\zeta) \leq 2^{2-|N(b)|+t_{b}} \exp (\delta|N(b)|) \quad \text { for } \zeta= \pm 1 .
$$

Furthermore, if $2^{2-|N(b)|+t_{b}} \exp (\delta|N(b)|) \mid \leq 1 / 2$, then

$$
\exp \left[-2^{3-|N(b)|+t_{b}} \exp (\delta|N(b)|)\right] \leq \mu_{b \rightarrow x}^{[\ell]}(\zeta) \leq 1 \quad \text { for } \zeta= \pm 1
$$

Proof. Since for any $y \in N(b) \backslash\{x\}$ we have $\mu_{y \rightarrow b}^{[\ell]}(1)=\frac{1}{2}+\Delta_{y \rightarrow b}^{[\ell]}$ and $\mu_{y \rightarrow b}^{[\ell]}(-1)+$ $\mu_{y \rightarrow b}^{[\ell]}(1)=1$, we see that

$$
\mu_{y \rightarrow b}^{[\ell]}(-\operatorname{sign}(y, b))=\frac{1}{2}-\operatorname{sign}(y, b) \Delta_{y \rightarrow b}^{[\ell]} .
$$


Therefore, by the definition (27) of $\mu_{b \rightarrow x}^{[\ell]}( \pm 1)$, we have

$$
\begin{aligned}
0 & \leq 1-\mu_{b \rightarrow x}^{[\ell]}(-\operatorname{sign}(x, b))=\prod_{y \in N(b) \backslash\{x\}} \frac{1}{2}-\operatorname{sign}(y, b) \Delta_{y \rightarrow b}^{[\ell]} \\
& =2^{1-|N(b)|} \prod_{y \in N(b) \backslash\{x\}} 1-2 \operatorname{sign}(y, b) \Delta_{y \rightarrow b}^{[\ell]} \\
& \leq 2^{1-|N(b)|} \cdot 2^{t_{b}} \cdot \prod_{y \in N(b) \backslash(\{x\} \cup B[\ell])} 1+2\left|\Delta_{y \rightarrow b}^{[\ell]}\right| \quad \quad\left[\text { as } \Delta_{y \rightarrow b}^{[\ell]} \in[-1 / 2,1 / 2] \text { for all } y\right] \\
& \left.\leq 2^{1-|N(b)|} \cdot 2^{t_{b}} \cdot \exp \left[2 \sum_{y \in N(b) \backslash(\{x\} \cup B[\ell])} \mid \Delta_{y \rightarrow b}^{[\ell]}\right]\right] \quad\left[\text { as }\left|\Delta_{y \rightarrow b}^{[\ell]}\right| \leq 0.1 \delta \text { for all } y \notin B[\ell]\right] .
\end{aligned}
$$

The second assertion follows from the elementary inequality $1-z \geq \exp (-2 z)$ for $0 \leq z \leq 1 / 2$.

Corollary 3.8. Let $x$ be a variable and let $\mathcal{T} \subset N(x)$ be a set of clauses. For each $b \in \mathcal{T}$, let $t_{b}=|N(b) \cap B[\ell] \backslash\{x\}|$. Assume that $t_{b}<|N(b)|-2$ and $|N(b)| \leq 10 \theta k$ for all $b \in \mathcal{T}$. Then, $\mu_{b \rightarrow x}^{[\ell]}( \pm 1)>0$ for all $b \in \mathcal{T}$ and

$$
\left|\ln \prod_{b \in \mathcal{T}} \frac{\mu_{b \rightarrow x}^{[\ell]}(1)}{\mu_{b \rightarrow x}^{[\ell]}(-1)}\right| \leq \sum_{b \in \mathcal{T}} 2^{4-|N(b)|+t_{b}} .
$$

Proof. For each $b \in \mathcal{T}$ there is $y \in N(b) \backslash\{x\}$ such that $y \notin B[\ell]$ because $t_{b}<|N(b)|-2$. Therefore, Equation (27) shows that $\mu_{b \rightarrow x}^{[\ell]}( \pm 1)>0$. Since by definition $\mu_{b \rightarrow x}^{[\ell]}(\zeta) \leq 1$ for $\zeta= \pm 1$, Lemma 3.7 implies that for any $b \in \mathcal{T}$, and we have

$$
\frac{\mu_{b \rightarrow x}^{[\ell]}(\zeta)}{\mu_{b \rightarrow x}^{[\ell]}(-\zeta)} \geq 1-2^{2-|N(b)|+t_{b}} \exp (\delta|N(b)|) .
$$

Our assumptions $t_{b}<|N(b)|-2$ and $|N(b)| \leq 10 \theta k$ ensure that

$$
2^{2-|N(b)|+t_{b}} \leq 1 / 2 \quad \text { and } \quad \exp (\delta|N(b)|) \leq 1.1,
$$

whence $2^{2-|N(b)|+t_{b}} \exp (\delta|N(b)|) \leq 0.6$. Due to the elementary inequality $1-z \geq \exp (-2 z)$ for $z \in[0,0.6]$, Equation (43) thus yields

$$
\frac{\mu_{b \rightarrow x}^{[\ell]}(\zeta)}{\mu_{b \rightarrow i}^{[\ell]}(-\zeta)} \geq \exp \left[-2^{3-|N(b)|+t_{b}} \exp (\delta|N(b)|)\right] \geq \exp \left[-2^{4-|N(b)|+t_{b}}\right]
$$

Multiplying Equation (44) up over $b \in \mathcal{T}$ and taking logarithms yields

$$
\ln \prod_{b \in \mathcal{T}} \frac{\mu_{b \rightarrow x}^{[\ell]}(\zeta)}{\mu_{b \rightarrow x}^{[\ell]}(-\zeta)} \geq-\sum_{b \in \mathcal{T}} 2^{3-|N(b)|+t_{b}} \exp (\delta|N(b)|) .
$$

Since Equation (45) holds for both $\zeta=-1$ and $\zeta=1$, the assertion follows.

Corollary 3.9. Suppose that $x \in H[\ell]$ and that $a \in N(x)$ is a clause such that $|N(a) \backslash T[\ell-1]| \leq k_{1}$. Moreover, assume that $B[\ell-1] \subset T[\ell-1]$. Then $\left|\Delta_{x \rightarrow a}^{[\ell]}\right| \leq 0.01$. 
Proof. For each $b \in N(x) \backslash\{a\}$ let $t_{b}=|N(b) \cap B[\ell-1] \backslash\{x\}|$. Then our assumption that $B[\ell-1] \subset T[\ell-1]$ and condition $\mathbf{H 3}$ ensure that for any $b \in N(x) \backslash\{a\}$,

$$
t_{b} \leq|N(b) \cap T[\ell-1]| \leq|N(b)|-k_{1}<|N(b)|-2 .
$$

Furthermore, by H1, we have $0.1 \theta k \leq|N(b)| \leq 10 k \theta$ for all $b \in N(x) \backslash\{a\}$. Therefore, Corollary 3.8 applies to the set $\mathcal{T}=N_{>1}(x, T[\ell]) \backslash\{a\}$. Since Corollary 3.8 yields $\mu_{b \rightarrow x}^{[\ell-1]}( \pm 1)>0$ for all $b \in \mathcal{T}$, we have $\mathcal{T}=N_{>1}^{[\ell]}(x \rightarrow a)$, and thus

$$
\left|\ln P_{>1}^{[\ell]}(x \rightarrow a)\right|=\left|\ln \prod_{b \in \mathcal{T}} \frac{\mu_{b \rightarrow x}^{[\ell]}(1)}{\mu_{b \rightarrow x}^{[\ell]}(-1)}\right| \leq \sum_{b \in \mathcal{T}} 2^{4-|N(b)|+t_{b}} .
$$

Moreover, H2 ensures that $\sum_{b \in \mathcal{T}} 2^{t_{b}-|N(b)|} \leq \delta$, whence Equation (46) entails

$$
\left|P_{>1}^{[\ell]}(x \rightarrow a)-1\right| \leq 10^{-4} \text {. }
$$

Furthermore, by H1, all clauses $b \in N(x)$ have lengths $0.1 \theta k \leq|N(b)| \leq 10 \theta k$. Moreover, for all $b \in N(x) \backslash\{a\}$ we have $|N(b) \backslash T[\ell-1]| \geq k_{1}$ by H3, and thus $N_{1}(x, T[\ell-1]) \subset N_{\leq 1}(x, T[\ell-1])$. Furthermore, since $|N(a) \cap T[\ell-1]|>1$ by assumption, we have

$$
N_{\leq 1}^{[\ell]}(x \rightarrow a)=N_{\leq 1}(x, T[\ell-1]) .
$$

Hence, letting $\mathcal{N}=N_{\leq 1}(x, T[\ell-1]) \backslash N_{1}(x, T[\ell-1])$, we have

$$
P_{\leq 1}^{[\ell]}(x \rightarrow a)=\prod_{b \in \mathcal{N}} \frac{\mu_{b \rightarrow x}^{[\ell-1]}(1)}{\mu_{b \rightarrow x}^{[\ell-1]}(-1)} \cdot \prod_{b \in N_{1}(x, T[\ell-1])} \frac{\mu_{b \rightarrow x}^{[\ell-1]}(1)}{\mu_{b \rightarrow x}^{[\ell-1]}(-1)} .
$$

With respect to the second product, Corollary 3.8 yields

$$
\begin{aligned}
\left|\ln \prod_{b \in N_{1}(x, T[\ell-1])} \frac{\mu_{b \rightarrow x}^{[\ell-1]}(1)}{\mu_{b \rightarrow x}^{[\ell-1]}(-1)}\right| & \leq \sum_{b \in N_{1}(x, T[\ell-1])} 2^{5-|N(b)|} \\
& \left.\leq 32 \rho(\theta k)^{5} \delta \leq 10^{-6} \text { [as } \delta=\exp (-c \theta k) \text { with } \theta k \geq \ln (\rho) / c^{2}\right] .
\end{aligned}
$$

Furthermore, for any $b \in \mathcal{N}$ we have

$$
\begin{aligned}
\mu_{b \rightarrow x}^{[\ell-1]}(-\operatorname{sign}(x, b)) & =1-\prod_{y \in N(b) \backslash\{x\}} \frac{1}{2}-\operatorname{sign}(y, b) \Delta_{y \rightarrow b}^{[\ell-1]} \\
& =1-2^{1-|N(b)|} \prod_{y \in N(b) \backslash\{x\}} 1-2 \operatorname{sign}(y, b) \Delta_{y \rightarrow b}^{[\ell-1]} .
\end{aligned}
$$

Since $b \in \mathcal{N}$, for all $y \in N(b) \backslash\{x\}$ we have $y \notin B[\ell-1] \subset T[\ell-1]$, and thus $\left|\Delta_{y \rightarrow b}^{[\ell-1]}\right| \leq$ $0.1 \delta$. Moreover, $|N(b)| \leq 10 k \theta$ by $\mathbf{H 1}$. Thus, letting

$$
\alpha_{b}=1-\prod_{y \in N(b) \backslash\{x\}} 1-2 \operatorname{sign}(y, b) \Delta_{y \rightarrow b}^{[\ell-1]},
$$

we find

$$
0 \leq \alpha_{b} \leq 1-(1-0.2 \delta)^{|N(b)|} \leq 8 \delta k \theta .
$$

Since $|N(b)| \geq 0.1 k \theta$ by H1, Equation (50) thus yields

$$
\mu_{b \rightarrow x}^{[\ell-1]}(-\operatorname{sign}(x, b)) \geq 1-2^{1-|N(b)|}(1+\delta k \theta) \geq 0.99 .
$$


Using the elementary inequality $-z-z^{2} \leq \ln (1-z) \leq-z$ for $0 \leq z \leq 0.5$, we obtain from Equations (50), (51), and (52)

$$
\begin{aligned}
\ln \mu_{b \rightarrow x}^{[\ell-1]}(-\operatorname{sign}(x, b)) & \leq-2^{1-|N(b)|}\left(1-\alpha_{b}\right) \leq-2^{1-|N(b)|}(1-8 k \theta \delta), \\
\ln \mu_{b \rightarrow x}^{[\ell-1]}(-\operatorname{sign}(x, b)) & \geq-2^{1-|N(b)|}\left(1-\alpha_{b}\right)-2^{2(1-|N(b)|)}\left(1-\alpha_{b}\right)^{2} \\
& \geq-2^{1-|N(b)|}(1+8 k \theta \delta) \quad[\operatorname{as}|N(b)| \geq 0.1 k \theta \text { by H1] } .
\end{aligned}
$$

Summing these bounds up for $b \in \mathcal{N}$, we obtain

$$
\begin{aligned}
& \left|\ln \prod_{b \in \mathcal{N}} \frac{\mu_{b \rightarrow x}^{[\ell-1]}(1)}{\mu_{b \rightarrow x}^{[\ell-1]}(-1)}\right| \leq\left|\sum_{b \in \mathcal{N}} \operatorname{sign}(x, b) 2^{1-|N(b)|}\right|+8 k \theta \delta \sum_{b \in \mathcal{N}} 2^{1-|N(b)|} \\
& \leq 2\left|\sum_{b \in \mathcal{N}} \operatorname{sign}(x, b) 2^{-|N(b)|}\right|+8(k \theta)^{-2} \quad[\text { by } \mathbf{H 1}] \\
& \leq 2\left|\sum_{b \in N_{\leq 1}(x, T[\ell-1])} \operatorname{sign}(x, b) 2^{-|N(b)|}\right|+8(k \theta)^{-2} \\
& +\sum_{b \in N_{1}(x, T[\ell-1])} 2^{1-|N(b)|} \\
& \leq 0.02 \delta+8(k \theta)^{-2}+\rho(\theta k)^{5} \delta \quad \text { [by H2, H4] } \\
& \leq 10^{-6} \quad \text { [because } \delta=\exp (-c k \theta) \text { and } k \theta \geq \ln (\rho) / c^{2} \text { ]. }
\end{aligned}
$$

Plugging Equations (49) and (53) into (48), we see that $\left|P_{\leq 1}^{[\ell]}(x \rightarrow a)-1\right| \leq 10^{-5}$, while $\left|P_{>1}^{[\ell]}(x \rightarrow a)-1\right| \leq 10^{-4}$ by Equation (47). Therefore, Equation (41) yields

$$
\left|1-\frac{1+2 \Delta_{x \rightarrow a}^{[\ell]}}{1-2 \Delta_{x \rightarrow a}^{[\ell]}}\right|=\left|1-\frac{\mu_{x \rightarrow a}^{[\ell]}(1)}{\mu_{x \rightarrow a}^{[\ell]}(-1)}\right| \leq 3 \cdot 10^{-4},
$$

whence $\left|\Delta_{x \rightarrow a}^{[\ell]}\right| \leq 0.01$, as desired.

Corollary 3.10. Let $b$ be a clause such that $N(b) \not \subset T[\ell]$. Let $x \in N(b)$. Assume that $B[\ell-1] \subset T[\ell-1]$. Then

$$
\mu_{b \rightarrow x}^{[\ell-1]}(-1)>0 \text { and }\left|\frac{\mu_{b \rightarrow x}^{[\ell-1]}(1)}{\mu_{b \rightarrow x}^{[\ell-1]}(-1)}-1\right| \leq \exp \left(-k_{1} / 2\right) \text {. }
$$

Proof. We consider two cases.

Case 1: $|N(b) \backslash T[\ell-1]|>k_{1}$ Since $N(b) \not \subset T[\ell]$, we have $|N(b)| \leq 10 k \theta$ (by T2a). Therefore, Lemma 3.7 yields

$$
\exp \left(-\exp \left(-0.6 k_{1}\right)\right) \leq \exp \left[-2^{3-k_{1}} \exp (\delta|N(b)|)\right] \leq \mu_{b \rightarrow x}^{[\ell-1]}(\zeta) \leq 1 \quad \text { for } \zeta= \pm 1,
$$

whence the assertion follows.

Case 2: $|N(b) \backslash T[\ell-1]| \leq k_{1}$. Since $N(b) \not \subset T[\ell]$, condition T2a ensures that $0.1 \theta k \leq$ $|N(b)| \leq 10 \theta k$. The assumption $N(b) \not \subset T[\ell]$ implies that $b \notin T_{3}[\ell]$. But since $|N(b) \backslash T[\ell-1]| \leq k_{1}$, and as $|N(b)| \geq 0.1 \theta k \geq 100 k_{1}$, the only possible reason why $b \notin T_{3}[\ell]$ is that $b \in T_{3}[\ell-1]$ (cf. the definition of $T_{3}[\ell]$ ). As $N(b) \not \subset T_{2}[\ell]$, T2e implies

$$
|N(b) \cap H[\ell-1]| \geq 3|N(b)| / 4 .
$$


Let $J=N(b) \cap H[\ell-1]$. Since $b \in T_{3}[\ell-1]$, we have $\ell \geq 2$ and $|N(b) \backslash T[\ell-2]| \leq$ $k_{1}$. Therefore, Corollary 3.9 implies that $\left|\Delta_{y \rightarrow b}\right| \leq 0.01$ for all $y \in J$. Thus, for all $x \in N(b)$ we have

$$
\begin{aligned}
\mu_{b \rightarrow x}^{[\ell-1]}(-\operatorname{sign}(x, b)) & =1-\prod_{y \in N(b) \backslash\{x\}} \mu_{y \rightarrow b}^{[\ell-1]}(-\operatorname{sign}(y, b)) \\
& \geq 1-(0.501)^{|J|-1} \stackrel{(54)}{\geq} 1-(0.501)^{3|N(b)| / 4-1} \geq 1-(0.501)^{0.07 k \theta},
\end{aligned}
$$

Consequently, $\mu_{b \rightarrow x}^{[\ell-1]}(-1)>0$ and

$$
\left|\frac{\mu_{b \rightarrow x}^{[\ell-1]}(1)}{\mu_{b \rightarrow x}^{[\ell-1]}(-1)}-1\right| \leq 2 \cdot(0.501)^{0.07 k \theta} \leq \exp (-\theta k / 100) \leq \exp \left(-k_{1}\right) .
$$

Thus, we have established the assertion in either case.

Proof of Proposition 3.5. We proceed by induction on $\ell$. Since $B[0]=\emptyset$, the assertion is trivial for $\ell=0$. Thus, assume that $\ell \geq 0$ and that $B[\ell] \subset T[\ell]$. Let $x \in V_{t} \backslash T[\ell+1]$. We will prove that $x \notin B[\ell+1]$. Corollary 3.10 implies that

$$
\mu_{a \rightarrow x}^{[\ell]}(-1)>0 \text { and }\left|\frac{\mu_{a \rightarrow x}^{[\ell]}(1)}{\mu_{a \rightarrow x}^{[\ell]}(-1)}-1\right| \leq \exp \left(-k_{1} / 2\right) \quad \text { for all } a \in N(x) .
$$

We claim

$$
\left|P_{>1}^{[\ell+1]}(x \rightarrow a)-1\right| \leq \delta / 100 \text { for all } a \in N(x) .
$$

To establish Equation (56), we consider two cases.

Case 1: $x \notin N\left(T_{3}[\ell]\right)$. Let $\mathcal{T}=N_{>1}^{[\ell+1]}(x \rightarrow a)$ be the set of all clauses $b$ that contribute to the product $P_{>1}^{[\ell+1]}(x \rightarrow a)$. Since $x \notin N\left(T_{3}[\ell] \cup T_{3}[\ell+1]\right)$, none of the clauses $b \in \mathcal{T}$ features more than $|N(b)|-k_{1}$ variables from $T[\ell]$ (just by the definition of $\left.T_{3}[\ell+1]\right)$. Furthermore, because $x \notin T_{2}[\ell+1]$, T2c is not satisfied and thus we obtain the bound

$$
\sum_{b \in \mathcal{T}} 2^{|N(b) \cap T[\ell] \backslash\{x\}|-|N(b)|} \leq \sum_{b \in N_{>1}(x, T[\ell])} 2^{|N(b) \cap B[\ell] \backslash\{x\}|-|N(b)|} \leq \delta /(\theta k) \leq \delta / 10^{4} .
$$

Since $x \notin T[\ell+1]$, T2a ensures that $|N(b)| \leq 10 \theta k$ for all $b \in \mathcal{T}$. Therefore, Equation (56) follows from Equation (57) and Corollary 3.8.

Case 2: $x \in N\left(T_{3}[\ell]\right)$. Let $\mathcal{T}=N_{>1}^{[\ell+1]}(x \rightarrow a) \backslash T_{3}[\ell]$ be the set of all clauses $b$ that occur in the product $P_{>1}^{[\ell+1]}(x \rightarrow a)$, apart from the ones in $T_{3}[\ell]$. Since $x \notin T_{2}[\ell+1] \cup$ $N\left(T_{3}[\ell+1]\right)$, this set $\mathcal{T}$ also satisfies Equation (57). Thus, Corollary 3.8 yields

$$
\left|\ln \prod_{b \in \mathcal{T}} \frac{\mu_{b \rightarrow x}^{[\ell]}(1)}{\mu_{b \rightarrow x}^{[\ell]}(-1)}\right| \leq \delta / 10^{3} .
$$

Let $\mathcal{T}^{\prime}=N_{>1}^{[\ell+1]}(x \rightarrow a) \cap T_{3}[\ell]$. As condition T2d ensures that $\left|\mathcal{T}^{\prime}\right| \leq\left|N(x) \cap T_{3}[\ell]\right| \leq$ 100, Equation (55) implies

$$
\left|\ln \prod_{b \in \mathcal{T}^{\prime}} \frac{\mu_{b \rightarrow x}^{[\ell]}(1)}{\mu_{b \rightarrow x}^{[\ell]}(-1)}\right| \leq 2\left|\mathcal{T}^{\prime}\right| \exp \left(-k_{1} / 2\right) \leq \delta / 1000 .
$$

Since $N_{>1}^{[\ell+1]}(x \rightarrow a)=\mathcal{T} \cup \mathcal{T}^{\prime}$, Equations (58) and (59) yield $\left|1-P_{>1}^{[\ell+1]}(x \rightarrow a)\right| \leq$ $\delta / 100$.

Thus, we have established Equation (56) in either case. 
If $x \notin T_{1}[\ell+1] \subset T[\ell+1]$ and $a \in N(x)$, then $\left|P_{\leq 1}^{[\ell+1]}(x \rightarrow a)-1\right| \leq \delta / 100$. Hence, Equation (55) implies that, for all $x \notin T[\ell+1]$ and all $a \in N(x)$, we have $\mu_{x \rightarrow a}^{[\ell+1]}(-1)>0$. Thus,

$$
\left|1-\frac{\mu_{x \rightarrow a}^{[\ell+1]}(1)}{\mu_{x \rightarrow a}^{[\ell+1]}(-1)}\right|=\left|1-P_{\leq 1}^{[\ell+1]}(x \rightarrow a) \cdot P_{>1}^{[\ell+1]}(x \rightarrow a)\right| \leq \delta / 50 \quad[\text { by }(56)] .
$$

Consequently, $\left|\Delta_{x \rightarrow a}^{[\ell+1]}\right|<0.1 \delta$, and thus $x \notin B[\ell+1]$.

\subsection{Proof of Proposition 3.6}

We are going to proceed by induction on $\ell$. We begin by bounding the sizes of the sets $T_{2}, T_{3}$.

Lemma 3.11. Assume that $\left|T_{1}[\ell] \cup T_{2}[\ell]\right| \leq \delta \theta n / 3$ and $\left|N\left(T_{3}[\ell]\right)\right| \leq \delta \theta n / 2$. Then $\left|N\left(T_{3}[\ell+1]\right)\right| \leq \delta \theta n / 2$.

Proof. By construction, we have $T_{3}[\ell] \cap T_{3}[\ell+1]=\varnothing$ (cf. Equation (42)). Furthermore, also by construction $N\left(T_{3}[\ell]\right) \subset T[\ell]$, and each clause in $T_{3}[\ell+1]$ has at least a 0.99 -fraction of its variables in $T[\ell]$. Thus, $|N(b) \cap T[\ell]| \geq 0.99|N(b)|$ for all $b \in T_{3}[\ell] \cup T_{3}[\ell+1]$. Hence, $\mathbf{Q 3}$ yields

$$
\begin{aligned}
\left|N\left(T_{3}[\ell+1]\right)\right|+\left|N\left(T_{3}[\ell]\right)\right| & \leq \sum_{b \in T_{3}[\ell] \cup T_{3}[\ell+1]}|N(b)| \\
& \leq \frac{1.01}{0.99}|T[\ell]| \leq 1.03\left(\left|T_{1}[\ell]\right|+\left|T_{2}[\ell]\right|+\left|N\left(T_{3}[\ell]\right)\right|\right) .
\end{aligned}
$$

Hence, $\left|N\left(T_{3}[\ell+1]\right)\right| \leq 1.03\left(\left|T_{1}[\ell]\right|+\left|T_{2}[\ell]\right|\right)+0.03\left|N\left(T_{3}[\ell]\right)\right| \leq \theta \delta n / 2$.

Lemma 3.12. Assume that $\left|T_{1}[\ell] \cup T_{2}[\ell]\right| \leq \delta \theta n / 3$ and $\left|N\left(T_{3}[\ell]\right)\right| \leq \delta \theta n / 2$. Moreover, suppose that $|T[\ell-1]| \leq \delta \theta n$. Then $\left|T_{2}[\ell+1]\right| \leq \delta \theta n / 6$.

Proof. Conditions $\mathbf{Q 0}$ and $\mathbf{Q 1}$ readily imply that the number of variables that satisfy either T2a or T2b is $\leq 0.001 \theta \delta n$. Moreover, we apply $\mathbf{Q 2}$ to the set $T[\ell]$ of size

$$
|T[\ell]| \leq\left|T_{1}[\ell] \cup T_{2}[\ell]\right|+\left|N\left(T_{3}[\ell]\right)\right| \leq 0.9 \delta \theta n
$$

to conclude that the number of variables satisfying T2c is $\leq 0.001 \theta \delta n$ as well.

To bound the number of variables that satisfy T2d, consider the subgraph of the factor graph induced on $T_{3}[\ell] \cup N\left(T_{3}[\ell]\right)$. For each $x \in N\left(T_{3}[\ell]\right)$, let $D_{x}$ be the number of neighbors of $x$ in $T_{3}[\ell]$. Let $v$ be the number of $x \in V_{t}$ so that $D_{x} \geq 100$. Then $\mathbf{Q 3}$ yields

$$
100 v \leq \sum_{x \in N\left(T_{3}[\ell]\right)} D_{x}=\sum_{a \in T_{3}[\ell]}|N(a)| \leq 1.01|T[\ell]|+10^{-4} \theta \delta n \leq \theta \delta n,
$$

as $N(b) \subset T[\ell]$ if $b \in T_{3}[\ell]$. Hence, there are at most $v \leq 0.01 \theta \delta n$ variables that satisfy T2d. In summary, we have shown that

$$
\mid\{x \in V: x \text { satisfies one of T2a-T2d }\} \mid \leq 0.015 \theta \delta n \text {. }
$$

To deal with T2e, observe that if a clause $a$ has at least $|N(a)| / 4$ variables that are not harmless, then one of the following statements is true.

i. $a$ contains at least $|N(a)| / 20$ variables $x$ that violate either H1, H2, or H4.

ii. $a$ contains at least $|N(a)| / 5$ variables $x$ that violate condition $\mathbf{H 3}$.

Let $\mathcal{C}_{1}$ be the set of clauses $a$ for which (i) holds and let $\mathcal{C}_{2}$ be the set of clauses satisfying (ii), so that the number of variables satisfying T2e is bounded by $\sum_{a \in \mathcal{C}_{1} \cup \mathcal{C}_{2}}|N(a)|$. 
To bound $\sum_{a \in \mathcal{C}_{1}}|N(a)|$, let $Q$ be the set of all variables $x$ that violate either $\mathbf{H 1}, \mathbf{H 2}$, or H4. Then conditions $\mathbf{Q 1}$ and $\mathbf{Q 2}$ entail that $|Q| \leq 3 \cdot 10^{-4} \theta \delta n$ (because we are assuming $|T(\ell-1)| \leq \theta \delta n)$. Therefore, condition $\mathbf{Q 3}$ implies that

$$
\sum_{a \in \mathcal{C}_{1}}|N(a)| \leq 21|Q|+10^{-4} \delta \theta n \leq 0.0064 \delta \theta n .
$$

To deal with $\mathcal{C}_{2}$, let $\mathcal{B}^{\prime}$ be the set of all clauses $b$ such that $|N(b)| \geq 100 k_{1}$ but $|N(b) \backslash T[\ell]| \leq k_{1}$. Since we know from Equation (60) that $|T[\ell]| \leq \delta \theta n$, condition Q3 applied to $T[\ell]$ implies

$$
\left|N\left(\mathcal{B}^{\prime}\right)\right| \leq \sum_{b \in \mathcal{B}^{\prime}}|N(b)| \leq 1.03|T[\ell]|+10^{-4} \delta \theta n \leq 1.0301 \delta \theta n .
$$

In addition, let $\mathcal{B}^{\prime \prime}$ be the set of all clauses of length less than $100 k_{1}$. Since $100 k_{1}=$ $100 \sqrt{c} \theta k \leq 0.1 \theta k$ by our choice of $c, \mathbf{Q} 1$ implies that $\left|N\left(\mathcal{B}^{\prime \prime}\right)\right| \leq 10^{-4} \delta \theta n$. Hence, Equation (63) shows that $\mathcal{B}=\mathcal{B}^{\prime} \cup \mathcal{B}^{\prime \prime}$ satisfies

$$
|N(\mathcal{B})| \leq 1.0302 \delta \theta n
$$

Furthermore, let $\mathcal{U}$ be the set of all clauses $a$ such that $N(a) \subset N(\mathcal{B})$. Let $U$ be the set of variables $x \in N(\mathcal{B})$ that occur in at least two clauses from $\mathcal{U}$. Then by $\mathbf{Q 3}$

$$
|U|+|N(\mathcal{B})| \leq \sum_{a \in \mathcal{U}}|N(a)| \leq 1.01|N(\mathcal{B})|+10^{-4} \delta \theta n
$$

whence $|U| \leq 0.01|N(\mathcal{B})|+10^{-4} \delta \theta n \leq 0.02 \delta \theta n$ due to Equation (64). Since $\mathcal{B} \subset \mathcal{U}$, the set $U$ contains all variables that occur in at least two clauses from $\mathcal{B}$ (i.e., all variables that violate condition H3). Therefore, any $a \in \mathcal{C}_{2}$ contains at least $|N(a)| / 5$ variables from $U$. Applying $\mathbf{Q 3}$ once more, we obtain

$$
\sum_{a \in \mathcal{C}_{2}}|N(a)| \leq 5.05 \cdot 0.02 \delta \theta n+10^{-4} \delta \theta n=0.1201 \delta \theta n
$$

Combining this estimate with the bound of Equation (62) on $\mathcal{C}_{1}$, we conclude that the number of variables satisfying T2e is bounded by $\sum_{a \in \mathcal{C}_{1} \cup \mathcal{C}_{2}}|N(a)| \leq 0.127 \delta \theta n$. Together with Equation (61), this yields the assertion.

In Section 3.6, we derive the following bound on $\left|T_{1}[\ell+1]\right|$ :

Proposition 3.13. If $|T[\ell]| \leq \delta \theta n$, then $\left|T_{1}[\ell+1] \backslash T_{2}[\ell+1]\right| \leq \delta \theta n / 6$.

Proof Proposition 3.6. We are going to show that

$$
\left|T_{1}[\ell] \cup T_{2}[\ell]\right| \leq \delta \theta n / 3 \text { and }\left|N\left(T_{3}[\ell]\right)\right| \leq \delta \theta n / 2
$$

for all $\ell \geq 0$. This implies that $|T[\ell]| \leq \delta \theta n$ for all $\ell \geq 0$, as desired.

To prove Equation (65), we proceed by induction on $\ell$. The bounds for $\ell=0$ are immediate from $\mathbf{Q 0}$ and $\mathbf{Q 1}$. Now assume that Equation (65) holds for all $l \leq \ell$. Then Lemma 3.11 shows that $\left|N\left(T_{3}[\ell+1]\right)\right| \leq \delta \theta n / 2$. Moreover, Lemma 3.12 applies (with the convention that $T[-1]=T[0])$, giving $\left|T_{2}[\ell+1]\right| \leq \delta \theta n / 6$. Finally, Proposition 3.13 shows $\left|T_{1}[\ell+1] \backslash T_{2}[\ell+1]\right| \leq \delta \theta n / 6$, whence $\left|T_{1}[\ell+1] \cup T_{2}[\ell+1]\right| \leq \delta \theta n / 3$. 


\subsection{Proof of Proposition $\mathbf{3 . 1 3}$}

Throughout this section, we assume that $|T[\ell]| \leq \delta \theta n$.

For a variable $x \in V_{t}$ and $a \in N(x)$ we let

$$
\begin{aligned}
\sigma_{x \rightarrow a}^{[\ell+1]} & =\sum_{b \in N_{\leq 1}^{[\ell+1]}(x \rightarrow a)} 2^{1-|N(b)|} \operatorname{sign}(x, b), \\
\xi_{x \rightarrow a}^{[\ell+1]} & =\sum_{b \in N_{\leq 1}^{[\ell+1]}(x \rightarrow a)} \sum_{y \in N(b) \backslash\{x\}} 2^{-|N(b)|} \operatorname{sign}(x, b) \operatorname{sign}(y, b) \Delta_{y \rightarrow b}^{[\ell]}, \text { and } \\
L_{x \rightarrow a}^{[\ell+1]} & =\sigma_{x \rightarrow a}^{[\ell+1]}+\xi_{x \rightarrow a}^{[\ell+1]} .
\end{aligned}
$$

In Section 3.7, we are going to establish the following:

Proposition 3.14. For any variable $x \notin T_{2}[\ell+1]$ and any clause $a \in N(x)$, we have

$$
\left|L_{x \rightarrow a}^{[\ell+1]}+\ln P_{\leq 1}^{[\ell+1]}(x \rightarrow a)\right| \leq 10^{-3} \delta .
$$

Lemma 3.15. For all but at most $10^{-4} \delta \theta$ n variables $x \in V \backslash T_{2}[\ell+1]$, we have

$$
\max _{a \in N(x)}\left|\sigma_{x \rightarrow a}^{[\ell+1]}\right| \leq 0.003 \delta .
$$
have

Proof. Applying $\mathbf{Q} 2$ to $T[\ell]$, we find that for all but $10^{-4} \delta \theta n$ variables $x \in V_{t}$ we

$$
\left|\sum_{b \in N_{\leq 1}(x, T[\ell])} 2^{1-|N(b)|} \operatorname{sign}(x, b)\right| \leq 2 \cdot 10^{-3} \delta .
$$

Assume that $x$ satisfies Equation (66) and that $x \notin T_{2}[\ell+1]$. Let $a \in N(x)$. Since $N_{\leq 1}^{[\ell+1]}(x \rightarrow a)=N_{\leq 1}(x, T[\ell]) \backslash\{a\}$, we obtain

$$
\begin{aligned}
\left|\sigma_{x \rightarrow a}^{[\ell+1]}\right| & \leq\left|\sum_{b \in N_{\leq 1}(x, T[\ell])} 2^{1-|N(b)|} \operatorname{sign}(x, b)\right|+2^{1-|N(a)|} \leq 2 \cdot 10^{-3} \delta+2^{1-|N(a)|} \\
& \leq 2 \cdot 10^{-3} \delta+\exp (-0.1 \theta k) \leq 0.003 \delta \quad[\text { as }|N(a)| \geq 0.1 \theta k \text { due to T2a }],
\end{aligned}
$$

as desired.

Lemma 3.16. Let $x$ be a variable and let $b_{1}, b_{2} \in N(x)$ be such that $\left|N\left(b_{i}\right) \cap T[\ell]\right| \leq 2$ and $\left|N\left(b_{i}\right)\right| \geq 0.1 \theta k$ for $i=1,2$. Then, $\left|\Delta_{x \rightarrow b_{1}}^{[\ell]}-\Delta_{x \rightarrow b_{2}}^{[\ell]}\right| \leq \delta^{3}$.

Proof. By Proposition 3.5, we have $B[\ell-1] \subset T[\ell-1]$. Furthermore, our assumptions ensure that $N\left(b_{i}\right) \backslash T[\ell] \neq \emptyset$. Hence, Corollary 3.10 yields

$$
\mu_{b_{i} \rightarrow x}^{[\ell-1]}(-1)>0 \text { and }\left|\frac{\mu_{b_{i} \rightarrow x}^{[\ell-1]}(1)}{\mu_{b_{i} \rightarrow x}^{[\ell-1]}(-1)}-1\right| \leq \exp \left(-k_{1} / 2\right) \leq \delta^{6}
$$

for $i=1,2$. There are three cases.

Case 1: There is $c \in N(x) \backslash\left\{b_{1}, b_{2}\right\}$ such that $\mu_{c \rightarrow x}^{[\ell-1]}(1)=0$ Then Equation (28) shows that

$$
\mu_{x \rightarrow b_{1}}^{[\ell]}(1)=\mu_{x \rightarrow b_{2}}^{[\ell]}(1)=0 .
$$

Thus, $\Delta_{x \rightarrow b_{1}}^{[\ell]}=\Delta_{x \rightarrow b_{2}}^{[\ell]}=-1 / 2$. 
Case 2: There is $c \in N(x) \backslash\left\{b_{1}, b_{2}\right\}$ such that $\mu_{c \rightarrow x}^{[\ell-1]}(-1)=0$ Similarly as in Case 1, Equation (28) implies $\mu_{x \rightarrow b_{i}}^{[\ell]}(-1)=0$ for $i=1$, 2. Since $\mu_{x \rightarrow b_{i}}^{[\ell]}(-1)+\mu_{x \rightarrow b_{i}}^{[\ell]}(1)=1$, we thus obtain $\Delta_{x \rightarrow b_{1}}^{[\ell]}=\Delta_{x \rightarrow b_{2}}^{[\ell]}=1 / 2$.

Case 3: For all $c \in N(x) \backslash\left\{b_{1}, b_{2}\right\}$, we have $0<\mu_{c \rightarrow x}^{[\ell-1]}(1)<1$ Then, Equation (28) yields $0<\mu_{x \rightarrow b_{i}}^{[\ell]}(-1)<1$ for $i=1,2$. Therefore, we can define

$$
q_{i}=\frac{\mu_{x \rightarrow b_{i}}^{[\ell]}(1)}{\mu_{x \rightarrow b_{i}}^{[\ell]}(-1)}=\frac{\prod_{b \in N(x) \backslash\left\{b_{i}\right\}} \mu_{b \rightarrow x}^{[\ell-1]}(1)}{\prod_{b \in N(x) \backslash\left\{b_{i}\right\}} \mu_{b \rightarrow x}^{[\ell-1]}(-1)}>0 .
$$

Unraveling Equation (28), we see that

$$
\begin{aligned}
\mu_{x \rightarrow b_{i}}^{[\ell]}(1) & =\frac{\prod_{b \in N(x) \backslash\left\{b_{i}\right\}} \mu_{b \rightarrow x}^{[\ell-1]}(1)}{\prod_{b \in N(x) \backslash\left\{b_{i}\right\}} \mu_{b \rightarrow x}^{[\ell-1]}(1)+\prod_{b \in N(x) \backslash\left\{b_{i}\right\}} \mu_{b \rightarrow x}^{[\ell-1]}(-1)} \\
& =\frac{\prod_{b \in N(x) \backslash\left\{b_{i}\right\}} \mu_{b \rightarrow x}^{[\ell-1]}(1)}{\left(1+1 / q_{i}\right) \prod_{b \in N(x) \backslash\left\{b_{i}\right\}} \mu_{b \rightarrow x}^{[\ell-1]}(1)}=\frac{q_{i}}{q_{i}+1} .
\end{aligned}
$$

Hence,

$$
\begin{aligned}
\left|\Delta_{x \rightarrow b_{1}}^{[\ell]}-\Delta_{x \rightarrow b_{2}}^{[\ell]}\right| & =\left|\mu_{x \rightarrow b_{1}}^{[\ell]}(1)-\mu_{x \rightarrow b_{2}}^{[\ell]}(1)\right| \\
& =\left|\frac{q_{1}-q_{2}}{\left(1+q_{1}\right)\left(1+q_{2}\right)}\right| \quad[\text { by (69)] } \\
& \left.=\left|\frac{1-q_{2} / q_{1}}{\left(1+1 / q_{1}\right)\left(1 / q_{1}+q_{2} / q_{1}\right)}\right| \leq \frac{q_{1}}{q_{2}}\left|1-\frac{q_{2}}{q_{1}}\right| \quad \text { [as } q_{1}, q_{2}>0\right] .
\end{aligned}
$$

Furthermore, by the definition in Equation (68) of $q_{1}, q_{2}$, we have

$$
\frac{q_{2}}{q_{1}}=\frac{\mu_{b_{1} \rightarrow x}^{[\ell-1]}(1)}{\mu_{b_{1} \rightarrow x}^{[\ell-1]}(-1)} \cdot \frac{\mu_{b_{2} \rightarrow x}^{[\ell-1]}(-1)}{\mu_{b_{2} \rightarrow x}^{[\ell-1]}(1)} .
$$

Hence, Equation (67) yields $\left|1-\frac{q_{2}}{q_{1}}\right| \leq \delta^{5}$, and thus the desired bound on $\left|\Delta_{x \rightarrow b_{1}}^{[\ell]}-\Delta_{x \rightarrow b_{2}}^{[\ell]}\right|$ follows from Equation (70).

Hence, we have established the desired bound in all cases.

LEMMA 3.17. For all but at most $0.1 \delta \theta n$ variables $x \notin T_{2}[\ell+1]$ we have

$$
\max _{a \in N(x)}\left|\xi_{x \rightarrow a}^{[\ell+1]}\right| \leq 0.001 \delta \text {. }
$$

Proof. For a variable $y$ let $\mathcal{N}(y)$ be the set of all clauses $b \in N(y)$ such that $b \in$ $N_{\leq 1}(x, T[\ell])$ for some variable $x \in V_{t}$. If $\mathcal{N}(y)=\emptyset$ we define $\Delta_{y}=0$; otherwise select $a_{y} \in \mathcal{N}(y)$ arbitrarily and set $\Delta_{y}=\Delta_{y \rightarrow a_{y}}^{[\ell]}$. Thus, we obtain a vector $\Delta=\left(\Delta_{y}\right)_{y \in V}$ with norm $\|\Delta\|_{\infty} \leq \frac{1}{2}$. Let $\Xi=\left(\xi_{x}\right)_{x \in V_{t}}=\Lambda_{T[\ell]} \Delta$, where $\Lambda_{T[\ell]}$ is the linear operator from condition $\mathbf{Q} 4$ in Definition 3.2. That is, for any $x \in V_{t}$ we have

$$
\xi_{x}=\sum_{b \in N_{\leq 1}(x, T[\ell])} \sum_{y \in N(b) \backslash\{x\}} 2^{-|N(b)|} \operatorname{sign}(x, b) \operatorname{sign}(y, b) \Delta_{y} .
$$

Because $|T[\ell]| \leq \delta \theta n$, condition $\mathbf{Q 4}$ ensures that $\left\|\Lambda_{T[\ell]}\right\|_{\square} \leq \delta^{4} \theta n$. Consequently,

$$
\|\Xi\|_{1}=\left\|\Lambda_{T[\ell]} \Delta\right\|_{1} \leq\left\|\Lambda_{T[\ell]}\right\|_{\square}\|\Delta\|_{\infty} \leq \delta^{4} \theta n .
$$


Since $\|\Xi\|_{1}=\sum_{x \in V_{t}}\left|\xi_{x}\right|$, Equation (71) implies that

$$
\left|\left\{x \in V_{t}:\left|\xi_{x}\right|>\delta^{2}\right\}\right| \leq \delta^{2} \theta n .
$$

To deduce the lemma from Equation (72), suppose that $x \notin T_{2}[\ell+1]$. Then,

$$
\begin{aligned}
\max _{a \in N(x)}\left|\xi_{x}-\xi_{x \rightarrow a}^{[\ell+1]}\right|= & \max _{a \in N(x)} \mid \mathbf{1}_{a \in N_{\leq 1}(x, T[\ell])} \cdot \sum_{y \in N(a) \backslash\{x\}} 2^{-|N(b)|} \operatorname{sign}(x, b) \operatorname{sign}(y, b) \Delta_{y} \\
& +\sum_{b \in N_{\leq 1}^{[\ell+1]}(x \rightarrow a)} \sum_{y \in N(b) \backslash\{x\}} 2^{-|N(b)|} \operatorname{sign}(x, b) \operatorname{sign}(y, b)\left(\Delta_{y}-\Delta_{y \rightarrow b}^{[\ell]}\right) \mid \\
\leq & \sum_{y \in N(a) \backslash\{x\}} 2^{-|N(a)|}\left|\Delta_{y}\right|+\sum_{b \in N_{\leq 1}^{[\ell+1]}(x \rightarrow a)} \sum_{y \in N(b) \backslash\{x\}} 2^{-|N(b)|}\left|\Delta_{y \rightarrow b}^{[\ell]}-\Delta_{y}\right| \\
\leq & |N(a)| 2^{-|N(a)|}+\sum_{b \in N_{\leq 1}^{[\ell+1]}(x \rightarrow a)} \sum_{y \in N(b) \backslash\{x\}} 2^{-|N(b)|}\left|\Delta_{y \rightarrow b}^{[\ell]}-\Delta_{y}\right| .
\end{aligned}
$$

Furthermore, if $b \in N_{\leq 1}^{[\ell+1]}(x \rightarrow a)$, then $0.1 \theta k \leq|N(b)| \leq 10 \theta k$ and $|N(b) \cap T[\ell]| \leq 2$ by Equations (39) and (40). Similarly, if $y \in N(b)$, then $\mathcal{N}(y) \neq \varnothing$ (because $b \in \mathcal{N}(y)$ ) and the chosen clause $a_{y} \in \mathcal{N}(y)$ satisfies $0.1 \theta k \leq\left|N\left(a_{y}\right)\right| \leq 10 \theta k$ and $\left|N\left(a_{y}\right) \cap T[\ell]\right| \leq 2$ because $a_{y} \in N_{\leq 1}\left(x^{\prime}, T[\ell]\right)$ for some variable $x^{\prime}$. Therefore, Lemma 3.16 implies that

$$
\left|\Delta_{y \rightarrow b}^{[\ell]}-\Delta_{y}\right|=\left|\Delta_{y \rightarrow b}^{[\ell]}-\Delta_{y \rightarrow a_{y}}^{[\ell]}\right| \leq \delta^{3} \quad \text { for all } b \in N_{\leq 1}^{[\ell+1]}(x \rightarrow a), y \in N(b) .
$$

Combining Equations (73) and (74), we obtain

$$
\begin{aligned}
& \max _{a \in N(x)}\left|2 \xi_{x}-\xi_{x \rightarrow a}^{[\ell+1]}\right| \leq|N(a)| 2^{-|N(a)|}+\delta^{3} \sum_{b \in N(x)}|N(b)| 2^{1-|N(b)|} \\
& \leq 10 k \theta 2^{-0.1 k \theta}+10 \delta^{3} k \theta \sum_{b \in N(x)} 2^{1-|N(b)|} \quad \text { [as } 0.1 k \theta \leq|N(a)| \leq 10 k \theta \text { by T2a] } \\
& \leq 10^{-4} \delta \quad \text { [by } \mathbf{T} 2 \mathbf{b} \text { ]. }
\end{aligned}
$$

If $x \notin T_{2}[\ell+1]$ is such that $\left|\xi_{x}\right| \leq \delta^{2}$, then Equation (75) implies that $\left|\xi_{x \rightarrow a}\right| \leq 2 \cdot 10^{-4} \delta$ for any $a \in N(x)$. Therefore, the assertion follows from Equation (72).

Proof of Proposition 3.13. Let $S$ be the set of all variables $x \notin T_{2}[\ell+1]$ such that $\max _{a \in N(x)}\left|\sigma_{x \rightarrow a}^{[\ell+1]}\right| \leq 0.003 \delta$ and $\max _{a \in N(x)}\left|\xi_{x \rightarrow a}^{[\ell+1]}\right| \leq 0.001 \delta$. Then, Proposition 3.14 entails that for any $\bar{x} \in S$ and $a \in N(x)$

$$
\left|\ln P_{\leq 1}^{[\ell+1]}(x \rightarrow a)\right| \leq\left|L_{x \rightarrow a}^{[\ell+1]}\right|+10^{-3} \delta \leq\left|\sigma_{x \rightarrow a}^{[\ell+1]}\right|+\left|\xi_{x \rightarrow a}^{[\ell+1]}\right|+10^{-3} \delta \leq 0.005 \delta .
$$

Hence, $\left|P_{\leq 1}^{[\ell+1]}(x \rightarrow a)-1\right| \leq 0.01 \delta$ for all $x \in S, a \in N(x)$, and therefore

$$
\left.T_{1}[\ell+1] \backslash T_{2}[\ell+1] \subset V_{t} \backslash\left(S \cup T_{2}[\ell+1]\right)\right) .
$$

Finally, Lemmas 3.15 and 3.17 imply $\left|T_{1}[\ell+1] \backslash T_{2}[\ell+1]\right| \leq\left|V_{t} \backslash\left(S \cup T_{2}[\ell+1]\right)\right| \leq$ $\delta \theta n / 6$.

\subsection{Proof of Proposition 3.14}

We begin by approximating $\ln \left(\mu_{b \rightarrow x}^{[\ell]}(1) / \mu_{b \rightarrow x}^{[\ell]}(-1)\right)$ by a linear function. In this section, we let $O_{\rho}(\cdot)$ denote an asymptotic bound that holds in the limit of large $\rho$. That is, $f(\rho)=O(g(\rho))$ if there exist $C>0, \rho_{*}>0$ such that $|f(\rho)| \leq C|g(\rho)|$ for $\rho>\rho_{*}$. 
Lemma 3.18. Let $x \in V_{t}, a \in N(x)$, and $b \in N_{\leq 1}^{[\ell+1]}(x \rightarrow a)$. Then, $\mu_{b \rightarrow x}^{[\ell]}(-1)>0$ and

$$
\begin{aligned}
\ln \left(\frac{\mu_{b \rightarrow x}^{[\ell]}(1)}{\mu_{b \rightarrow x}^{[\ell]}(-1)}\right)= & 2^{1-|N(b)|}\left[\operatorname{sign}(x, b)+2 \sum_{y \in N(b) \backslash\{x\}} \operatorname{sign}(x, b) \operatorname{sign}(y, b) \Delta_{y \rightarrow b}^{[\ell]}\right] \\
& +\frac{(\theta k \delta+|N(b) \cap T[\ell] \backslash\{x\}|)}{2^{|N(b)|} \cdot O_{\rho}(k \theta \delta) .}
\end{aligned}
$$

Proof. The definition of the set $N_{\leq 1}^{[\ell+1]}(x \rightarrow a)$ ensures that, for all $b \in N_{\leq 1}^{[\ell+1]}(x \rightarrow a)$, we have $|N(b) \cap T[\ell]| \leq 2$, while $|N(b)| \geq 0.1 \theta k$. Therefore, Lemma 3.7 shows that $\left|1-\mu_{b \rightarrow x}^{[\ell]}(-1)\right| \leq \delta^{2}$ (recall from Proposition 3.5 that $B[\ell] \subset T[\ell]$ ). Furthermore, $b$ is not redundant, and thus not a tautology, because otherwise $N(b) \subset T_{2}[\ell]$ due to T2a.

Let $s=\operatorname{sign}(x, b)$. Then, $\mu_{b \rightarrow x}^{[\ell]}(s)=1$ and thus the definition (2) of the messages $\mu_{b \rightarrow x}^{[\ell]}( \pm 1)$ yields

$$
\begin{aligned}
\frac{\mu_{b \rightarrow x}^{[\ell]}(-s)}{\mu_{b \rightarrow x}^{[\ell]}(s)} & =\mu_{b \rightarrow x}^{[\ell]}(-s)=1-\prod_{y \in N(b) \backslash\{x\}} \mu_{y \rightarrow b}^{[\ell]}(-\operatorname{sign}(y, b)) \\
& =1-\prod_{y \in N(b) \backslash\{x\}} \frac{1}{2}-\operatorname{sign}(y, b) \Delta_{y \rightarrow b}^{[\ell]} \\
& =1-2^{1-|N(b)|} \prod_{y \in N(b) \backslash\{x\}} 1-2 \operatorname{sign}(y, b) \Delta_{y \rightarrow b}^{[\ell]} .
\end{aligned}
$$

Let $\Gamma=N(b) \backslash(T[\ell] \cup\{x\})$. Because Proposition 3.5 shows that $T[\ell] \supset B[\ell]$ contains all biased variables, we have $\left|\Delta_{y \rightarrow b}^{[\ell]}\right| \leq \delta$ for all $y \in \Gamma$. Therefore, we can use the approximation $|\ln (1-z)+z| \leq z^{2}$ for $|z| \leq \frac{1}{2}$ to obtain

$$
\begin{aligned}
\left|\left[\ln \prod_{y \in \Gamma} 1-2 \operatorname{sign}(y, b) \Delta_{y \rightarrow b}^{[\ell]}\right]+\sum_{y \in \Gamma} 2 \operatorname{sign}(y, b) \Delta_{y \rightarrow b}^{[\ell]}\right| \\
=\left|\sum_{y \in \Gamma} \ln \left(1-2 \operatorname{sign}(y, b) \Delta_{y \rightarrow b}^{[\ell]}\right)+2 \operatorname{sign}(y, b) \Delta_{y \rightarrow b}^{[\ell]}\right| \\
\leq 4 \sum_{y \in \Gamma} \Delta_{y \rightarrow b}^{[\ell]}{ }^{2} \leq 40 \theta k \delta^{2} ;
\end{aligned}
$$

in the last step, we used that $|N(b)| \leq 10 \theta k$ for all $b \in N_{\leq 1}^{[\ell+1]}(x \rightarrow a)$. Furthermore, $|\Gamma| \leq|N(b)| \leq 10 \theta k$ and $\left|\Delta_{y \rightarrow b}^{[\ell]}\right| \leq \delta$ for all $y \in \Gamma$. Hence,

$$
\left|\sum_{y \in \Gamma} 2 \operatorname{sign}(y, b) \Delta_{y \rightarrow b}^{[\ell]}\right| \leq 20 \delta k \theta .
$$


Therefore, taking exponentials in Equation (78), we obtain

$$
\begin{aligned}
\prod_{y \in \Gamma} 1-2 \operatorname{sign}(y, b) \Delta_{y \rightarrow b}^{[\ell]} & =\exp \left[O_{\rho}(\theta k \delta)^{2}-\sum_{y \in \Gamma} 2 \operatorname{sign}(y, b) \Delta_{y \rightarrow b}^{[\ell]}\right] \\
& =1-\sum_{y \in \Gamma} 2 \operatorname{sign}(y, b) \Delta_{y \rightarrow b}^{[\ell]}+O_{\rho}(\theta k \delta)^{2} .
\end{aligned}
$$

Furthermore, the definition of $N_{\leq 1}^{[\ell+1]}(x \rightarrow a)$ ensures that

$$
|N(b) \backslash(\Gamma \cup\{x\})=| N(b) \cap T[\ell] \backslash\{x\} \mid \leq 1 .
$$

If there is $y_{0} \in N(b) \cap T[\ell] \backslash\{x\}$, then Equations (79) and (80) yield

$$
\begin{aligned}
\prod_{y \in N(b) \backslash\{x\}} 1-2 \operatorname{sign}(y, b) \Delta_{y \rightarrow b}^{[\ell]} & =\left(1-2 \operatorname{sign}\left(y_{0}, b\right) \Delta_{y_{0} \rightarrow b}^{[\ell]}\right) \cdot \prod_{y \in \Gamma} 1-2 \operatorname{sign}(y, b) \Delta_{y \rightarrow b}^{[\ell]} \\
& =1-2\left[\sum_{y \in N(b) \backslash\{x\}} \operatorname{sign}(y, b) \Delta_{y \rightarrow b}^{[\ell]}\right]+O_{\rho}(\theta k \delta) .
\end{aligned}
$$

Hence, in any case, we have

$$
\begin{aligned}
\prod_{y \in N(b) \backslash\{x\}} 1-2 \operatorname{sign}(y, b) \Delta_{y \rightarrow b}^{[\ell]}= & 1-2\left[\sum_{y \in N(b) \backslash\{x\}} \operatorname{sign}(y, b) \Delta_{y \rightarrow b}^{[\ell]}\right] \\
& +(\theta k \delta+|N(b) \cap T[\ell] \backslash\{x\}|) \cdot O_{\rho}(\theta k \delta) .
\end{aligned}
$$

Combining this with Equation (77) and using the approximation $|\ln (1-z)+z| \leq z^{2}$ for $|z| \leq 1 / 2$, we see that

$$
\begin{aligned}
\ln \left(\frac{\mu_{b \rightarrow x}^{[\ell]}(-s)}{\mu_{b \rightarrow x}^{[\ell]}(s)}\right)= & -2^{1-|N(b)|}\left[1-2 \sum_{y \in N(b) \backslash\{x\}} \operatorname{sign}(y, b) \Delta_{y \rightarrow b}^{[\ell]}\right] \\
& +2^{1-|N(b)|}(\theta k \delta+|N(b) \cap T[\ell] \backslash\{x\}|) \cdot O_{\rho}(\theta k \delta),
\end{aligned}
$$

whence the assertion follows.

Proof Proposition 3.14. Suppose $x \notin T_{2}[\ell+1]$. By the definition of $P_{\leq 1}^{[\ell+1]}(x \rightarrow a)$ we have

$$
\ln P_{\leq 1}^{[\ell+1]}(x \rightarrow a)=\sum_{b \in N_{\leq 1}^{[\ell+1]}(x \rightarrow a)} \ln \left(\frac{\mu_{b \rightarrow x}^{[\ell]}(1)}{\mu_{b \rightarrow x}^{[\ell]}(-1)}\right)
$$

Hence, Lemma 3.18 yields

$$
\ln P_{\leq 1}^{[\ell+1]}(x \rightarrow a)=L_{x \rightarrow a}^{[\ell+1]}+\sum_{b \in N_{\leq 1}^{[\ell+1]}(x \rightarrow a)} 2^{-|N(b)|}(\theta k \delta+|N(b) \cap T[\ell] \backslash\{x\}|) O_{\rho}(\theta k \delta) .
$$


To complete the proof, we need to estimate the second summand. Condition T2b implies

$$
\begin{aligned}
O_{\rho}(\delta \theta k)^{2} \sum_{b \in N_{\leq 1}^{[\ell+1]}(x \rightarrow a)} 2^{-|N(b)|} & \leq O_{\rho}(\delta \theta k)^{2} \sum_{b \in N(x)} 2^{-|N(b)|} \\
& \leq \frac{O_{\rho}(\delta \theta k)^{2}}{\delta(\theta k)^{5}} \leq \frac{O_{\rho}(\delta)}{(\theta k)^{3}} \leq 10^{-4} \delta .
\end{aligned}
$$

Furthermore, T2c yields

$$
\begin{aligned}
O_{\rho}(\theta k \delta) \sum_{b \in N_{\leq 1}^{[\ell+1]}(x \rightarrow a)} 2^{-|N(b)|}|N(b) \cap T[\ell] \backslash\{x\}| & \leq O_{\rho}(\theta k \delta) \sum_{b \in N_{1}(x, T[\ell])} 2^{-|N(b)|} \\
& \leq O_{\rho}(\theta k \delta) \cdot \rho(\theta k)^{5} \delta \leq 10^{-4} \delta .
\end{aligned}
$$

Finally, the assertion follows by plugging Equations (82) and (83) into Equation (81).

\subsection{Completing the Proof of Theorem 3.4}

We are going to show that $\left|\mu_{x}\left(\Phi_{t}, \omega\right)-\frac{1}{2}\right| \leq \delta=\delta_{t}$ for all $x \in V_{t} \backslash T[\omega+1]$. This will imply Theorem 3.4 because $|T[\omega+1]| \leq \delta_{t}(n-t)$ by Proposition 3.6.

Thus, let $x \in V_{t} \backslash T[\omega+1]$. Corollary 3.10 shows that $\mu_{b \rightarrow x}^{[\omega]}(\zeta)>0$ for $\zeta= \pm 1$. Hence,

$$
P(\zeta)=\prod_{b \in N(x)} \mu_{b \rightarrow x}^{[\omega]}(\zeta)>0 \quad \text { for } \zeta= \pm 1
$$

Recall from Equation (4) that

$$
\mu_{x}\left(\Phi_{t}, \omega\right)=\frac{P(1)}{P(-1)+P(1)} .
$$

If $N(x)=\emptyset$, then trivially $P(-1)=P(1)=1$ and thus $\mu_{x}\left(\Phi_{t-1}, \omega\right)=\frac{1}{2}$. Thus, assume that $N(x) \neq \varnothing$ and pick an arbitrary $a \in N(x)$. Since $x \notin T[\omega+1] \supset B[\omega+1]$ (by Proposition 3.5), we have

$$
\left|\mu_{x \rightarrow a}^{[\omega+1]}(\zeta)-\frac{1}{2}\right|=\left|\Delta_{x \rightarrow a}^{[\omega+1]}\right| \leq 0.1 \delta \quad \text { for } \zeta= \pm 1 .
$$

Therefore,

$$
\begin{aligned}
& \ln \frac{\mu_{x \rightarrow a}^{[\omega+1]}(-1)}{\mu_{x \rightarrow a}^{[\omega+1]}(1)} \leq \ln \frac{1+0.2 \delta}{1-0.2 \delta} \leq 0.5 \delta, \quad \text { and, analogously, } \\
& \ln \frac{\mu_{x \rightarrow a}^{[\omega+1]}(-1)}{\mu_{x \rightarrow a}^{[\omega+1]}(1)} \geq-0.5 \delta .
\end{aligned}
$$

Furthermore, since $x \notin T[\omega+1]$ Corollary 3.10 yields

$$
\left|\ln \frac{\mu_{a \rightarrow x}^{[\omega]}(-1)}{\mu_{a \rightarrow x}^{[\omega]}(1)}\right| \leq 2 \exp \left(-k_{1} / 2\right) \leq \delta^{2} .
$$

Hence,

$$
\left|\ln \frac{P(-1)}{P(1)}\right| \leq\left|\ln \frac{\mu_{x \rightarrow a}^{[\omega+1]}(-1)}{\mu_{x \rightarrow a}^{[\omega+1]}(1)}\right|+\left|\ln \frac{\mu_{a \rightarrow x}^{[\omega]}(-1)}{\mu_{a \rightarrow x}^{[\omega]}(1)}\right| \leq 0.5 \delta+\delta^{2} \leq 0.51 \delta
$$


Therefore, letting $z=\ln \frac{P(-1)}{P(1)}$, we obtain

$$
\left|\frac{1}{2}-\mu_{x}^{[\omega]}\left(\Phi_{t-1}\right)\right|=\left|\frac{1}{2}-\frac{P(1)}{P(-1)+P(1)}\right|=\left|\frac{1}{2}-\frac{1}{1+\exp (z)}\right| \leq\left|\frac{1-\exp (z)}{2}\right|<\delta,
$$

as desired.

\section{PROOF OF PROPOSITION 3.3}

The aim in this section is to prove Proposition 3.3 (i.e., to show that the random formula $\Phi$ is $\left(\delta_{t}, t\right)$ quasi-random with a sufficiently high probability). We proceed as follows: In Section 4.1, we introduce two alternative models of random $k$-SAT formulas to facilitate the proofs of the various quasi-randomness properties. Fact 4.1 establishes the formal relationship between $\boldsymbol{\Phi}$ and the two alternative models. Subsequently, in Sections 4.2 4.5, we prove that $\mathbf{Q 1 - Q 4}$ hold with a sufficient probability. We do not need to worry about Q0 because, in Proposition 3.3, we condition on this property. Sections 4.2-4.5 can be read independently of each other (i.e., we are not going to use partial results from one of these subsections in another). Ultimately, Proposition 3.3 will be immediate from Lemmas 4.7, 4.9, 4.17, and 4.20.

Throughout this section, we continue to use the notation from Sections 2 and 3, and we keep the assumptions of Proposition 3.3. In particular, we recall from Equation (14) that $\delta_{s}=\exp (-c(1-s / n) k)$ and that $\hat{t}=\left(1-\frac{\ln \rho}{c^{2} k}\right) n$. Suppose that $1 \leq t \leq \hat{t}$. Then, $\theta=1-t / n$ satisfies $\theta k \geq \ln (\rho) / c^{2}$. We continue to assume that $\rho=k r / 2^{k} \geq \rho_{0}$ for some large enough number $\rho_{0}$; in particular, we assume that $\rho_{0} \geq \exp (1 / c)$. We let

$$
\delta=\delta_{t}=\exp (-\operatorname{ck} \theta)
$$

for brevity. Then, Lemma 2.2 yields

$$
\delta \theta n>10^{15} \Delta_{t} .
$$

Finally, we tacitly assume that $n$ is sufficiently large.

\subsection{Models of Random Formulas}

To prove Proposition 3.3, two slightly different models of random $k$-CNFs come in handy. In the first "binomial" model $\boldsymbol{\Phi}_{\text {bin }}$, we obtain a $k$-CNF by including each of the $(2 n)^{k}$ possible clauses over $V=\left\{x_{1}, \ldots, x_{n}\right\}$ with probability $p=m /(2 n)^{k}$ independently, where each clause is an ordered $k$-tuple of not necessarily distinct literals. Thus, $\boldsymbol{\Phi}_{\text {bin }}$ is a random set of clauses, and $\mathrm{E}\left|\boldsymbol{\Phi}_{\text {bin }}\right|=m$.

In the second model, we choose a sequence $\boldsymbol{\Phi}_{\text {seq }}$ of $m$ independent $k$-clauses $\boldsymbol{\Phi}_{\text {seq }}(1), \ldots, \boldsymbol{\Phi}_{\text {seq }}(m)$ (with replacement), each of which consists of $k$ independently chosen literals. Thus, the probability of each individual sequence is $(2 n)^{-k m}$. The sequence $\boldsymbol{\Phi}_{\text {seq }}^{\prime}$ corresponds to the $k$-CNF $\left\{\boldsymbol{\Phi}_{\text {seq }}(1), \ldots, \boldsymbol{\Phi}_{\text {seq }}(m)\right\}$ with at most $m$ clauses. The following well-known fact relates $\boldsymbol{\Phi}$ to $\boldsymbol{\Phi}_{\text {bin }}, \boldsymbol{\Phi}_{\text {seq }}$ :

FACT 4.1. For any event $\mathcal{E}$ we have

$$
\begin{aligned}
& \mathrm{P}[\boldsymbol{\Phi} \in \mathcal{E}] \leq O(\sqrt{m}) \cdot \mathrm{P}\left[\boldsymbol{\Phi}_{\text {bin }} \in \mathcal{E}\right], \\
& \mathrm{P}[\boldsymbol{\Phi} \in \mathcal{E}] \leq O(1) \cdot \mathrm{P}\left[\boldsymbol{\Phi}_{\text {seq }} \in \mathcal{E}\right] .
\end{aligned}
$$

Due to Fact 4.1 and Equation (84), it suffices to prove that the statements Q1-Q4 hold for either of $\boldsymbol{\Phi}, \boldsymbol{\Phi}_{\text {bin }}, \boldsymbol{\Phi}_{\text {seq }}$ with probability at least $1-\exp \left(-10^{-13} \delta \theta n\right)$. We illustrate the use of Fact 4.1 by establishing bounds on the number of very short and very long clauses that will be used several times in the following.

LEMMA 4.2. With probability at least $1-\exp \left(-10^{-6} \delta \theta n\right)$ in $\Phi^{t}$ there are no more than $\theta n \cdot 10^{-5} \frac{\delta}{\theta k}$ clauses of length less than $0.1 \theta k$. 
Proof. We are going to work with $\boldsymbol{\Phi}_{\text {bin }}$. Let $L_{j}$ be the number of clauses of length $j$ in $\boldsymbol{\Phi}_{\text {bin }}^{t}$. Then, for any $j \in[k]$ we have

$$
\lambda_{j}=\mathrm{E}\left[L_{j}\right]=m \cdot 2^{j-k}\left(\begin{array}{c}
k \\
j
\end{array}\right) \theta^{j}(1-\theta)^{k-j}=\frac{2^{j} \rho \theta n}{j}\left(\begin{array}{c}
k-1 \\
j-1
\end{array}\right) \theta^{j-1}(1-\theta)^{k-j} .
$$

Indeed, a clause has length $j$ in $\boldsymbol{\Phi}_{\text {bin }}^{t}$ if and only if it contains $j$ variables from the set $V_{t}$ of size $\theta n$ and $k-j$ variables from $V \backslash V_{t}$ and none of the $k-j$ variables from $V \backslash V_{t}$ occurs positively. The total number of possible clauses with these properties is $2^{j}\left(\begin{array}{c}k \\ j\end{array}\right)(\theta n)^{j}((1-\theta) n)^{k-j} \rho$, and each of them is present in $\boldsymbol{\Phi}_{\text {bin }}^{t}$ with probability $p=m /(2 n)^{k}$ independently.

Let's start by bounding the total number $L_{*}=\sum_{j<\theta k / 10} L_{j}$ of "short" clauses. Its expectation is bounded by

$$
\begin{array}{rlrl}
\mathrm{E}\left[L_{*}\right] & =\sum_{j<\theta k / 10} \lambda_{j} \leq 2^{0.1 \theta k} \rho \theta n \cdot \mathrm{P}[\operatorname{Bin}(k-1, \theta)<\theta k / 10] \\
& \leq 2^{0.1 \theta k} \rho \theta n \cdot \exp (-\theta k / 3) & & {[\text { by Lemma } 1.8(\text { Chernoff })]} \\
& \leq \theta \exp (-\theta k / 4) n & & {\left[\text { as } \theta k \geq \ln (\rho) / c^{2}\right] .}
\end{array}
$$

Furthermore, $L_{*}$ is binomially distributed because clauses appear independently in $\boldsymbol{\Phi}_{\text {bin. }}$. Hence, again by Lemma 1.8, we have

$$
\begin{aligned}
\mathrm{P}\left[L_{*}>\theta n \cdot 10^{-5} \delta /(\theta k)\right] & \leq \exp \left[-\frac{10^{-5} \delta}{\theta k} \cdot \ln \left(\frac{10^{-5} \delta /(\theta k)}{\exp (1-\theta k / 4)}\right) \cdot \theta n\right] \\
& \leq \exp \left(-\frac{\delta}{5 \cdot 10^{5} \theta k} \cdot \theta k \cdot \theta n\right) \leq \exp \left(-2 \cdot 10^{-6} \delta \theta n\right) .
\end{aligned}
$$

Hence, the assertion follows from Equation (85) and Fact 4.1.

Corollary 4.3. With probability at least $1-\exp \left(-10^{-6} \delta \theta n\right)$, in $\boldsymbol{\Phi}^{t}$ no more than $10^{-6} \delta \theta n$ variables appear in clauses of length less than $0.1 \theta k$.

The following lemma deals with long clauses.

Lemma 4.4. With probability at least $1-\exp \left(-10^{-11} \delta \theta n\right)$, we have

$$
\sum_{b \in \Phi^{t}:|N(b)|>10 k \theta}|N(b)| \leq 10^{-6} \delta \theta n .
$$

Proof. For a given $\mu>0$, let $\mathcal{L}_{\mu}$ be the event that $\boldsymbol{\Phi}_{\text {seq }}^{t}$ has $\mu$ clauses so that the sum of the lengths of these clauses is at least $\lambda=10 \theta k \mu$. Then,

$$
\mathrm{P}\left[\mathcal{L}_{\mu}\right] \leq\left(\begin{array}{c}
m \\
\mu
\end{array}\right)\left(\begin{array}{c}
k \mu \\
\lambda
\end{array}\right) \theta^{\lambda}\left(\frac{1}{2}+\theta\right)^{k \mu-\lambda} .
$$

Indeed, there are $\left(\begin{array}{l}m \\ \mu\end{array}\right)$ ways to choose $\mu$ places for these $\mu$ clauses in $\boldsymbol{\Phi}_{\text {seq }}$. Once these have been specified, there are $k \mu$ literals that constitute the $\mu$ clauses, and we choose $\lambda$ whose underlying variables are supposed to be in $V_{t}$; the probability that this is indeed the case for all of these $\lambda$ literals is $\theta^{\lambda}$. Moreover, in order for each of the clauses to remain in $\Phi_{\text {seq }}^{t}$, the remaining $k \mu-\lambda$ literals must either be negative or have underlying 
variables from $V_{t}$, leading to the $(\theta+1 / 2)^{k \mu-\lambda}$ factor. Thus,

$$
\begin{array}{rlrl}
\mathrm{P}\left[\mathcal{L}_{\mu}\right] & \leq\left(\begin{array}{c}
m \\
\mu
\end{array}\right)\left[\left(\frac{1}{2}+\theta\right)\left(\frac{\mathrm{e}}{5}\right)^{10 \theta}\right]^{k \mu} & & {[\text { as } \lambda=10 k \theta \mu]} \\
& \leq\left(\frac{\mathrm{e} n \rho}{k \mu}\right)^{\mu}\left[(1+2 \theta)\left(\frac{\mathrm{e}}{5}\right)^{10 \theta}\right]^{k \mu} & & {\left[\text { as } m=n \cdot 2^{k} \rho / k\right]} \\
& \leq\left[\frac{\mathrm{e} \rho \rho}{k \mu}\left(\frac{\mathrm{e}}{4}\right)^{10 \theta k}\right]^{\mu} & \\
& =\left[\left(\frac{10 \mathrm{e} \rho \theta n}{\lambda}\right)^{1 /(10 \theta k)}\left(\frac{\mathrm{e}}{4}\right)\right]^{\lambda} & & {[\text { as } \lambda=10 k \theta \mu] .}
\end{array}
$$

Hence, if $\lambda \geq 10^{-6} \delta \theta n$ we get

$$
\begin{aligned}
\mathrm{P}\left[\mathcal{L}_{\mu}\right] & \leq\left[\left(\frac{10^{7} \mathrm{e} \rho}{\delta}\right)^{1 /(10 \theta k)}\left(\frac{\mathrm{e}}{4}\right)\right]^{\lambda} \leq\left(\frac{\mathrm{e}}{3}\right)^{\lambda} \quad\left[\text { as } k \theta \geq \ln (\rho) / c^{2} \text { and } \delta=\exp (-c k \theta)\right] \\
& \leq \exp \left(-10^{-10} \delta \theta n\right) .
\end{aligned}
$$

Thus, we see that in $\boldsymbol{\Phi}_{\text {seq }}^{t}$ with probability at least $1-\exp \left(-10^{-10} \delta \theta n\right)$, we have

$$
\sum_{b:|N(b)|>10 k \theta}|N(b)| \leq 10^{-6} \delta \theta n .
$$

Hence, Fact 4.1 implies that Equation (86) holds in $\Phi^{t}$ with probability at least 1 $\exp \left(-10^{-11} \delta \theta n\right)$.

COROLlary 4.5. With probability at least $1-\exp \left(-10^{-11} \delta \theta n\right)$, no more than $10^{-6} \delta \theta n$ variables appear in clauses of length greater than $10 \theta k$.

Proof. The number of such variables is bounded by $\sum_{b:|N(b)|>10 \theta k}|N(b)|$. Therefore, the assertion follows from Lemma 4.4.

We end this section with the following simple observation:

Lemma 4.6. Let $x \in V_{t}$. The expected number of clauses of length $j$ in $\boldsymbol{\Phi}_{\text {bin }}^{t}$ where $x$ is the underlying variable of the lth literal is

$$
\mu_{j}=\rho \cdot \frac{2^{j}}{j}\left(\begin{array}{c}
k-1 \\
j-1
\end{array}\right) \theta^{j-1}(1-\theta)^{k-j+1} \leq 2^{j} \rho / j .
$$

Proof. There are $2^{j}\left(\begin{array}{c}k \\ j\end{array}\right)(\theta n)^{j-1}((1-\theta) n)^{k-j}$ possible clauses that have exactly $j$ literals whose underlying variable is in $V_{t}$ such that the underlying variable of the $l$ th such literal is $x$. Each such clause is present in $\boldsymbol{\Phi}_{\text {bin }}$ with probability $p=m /(2 n)^{k}=\frac{\rho}{k} n^{1-k}$ independently.

\subsection{Establishing Q1}

We recall that $\mathbf{Q 1}$ states the following:

No more than $10^{-5} \delta \theta$ n variables occur in clauses of length less than $\theta k / 10$ or greater than $10 \theta k$ in $\Phi^{t}$. Moreover, there are at most $10^{-4} \delta \theta n$ variables $x \in V_{t}$ such that

$$
(\theta k)^{3} \delta \cdot \sum_{b \in N(x)} 2^{-|N(b)|}>1 .
$$

The main result of this section is 
Lemma 4.7. For all $1 \leq t \leq \hat{t}$ we have

$$
\mathrm{P}\left[\boldsymbol{\Phi}^{t} \text { satisfies } \mathbf{Q 1} \mid \boldsymbol{\Phi} \text { is tame }\right] \geq 1-\exp \left[-10 \Delta_{t}-10^{-13} \delta \theta n\right] .
$$

Since Corollary 4.3 and Corollary 4.5 already provide bounds on the numbers of variables in very short or very long clauses, the following lemma is the main step toward proving Lemma 4.7.

LeMmA 4.8. With probability at least $1-\exp \left(-10^{-12} \delta \theta n\right)$, no more than $10^{-4} \delta \theta n$ variables $x \in V_{t}$ are such that $\delta(\theta k)^{3} \sum_{b \in N(x)} 2^{-|N(b)|}>1$.

Proof. For $x \in V_{t}$ let $X_{j}(x)$ be the number of clauses of length $j$ in $\boldsymbol{\Phi}_{\text {bin }}^{t}$ that contain $x$, and let $X_{j l}(x)$ be the number of such clauses where $x$ is the underlying variable of the $l$ th literal of that clause $(1 \leq l \leq j)$. Then $\mathrm{E}\left[X_{j l}(x)\right]=\mu_{j}$, with $\mu_{j}$ as in Equation (87). Since $1 / \delta=\exp (c k \theta)$ and $k \theta \geq \ln (\rho) / c^{2}$, we see that $2^{j} \delta^{-1}(\theta k)^{-5} / j>100 \mu_{j}$. Hence, Lemma 1.8 (the Chernoff bound) yields

$$
\mathrm{P}\left[X_{j l}(x)>10\left(\mu_{j}+2^{j} \delta^{-1}(\theta k)^{-5} / j\right)\right] \leq \zeta, \quad \text { with } \zeta=\exp \left(-10 /\left(\delta(\theta k)^{5}\right)\right) .
$$

Let $V_{j l}$ be the set of all variables $x \in V_{t}$ such that $X_{j l}(x)>10\left(\mu_{j}+2^{j} \delta^{-1}(\theta k)^{-5} / j\right)$. Since the random variables $\left(X_{j l}(x)\right)_{x \in V_{t}}$ are mutually independent, Lemma 1.8 (the Chernoff bound) yields

$$
\mathrm{P}\left[\left|V_{j l}\right|>\frac{\delta}{(\theta k)^{9}} \cdot \theta n\right] \leq \exp \left[-\frac{\delta \theta n}{(\theta k)^{9}} \cdot \ln \left(\frac{\delta}{\mathrm{e}(\theta k)^{9} \zeta}\right)\right]
$$

Since $\zeta^{-1}=\exp \left(10 /\left(\delta(\theta k)^{5}\right)\right)=\exp \left[10 \exp (\operatorname{ck} \theta) /(\theta k)^{5}\right]$ and $k \theta \geq \ln (\rho) / c^{2} \gg 1$, we have

$$
\ln \left(\frac{\delta}{\mathrm{e}(\theta k)^{9} \zeta}\right) \geq-\ln (\zeta) / 2
$$

whence

$$
\mathrm{P}\left[\left|V_{j l}\right|>\frac{\delta}{(\theta k)^{9}} \cdot \theta n\right] \leq \exp \left[\frac{\delta \theta n}{2(\theta k)^{9}} \cdot \ln \zeta\right] \leq \exp \left[-\frac{\theta n}{(\theta k)^{15}}\right] \leq \exp (-\delta \theta n) .
$$

Furthermore, if $x \notin V_{j l}$ for all $1 \leq j \leq 10 \theta k$ and all $1 \leq l \leq j$, then

$$
\begin{aligned}
\sum_{b \in N(x):|N(b)| \leq 10 \theta k} 2^{-|N(b)|} & \leq 10 \sum_{j \leq 10 \theta k} 2^{-j}\left(j \mu_{j}+2^{j} \delta^{-1}(\theta k)^{-5}\right) \\
& \leq 100 \delta^{-1}(\theta k)^{-4}+10 \sum_{j \leq 10 \theta k} j 2^{-j} \mu_{j} \\
& \leq 100 \delta^{-1}(\theta k)^{-4}+10 \rho<\delta^{-1}(\theta k)^{-3},
\end{aligned}
$$

where we used that $\theta k \geq \ln (\rho) / c^{2}$, so that $1 / \delta \geq(\theta k)^{5} \rho$. Hence, the assertion follows from Equation (88), Fact 4.1, and the bound on the number of variables in clauses of length $>10 \theta k$ provided by Lemma 4.4 .

Proof of Lemma 4.7. The assertion is immediate from Corollary 4.3, Corollary 4.5, Lemma 4.8, and Equation (84). 


\subsection{Establishing Q2}

We recall that $\mathbf{Q} 2$ provides the following:

If $T \subset V_{t}$ has size $|T| \leq \delta \theta n$, then there are no more than $10^{-4} \delta \theta$ n variables $x$ such that either

$$
\begin{aligned}
& \sum_{b \in N_{1}(x, T)} 2^{-|N(b)|}>\rho(\theta k)^{5} \delta, \text { or } \sum_{b \in N_{>1}(x, T)} 2^{|N(b) \cap T \backslash\{x\}|-|N(b)|}>\frac{\delta}{\theta k}, \text { or } \\
& \left|\sum_{b \in N_{\leq 1}(x, T)} \frac{\operatorname{sign}(x, b)}{2^{|N(b)|}}\right|>\frac{\delta}{1000} .
\end{aligned}
$$

In this section we prove:

Lemma 4.9. For all $1 \leq t \leq \hat{t}$ we have

$$
\mathrm{P}\left[\boldsymbol{\Phi}^{t} \text { satisfies } \mathbf{Q 2} \mid \boldsymbol{\Phi} \text { is tame }\right] \geq 1-\exp \left[-10 \Delta_{t}-10^{-13} \delta \theta n\right] \text {. }
$$

Thus, let $T \subset V_{t}$ be a set of size $|T| \leq \delta \theta n$. For a variable $x$, we let $\mathcal{Q}(x, i, j, l, T)$ be the number of clauses $b$ of $\boldsymbol{\Phi}_{\text {bin }}^{t}$ such that the $i$ th literal is either $x$ or $\neg x,|N(b)|=j$, and $|N(b) \cap T \backslash\{x\}|=l$.

Lemma 4.10. Suppose that $l \geq 1, j-l>k_{1}$ and $0.1 \theta k \leq j \leq 10 \theta k$. Let

$$
\gamma_{j, l}=\left\{\begin{array}{cl}
10 j 2^{j} \delta \rho & \text { if } l=1 \\
102^{j-l} \delta^{1.9} & \text { if } l>1
\end{array}\right.
$$

Then, for any $i, x, T$, we have $\mathrm{P}\left[\mathcal{Q}(x, i, j, l, T)>\gamma_{j, l}\right] \leq \exp \left(-\exp \left(c^{2 / 3} \theta k\right)\right)$.

Proof. The random variable $\mathcal{Q}(x, i, j, l, T)$ has a binomial distribution because clauses appear independently in $\boldsymbol{\Phi}_{\text {bin }}$. With $\mu_{j}$ from Equation (87), we have for $l>1$

$$
\mathrm{E}[\mathcal{Q}(x, i, j, l, T)] \leq\left(\begin{array}{l}
j \\
l
\end{array}\right) \delta^{l} \mu_{j} \leq \rho\left(\begin{array}{l}
j \\
l
\end{array}\right) \delta^{l} 2^{j} \leq 2^{j-l} \delta^{1.9} ;
$$

in the last step, we used that $\delta^{0.05} \leq 1 / \rho$, which follows from our assumption that $\theta k \geq \ln (\rho) / c^{2}$, and that $2^{l}\left(\begin{array}{l}j \\ l\end{array}\right) \leq(2 j)^{l} \leq(20 k \theta)^{l} \leq \delta^{0.05 l}$. Hence, by Lemma 1.8 (the Chernoff bound) in the case $j-l>k_{1}=\sqrt{c} \theta k, l>1$ we get

$$
\mathrm{P}\left[\mathcal{Q}(x, i, j, l, T)>10 \cdot 2^{j-l} \delta^{1.9}\right] \leq \exp \left(-2^{j-l} \delta^{1.9}\right) \leq \exp \left(-2^{k_{1}} \delta^{1.9}\right) \leq \exp \left(-\exp \left(c^{2 / 3} \theta k\right)\right),
$$
as $\delta=\exp (-\operatorname{ck} \theta)$.

By a similar token, in the case $l=1$, we have $\mathrm{E}[\mathcal{Q}(x, i, j, l, T)] \leq j \delta^{l} \mu_{j} \leq \rho \delta 2^{j}$. Hence, once more by the Chernoff bound

$$
\mathrm{P}\left[\mathcal{Q}(x, i, j, l, T)>10 \cdot 2^{j} \delta \rho\right] \leq \exp \left(-2^{j} \delta \rho\right) \leq \exp \left(-2^{k_{1}} \delta\right) \leq \exp \left(-\exp \left(c^{2 / 3} \theta k\right)\right),
$$

as claimed.

Let $\mathcal{Z}(i, j, l, T)$ be the number of variables $x \in V_{t}$ for which $\mathcal{Q}(x, i, j, l, T)>\gamma_{j, l}$.

Lemma 4.11. Suppose that $l \geq 1, j-l>k_{1}$ and $0.1 \theta k \leq j \leq 10 \theta k$. Then, for any $i, T$ we have

$$
\mathrm{P}\left[\mathcal{Z}(i, j, l, T)>\delta \theta n /(\theta k)^{4}\right] \leq \exp \left[-\frac{\delta \theta n}{2(\theta k)^{4}} \cdot \exp \left(c^{2 / 3} \theta k\right)\right] .
$$

Proof. Whether a variable $x \in V_{t}$ contributes to $\mathcal{Z}(i, j, l, T)$ depends only on those clauses of $\boldsymbol{\Phi}_{\text {bin }}^{t}$ whose $i$ th literal reads either $x$ or $\neg x$. Since these sets of clauses 
are disjoint for distinct variables, and because clauses appear independently in $\boldsymbol{\Phi}_{\text {bin }}$, $\mathcal{Z}(i, j, l, T)$ is a binomial random variable. By Lemma 4.10,

$$
\mathrm{E}[\mathcal{Z}(i, j, l, T)] \leq \theta n \exp \left(-\exp \left(c^{2 / 3} \theta k\right)\right) .
$$

Hence, Lemma 1.8 (the Chernoff bound) yields

$$
\begin{aligned}
\mathrm{P}\left[\mathcal{Z}(i, j, l, T)>\delta \theta n /(\theta k)^{4}\right] & \leq \exp \left[-\frac{\delta \theta n}{(\theta k)^{4}} \ln \left(\frac{\delta}{(\theta k)^{4} \exp \left(1-\exp \left(c^{2 / 3} \theta k\right)\right)}\right)\right] \\
& \leq \exp \left[-\frac{\delta \theta n}{2(\theta k)^{4}} \cdot \exp \left(c^{2 / 3} \theta k\right)\right],
\end{aligned}
$$

as desired.

Corollary 4.12. With probability $1-\exp (-\delta \theta n)$, the random formula $\boldsymbol{\Phi}_{\text {bin }}$ has the following property:

For all $i, j, l, T$ such that $l \geq 1, j-l>k_{1}, 0.1 \theta k \leq j \leq 10 \theta k$, and $|T| \leq \delta \theta n$ we have $\mathcal{Z}(i, j, l, T) \leq \delta \theta n /(\theta k)^{4}$.

Proof. We apply the union bound. There are at most $n\left(\begin{array}{c}n \\ \delta n\end{array}\right)$ ways to choose the set $T$, and no more than $n$ ways to choose $i, j, l$. Hence, by Lemma 4.11 , the probability that there exist $i, j, l, T$ such that $\mathcal{Z}(i, j, l, T)>\theta n \exp \left(-\exp \left(c^{2 / 3} \theta k\right)\right)$ is bounded by

$$
\begin{aligned}
n^{2}\left(\begin{array}{c}
n \\
\delta \theta n
\end{array}\right) \exp \left[-\frac{\delta \theta n}{(\theta k)^{4}}\right. & \left.\cdot \exp \left(c^{2 / 3} \theta k\right)\right] \\
& \leq \exp \left[o(n)+\delta \theta n(1-\ln (\delta \theta))-\frac{\delta \theta n}{(\theta k)^{4}} \cdot \exp \left(c^{2 / 3} \theta k\right)\right] \\
& \leq \exp \left[\delta \theta n\left[o(1)-2 \ln \delta-\exp \left(c^{3 / 4} \theta k\right)\right] \leq \exp [-\delta \theta n],\right.
\end{aligned}
$$

as claimed.

Corollary 4.13. With probability $1-\exp \left(-10^{-12} \delta \theta n\right)$, the random formula $\Phi^{t}$ has the following property:

If $T \subset V_{t}$ has size $|T| \leq \delta \theta n$, then for all but $\delta \theta n / 30000$ variables $x$ we have

$$
\sum_{b \in N_{>1}(x, T)} 2^{|N(b) \cap T \backslash\{x\}|-|N(b)|}<\frac{\delta}{\theta k} \text { and } \sum_{b \in N_{1}(x, T)} 2^{-|N(b)|}<\rho(\theta k)^{5} \delta .
$$

Proof. Given $T \subset V_{t}$ of size $|T| \leq \delta \theta n$, let $\mathcal{V}_{T}$ be the set of all variables $x$ with the following two properties.

i. For all $b \in N(x)$, we have $0.1 \theta k \leq|N(b)| \leq 10 \theta k$.

ii. For all $1 \leq i \leq j, 1 \leq l \leq j-k_{1}$, and $0.1 \theta \bar{k} \leq j \leq 10 \theta k$, we have $\mathcal{Q}(x, i, j, l, T) \leq \gamma_{j, l}$.

Then, for all $x \in \mathcal{V}_{T}$, we have

$$
\begin{array}{rlrl}
\sum_{b \in N_{>1}(x, T)} 2^{|N(b) \cap T \backslash\{x\}|-|N(b)|} & =\sum_{0.1 k \theta \leq j \leq 10 k \theta} \sum_{i=1}^{j} \sum_{l=2}^{j-k_{1}} \mathcal{Q}(x, i, j, l, T) 2^{l-j} \quad \text { [due to (i)] } \\
& \leq 10 k \theta \sum_{0.1 k \theta \leq j \leq 10 k \theta} \sum_{l=2}^{j-k_{1}} \gamma_{j, l} 2^{l-j} & \text { [due to (ii)] } \\
& \leq 1000(k \theta)^{2} \delta^{1.9}<\delta /(k \theta) & \text { [as } \delta=\exp (-c k \theta)] .
\end{array}
$$


Similarly,

$$
\begin{aligned}
\sum_{b \in N_{1}(x, T)} 2^{-|N(b)|} & \leq \sum_{0.1 k \theta \leq j \leq 10 k \theta} \sum_{i=1}^{j} \mathcal{Q}(x, i, j, 1, T) 2^{-j} & \text { [due to (i)] } \\
& \leq 10 k \theta \sum_{0.1 k \theta \leq j \leq 10 k \theta} 2^{-j} \gamma_{j, 1} & \text { [due to (ii)] } \\
& \leq 1000(k \theta)^{3} \delta \rho<\rho(k \theta)^{5} \delta & {\left[\text { as } k \theta \geq \ln (\rho) / c^{2} \gg 1\right] . }
\end{aligned}
$$

Thus, to complete the proof, we need to show that with sufficiently high probability $\mathcal{V}_{T}$ is sufficiently big for all $T$. By Lemmas 4.2 and 4.4 with probability $1-2 \exp \left(-10^{-11} \delta \theta n\right)$, the number of variables $x$ that fail to satisfy (i) is less than $2 \cdot 10^{-6} \delta \theta n$. Furthermore, by Corollary 4.12 and Fact 4.1 , with probability $\geq 1-\exp (-\delta \theta n / 2)$, the random formula $\Phi^{t}$ satisfies Equation (89). In this case, for all $\bar{T}$, the number of variables that fail to satisfy (ii) is bounded by $\delta \theta n /(k \theta)^{4}<10^{-5} \delta \theta n$. Thus, with probability $\geq 1-\exp \left(-10^{-12} \delta \theta n\right)$, we have $\left|\mathcal{V}_{T}\right|>\theta n(1-\delta / 30000)$ for all $T$, as desired.

For a set $T \subset V_{t}$ and numbers $i \leq j$, we let $\mathcal{N}_{+}(x, i, j, T)$ be the number of clauses $b \in N(x)$ in $\boldsymbol{\Phi}_{\text {bin }}^{t}$ such that $|N(b)|=j$, the $i$ th literal of $b$ is $x$, and $|N(b) \cap T \backslash x| \leq 1$. Similarly, we let $\mathcal{N}_{-}(x, i, j, T)$ be the number of $b \in N(x)$ such that $|N(b)|=j$, the $i$ th literal of $b$ is $\neg x$, and $|N(b) \cap T \backslash x| \leq 1$. Let $\mathcal{B}(i, j, T)$ be the set of variables $x \in V_{t}$ such that

$$
\left|\mathcal{N}_{+}(x, i, j, T)-\mathcal{N}_{-}(x, i, j, T)\right|>2^{j} \delta(\theta k)^{-3} .
$$

Lemma 4.14. Let $T \subset V_{t}$ be a set of size $|T| \leq \delta \theta n$. Let $i, j$ be such that $i \leq j$ and $0.1 \theta k \leq j \leq 10 \theta k$. Then, in $\boldsymbol{\Phi}_{\text {bin }}^{t}$ we have

$$
\mathrm{P}\left[\mathcal{B}(i, j, T)>\delta \theta n /(\theta k)^{3}\right] \leq \exp [-\delta \theta n \exp (\theta k / 22)] .
$$

PRoof. Let $x \in V_{t}$. In the random formula $\boldsymbol{\Phi}_{\text {bin }}^{t}$, we have

$$
\mathrm{E}\left[\mathcal{N}_{+}(x, i, j, T)+\mathcal{N}_{-}(x, i, j, T)\right] \leq \mu_{j} \leq 2^{j} \rho / j \quad \text { (with } \mu_{j} \text { as in Equation (87)). }
$$

Furthermore, $\mathcal{N}_{+}(x, i, j, T), \mathcal{N}_{-}(x, i, j, T)$ are binomially distributed with identical means because in $\boldsymbol{\Phi}_{\text {bin }}$ each literal is positive/negative with probability $\frac{1}{2}$. Hence, for $j \geq 0.1 \theta k$ Lemma 1.8 (the Chernoff bound) yields

$$
\begin{aligned}
\mathrm{P}\left[\mid \mathcal{N}_{+}(x, i, j, T)-\mathcal{N}_{-}(x,\right. & \left.i, j, T) \mid>2^{j} \delta(\theta k)^{-3}\right] \leq \exp \left[-\frac{\left(2^{j} \delta(\theta k)^{-3}\right)^{2}}{3\left(2^{j} \delta(\theta k)^{-3}+2^{j} \rho\right)}\right] \\
& \leq \exp \left[-\frac{2^{j} \delta^{2}}{4(\theta k)^{6} \rho}\right] \\
& \leq \exp (-\exp (\theta k / 20)) \quad[\operatorname{as} \delta=\exp (-c k \theta), j \geq 0.1 \theta k] .
\end{aligned}
$$

For different variables $x \in V_{t}$ the random variables $\mathcal{N}_{+}(x, i, j, T)-\mathcal{N}_{-}(x, i, j, T)$ are independent (because we fix the position $i$ where $x$ occurs). Hence, $\mathcal{B}(i, j, T)$ is a binomial random variable, and Equation (90) yields

$$
\mathrm{E}[\mathcal{B}(i, j, T)] \leq \theta n \exp (-\exp (\theta k / 20))
$$


Consequently, Lemma 1.8 (the Chernoff bound) gives

$$
\begin{aligned}
\mathrm{P}\left[\mathcal{B}(i, j, T)>\theta \delta n /(\theta k)^{3}\right] & \leq \exp \left[-\frac{\delta \theta n}{(\theta k)^{3}} \ln \left(\frac{\delta \theta n /(\theta k)^{3}}{\exp (1-\exp (\theta k / 20)) \theta n}\right)\right] \\
& \leq \exp \left[-\frac{\delta \theta n}{(\theta k)^{3}} \cdot \exp (\theta k / 21)\right] \leq \exp [-\theta \delta n \exp (\theta k / 22)]
\end{aligned}
$$

provided that $\rho \geq \rho_{0}$ is sufficiently large.

Corollary 4.15. With probability $\geq 1-\exp (-\delta \theta n)$, the random formula $\boldsymbol{\Phi}_{\text {bin }}^{t}$ has the following property.

For all $T \subset V_{t}$ of size $|T| \leq \delta \theta n$ and all $i, j$ such that $i \leq j, 0.1 \theta k \leq j \leq 10 \theta k$ we have $\mathcal{B}(i, j, T) \leq \delta \theta n /(\theta k)^{3}$.

Proof. Let $i, j$ be such that $i \leq j, 0.1 \theta k \leq j \leq 10 \theta k$. By Lemma 4.14 and the union bound, the probability that there is a set $T$ such that $\mathcal{B}(i, j, T)>\delta \theta n /(\theta k)^{3}$ is bounded by

$$
\begin{aligned}
n\left(\begin{array}{c}
\theta n \\
\delta \theta n
\end{array}\right) \exp [-\delta \theta n \exp (\theta k / 22)] & \leq \exp [o(n)+\delta \theta n(1-\ln (\theta \delta)-\exp (\theta k / 22))] \\
& \leq \exp [-2 \delta \theta n] \quad[\text { as } \delta=\exp (-\operatorname{ck} \theta)] .
\end{aligned}
$$

Since there are no more than $(10 k \theta)^{2}$ ways to choose $i, j$, the assertion follows.

Corollary 4.16. With probability $\geq 1-\exp \left(-10^{-12} \delta \theta n\right)$, the random formula $\Phi^{t}$ has the following property.

If $T \subset V_{t}$ has size $|T| \leq \delta \theta n$, then there are no more than $\delta \theta n / 30000$ variables $x \in V_{t}$ such that

$$
\left|\sum_{b \in N_{\leq 1}(x, T)} \frac{\operatorname{sign}(x, b)}{2^{|N(b)|}}\right|>\frac{\delta}{1000} .
$$

Proof. Given $T \subset V_{t}$, let $\mathcal{W}_{T}$ be the set of all $x \in V_{t}$ with the following two properties:

i. For all $b \in N(x)$, we have $0.1 \theta k \leq|N(b)| \leq 10 \theta k$.

ii. For all $1 \leq i \leq j, 0.1 \theta k \leq j \leq 10 \theta k$, we have $\mathcal{B}(i, j, T) \leq \delta \theta n /(\theta k)^{3}$.

Then, for all $x \in \mathcal{W}_{T}$ we have

$$
\begin{aligned}
\left|\sum_{b \in N_{\leq 1}(x)} \frac{\operatorname{sign}(x, b)}{2^{|N(b)|}}\right| & =\left|\sum_{0.1 \theta} \sum_{k \leq j \leq 10 \theta k} \sum_{i=1}^{j} 2^{-j}\left(\mathcal{N}_{+}(x, i, j, T)-\mathcal{N}_{-}(x, i, j, T)\right)\right| \\
& \leq \sum_{0.1 \theta k \leq j \leq 10 \theta k} \sum_{i=1}^{j} 2^{-j}\left|\mathcal{N}_{+}(x, i, j, T)-\mathcal{N}_{-}(x, i, j, T)\right| \\
& \leq 10 \theta k \sum_{0.1 \theta k \leq j \leq 10 \theta k} 2^{-j} \cdot 2^{j} \delta(\theta k)^{-3} \quad \text { [due to (ii)] } \\
& \leq 100 \delta /(\theta k)<10^{-3} \delta .
\end{aligned}
$$

Furthermore, by Lemmas 4.2 and 4.4 with probability $\geq 1-2 \exp \left(-10^{-11} \delta \theta n\right)$, the number of variables $x$ that fail to satisfy i. is less than $2 \cdot 10^{-6} \delta \theta n$. In addition, by Corollary 4.15 and Fact 4.1 with probability $\geq 1-\exp (-\delta \theta n / 2)$, the number of variables 
$x$ that fail to satisfy (ii) in $\Phi^{t}$ is bounded by $10^{-5} \delta \theta n$. Thus, with probability $\geq 1-$ $\exp \left(-10^{-12} \delta \theta n\right)$, we have $\left|\mathcal{W}_{T}\right| \geq(1-\delta / 30000) \theta n$.

Proof of Lemma 4.9. The assertion is immediate from Corollary 4.13, Corollary 4.16, and Equation (84).

\subsection{Establishing Q3}

We recall condition Q3:

For any $0.01 \leq z \leq 1$ and any set $T \subset V_{t}$ of size $|T| \leq 100 \delta \theta n$, we have

$$
\sum_{b:|N(b) \cap T| \geq z|N(b)|}|N(b)| \leq \frac{1.01}{z}|T|+10^{-4} \delta \theta n .
$$

The purpose of this section is to establish the following:

LeMma 4.17. For all $1 \leq t \leq \hat{t}$ we have

$$
\mathrm{P}\left[\boldsymbol{\Phi}^{t} \text { satisfies } \mathbf{Q 3} \mid \boldsymbol{\Phi} \text { is tame }\right] \geq 1-\exp \left[-10 \Delta_{t}-10^{-13} \delta \theta n\right] \text {. }
$$

We carry the proof out in the model $\boldsymbol{\Phi}_{\text {seq }}$. Let $0.01 \leq z \leq 1$ and let $T$ be a set of size $|T|=q \theta n$ with $0.01 \delta \leq q \leq 100 \delta$.

LemMa 4.18. Let $S, Z>0$ be integers and let $\mathcal{E}_{z}(T, S, Z)$ be the event that $\Phi_{\text {seq }}^{t}$ contains a set $\mathcal{Z}$ of $Z$ clauses with the following properties:

i. $S=\sum_{b \in \mathcal{Z}}|N(b)|>1.009|T| / z$,

ii. For all $b \in \mathcal{Z}$, we have $0.1 \theta k \leq|N(b)| \leq 10 \theta k$.

iii. All $b \in \mathcal{Z}$ satisfy $|N(b) \cap T| \geq z|N(b)|$.

Then, $\mathrm{P}\left[\mathcal{E}_{z}(T, S, Z)\right] \leq q^{0.99999 z S}$.

Proof. We claim that in $\boldsymbol{\Phi}_{\text {seq }}^{t}$,

$$
\mathrm{P}\left[\mathcal{E}_{z}(T, S, Z)\right] \leq\left(\begin{array}{c}
m \\
Z
\end{array}\right)\left(\begin{array}{c}
k Z \\
S
\end{array}\right)\left(\begin{array}{c}
S \\
z S
\end{array}\right) 2^{S-k Z} \theta^{S}(1-\theta)^{k Z-S} q^{z S} .
$$

Indeed, $\boldsymbol{\Phi}_{\text {seq }}^{t}$ is based on the random sequence $\boldsymbol{\Phi}_{\text {seq }}$ of $m$ independent clauses. Out of these $m$ clauses, we choose a subset $\mathcal{Z}$ of size $Z$, inducing a $\left(\begin{array}{c}m \\ Z\end{array}\right)$ factor. Then, out of the $k Z$ literal occurrences of the clauses in $\mathcal{Z}$ we choose $S$ (leading to the $\left(\begin{array}{c}k Z \\ S\end{array}\right)$ factor) whose underlying variables lie in $V_{t}$, which occurs with probability $\theta=\left|V_{t}\right| / n$ independently for each literal (inducing a $\theta^{S}$ factor). Furthermore, all $k Z-S$ literals whose variables are in $V \backslash V_{t}$ must be negative because otherwise the corresponding clauses would have been eliminated from $\boldsymbol{\Phi}_{\text {seq }}^{t}$; this explains the $2^{S-k Z}(1-\theta)^{k Z-S}$ factor. Finally, out of the $S$ literal occurrences in $V_{t}$ a total of at least $z S$ has an underlying variable from $T$ (a factor of $\left(\begin{array}{c}S \\ z S\end{array}\right)$ ), which occurs with probability $q=|T| /(\theta n)$ independently (hence the $q^{z S}$ factor).

Hence, we obtain

$$
\begin{aligned}
\mathrm{P}\left[\mathcal{E}_{z}(T, S, Z)\right] & \leq\left(\begin{array}{c}
m \\
Z
\end{array}\right) 2^{-k Z}\left[2^{1 / z} \cdot \frac{\mathrm{e}}{z} \cdot q\right]^{z S} \cdot\left(\begin{array}{c}
k Z \\
S
\end{array}\right) \theta^{S}(1-\theta)^{k Z-S} \\
& \leq\left(\begin{array}{c}
m \\
Z
\end{array}\right) 2^{-k Z}\left[2^{1 / z} \cdot \frac{\mathrm{e}}{z} \cdot q\right]^{z S} \leq\left(\begin{array}{c}
m \\
Z
\end{array}\right) 2^{-k Z}(C q)^{z S}
\end{aligned}
$$

for a certain absolute constant $C>0$ because $z \geq 0.01$. Since all clause lengths are required to be between $0.1 \theta k$ and $10 \theta k$, we obtain $0.1 S /(\theta k) \leq Z \leq 10 S /(\theta k)$. Therefore, 


$$
\begin{aligned}
\left(\begin{array}{c}
m \\
Z
\end{array}\right) 2^{-k Z} & \leq\left(\frac{\mathrm{e} m}{2^{k} Z}\right)^{Z} \leq\left(\frac{\mathrm{e} \rho n}{k Z}\right)^{Z} \quad\left[\text { as } m=2^{k} \rho n / k\right] \\
& \leq\left(\frac{10 \mathrm{e} \rho \theta n}{S}\right)^{Z} \\
& \leq\left(\frac{10 \mathrm{e} \rho}{1.009 q}\right)^{Z} \quad[\text { as } S \geq 1.009 q \theta n / z \geq 1.009 q \theta n \text { by (i)]. }
\end{aligned}
$$

Since $q \leq 100 \delta=100 \exp (-c \theta k)$ and $\theta k \geq \ln (\rho) / c^{2}$, we have $1 / q \geq 100 \rho$ for $\rho \geq \rho_{0}$ sufficiently large. Hence, Equation (94) yields

$$
\left(\begin{array}{c}
m \\
Z
\end{array}\right) 2^{-k Z} \leq q^{-2 Z} \leq q^{-20 S /(\theta k)}
$$

Plugging Equation (95) into Equation (93), we obtain for $\theta k \geq \rho_{0}$ large enough and $S \geq 1.009|T| / z$

$$
\mathrm{P}\left[\mathcal{E}_{z}(T, S, Z)\right] \leq q^{-20 S /(\theta k)} \cdot(C q)^{z S} \leq q^{0.99999 z S}
$$

as claimed.

Corollary 4.19. Let $\mathcal{E}$ be the event that there exist a number $z \in[0.01,1]$, a set $T \subset V_{t}$ of size $|T| \leq 100 \theta \delta$ n and $S \geq \frac{1.01}{z}|T|+10^{-6} \delta \theta n, Z>0$ such that $\mathcal{E}_{z}(T, S, Z)$ occurs. Then $\mathcal{E}$ occurs in $\boldsymbol{\Phi}^{t}$ with probability $\leq \exp \left(-10^{-7} \delta \theta n\right)$.

Proof. Let $z \in[0.01,1]$, let $0<q \leq 100 \delta$, and let $S, Z>0$ be integers such that $S \geq \frac{1.01}{z} q \theta n+10^{-6} \delta \theta n$. Let $\mathcal{E}_{z}(q, S, Z)$ denote the event that there is a set $T \subset V_{t}$ of size $|T|=q \theta n$ such that $\mathcal{E}_{z}(T, S, Z)$ occurs. Then, by Lemma 4.18 and the union bound, in $\boldsymbol{\Phi}_{\text {seq }}^{t}$ we have

$$
\begin{aligned}
& \left.\mathrm{P}[\mathcal{E}(q, S, Z)] \leq\left(\begin{array}{c}
\theta n \\
q \theta n
\end{array}\right) q^{0.99999 z S} \leq \exp [q \theta n(1-\ln q+1.008 \ln q))+0.9 \cdot 10^{-6} \delta \theta n \ln q\right] \\
& \left.\leq \exp \left(-0.9 \cdot 10^{-6} \delta \theta n\right) \quad \text { [as } q \leq 100 \delta<1 / \mathrm{e}\right]
\end{aligned}
$$

Since there are only $O\left(n^{4}\right)$ possible choices of $S, Z, z$ and $q$, Equation (96) and Fact 4.1 imply the assertion.

Proof of Lemma 4.17. Due to Equation (84), it suffices to show that with probability at least $1-\exp \left(-10^{-12} \delta \theta n\right), \boldsymbol{\Phi}^{t}$ has the following property:

Let $0.01 \leq z \leq 1$ and let $T \subset V_{t}$ have size $0.01 \delta \theta n \leq|T| \leq 100 \delta \theta n$. Then

$$
\sum_{b:|N(b) \cap T| \geq z|N(b)|}|N(b)| \leq \frac{1.01}{z}|T|+2 \cdot 10^{-5} \delta \theta n .
$$

To prove that this is true, we observe that Lemmas 4.2 and 4.4 and Corollary 4.19 imply that with probability at least $1-3 \exp \left(-10^{-11} \delta \theta n\right), \boldsymbol{\Phi}^{t}$ has the following properties:

i. $\mathcal{E}$ does not occur.

ii. $\sum_{b:|N(b)| \notin[0.1 \theta k, 10 \theta k]}|N(b)| \leq 10^{-5} \delta \theta n$.

Assume that (i) and (ii) hold and let $T \subset V_{t}$ be a set of size $|T| \leq 100 \delta \theta n$. Let $0.01 \leq$ $z \leq 1$. Let $\mathcal{N}_{T}$ be the set of all clauses $b$ of $\Phi^{t}$ such that $|N(b) \cap T| \geq z|N(b)|$ and 
$0.1 \theta k \leq|N(b)| \leq 10 \theta k$. Then (i) implies that

$$
\sum_{b \in \mathcal{N}_{T}}|N(b)| \leq \frac{1.009}{z}|T|+10^{-6} \delta \theta n .
$$

Furthermore, (ii) yields

$$
\begin{aligned}
\sum_{b:|N(b) \cap T| \geq z|N(b)|}|N(b)| & \leq \sum_{b:|N(b)| \notin[0.1 \theta k, 10 \theta k]}|N(b)|+\sum_{b \in \mathcal{N}_{T}}|N(b)| \\
& \leq 1.009|T| / z+2 \cdot 10^{-5} \delta \theta n,
\end{aligned}
$$

as desired.

\subsection{Establishing Q4}

We remember condition $\mathbf{Q 4}$ :

For any set $T \subset V_{t}$ of size $|T| \leq 10 \delta \theta n$, the linear operator $\Lambda_{T}: \mathbf{R}^{V_{t}} \rightarrow \mathbf{R}^{V_{t}}$,

$$
\Gamma=\left(\Gamma_{y}\right)_{y \in V_{t}} \mapsto\left\{\sum_{b \in N_{\leq 1}(x, T)} \sum_{y \in N(b) \backslash\{x\}} 2^{-|N(b)|} \operatorname{sign}(x, b) \operatorname{sign}(y, b) \Gamma_{y}\right\}_{x \in V_{t}}
$$

has norm $\left\|\Lambda_{T}\right\|_{\square} \leq \delta^{4} \theta n$.

Lemma 4.20. For all $1 \leq t \leq \hat{t}$ we have

$$
\mathrm{P}\left[\boldsymbol{\Phi}^{t} \text { satisfies } \mathbf{Q 4} \mid \boldsymbol{\Phi} \text { is tame }\right] \geq 1-\exp \left[-10 \Delta_{t}-0.01 \theta n\right] .
$$

To prove Lemma 4.20, we work with the probability distribution $\boldsymbol{\Phi}_{\text {seq }}$ (sequence of $m$ independent clauses). Let $\mathcal{M}$ be the set of all indices $l \in[m]$ such that the $l$ th clause $\boldsymbol{\Phi}_{\text {seq }}(l)$ does not contain any of the variables $x_{1}, \ldots, x_{t}$ positively. In this case, $\boldsymbol{\Phi}_{\text {seq }}(l)$ is still present in the decimated formula $\boldsymbol{\Phi}_{\text {seq }}^{t}$ (with all occurrences of $\neg x_{1}, \ldots, \neg x_{t}$ eliminated, of course). For each $l \in \mathcal{M}$, let $\mathcal{L}(l)$ be the number of literals in $\boldsymbol{\Phi}_{\text {seq }}(l)$ whose underlying variable is in $V_{t}$. We may assume without loss of generality that for any $l \in \mathcal{M}$ the $\mathcal{L}(l)$ "leftmost" literals $\boldsymbol{\Phi}_{\text {seq }}(l, i), 1 \leq i \leq \mathcal{L}(l)$, are the ones with an underlying variable from $V_{t}$.

Let $T \subset V_{t}$. Analyzing the operator $\Lambda_{T}$ directly is a little awkward. Therefore, we will decompose $\Lambda_{T}$ into a sum of several operators that are easier to investigate. For any $0.1 \theta k \leq L \leq 10 \theta k, 1 \leq i<j \leq L, l \in \mathcal{M}$, and any distinct $x, y \in V_{t}$, we define

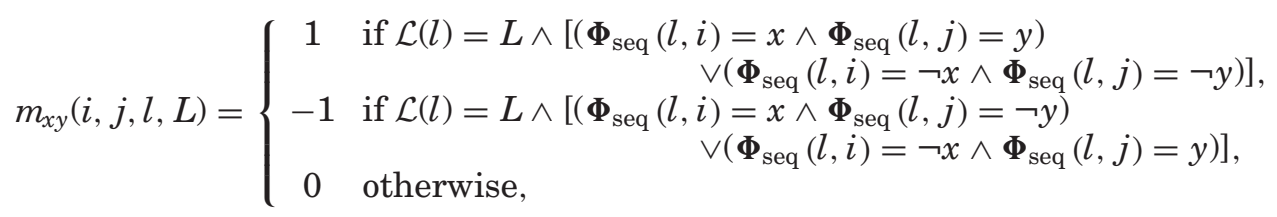

while we let $m_{x x}(i, j, l, L)=0$. Moreover, for $x, y \in V_{t}$ we let

$$
m_{x y}(i, j, L)=\sum_{l \in \mathcal{M}} m_{x y}(i, j, l, L) .
$$

For a variable $x \in V_{t}$, we let $\mathcal{N}(x, T)$ be the set of all $l \in \mathcal{M}$ such that $0.1 \theta k \leq \mathcal{L}(l) \leq 10 \theta k$ and the clause $\boldsymbol{\Phi}_{\text {seq }}(l)$ contains at most one literal whose underlying variable is in $T \backslash x$. Moreover, for $l \in \mathcal{M}$, let $\mathcal{N}(x, l)$ be the set of all variables $y \in V_{t} \backslash\{x\}$ that occur in clause 
$\boldsymbol{\Phi}_{\text {seq }}(l)$ (either positively or negatively). We are going to analyze the operators:

$$
\Lambda_{T}^{i j L}: \mathbf{R}^{V_{t}} \rightarrow \mathbf{R}^{V_{t}}, \quad \Gamma=\left(\Gamma_{y}\right)_{y \in V_{t}} \mapsto\left\{\sum_{l \in \mathcal{N}(x, T)} \sum_{y \in \mathcal{N}(x, l)} 2^{-L} m_{x y}(i, j, l, L) \Gamma_{y}\right\}_{x \in V_{t}} .
$$
have

Lemma 4.21. For any $0.1 \theta k \leq L \leq 10 \theta k, 1 \leq i<j \leq L$ and for any set $T \subset V_{t}$, we

$$
\mathrm{P}\left[\left\|\Lambda_{T}^{i j L}\right\|_{\square} \leq \delta^{5} \theta n\right] \geq 1-\exp (-\theta n) .
$$

Proof. The proof is based on Fact 1.9. Fix two sets $A, B \subset V_{t}$. For each $l \in \mathcal{M}$ and any $x, y \in V_{t}$ the two $0 / 1$ random variables

$$
\sum_{(x, y) \in A \times B} \max \left\{m_{x y}(i, j, l, L), 0\right\}, \quad \sum_{(x, y) \in A \times B} \max \left\{-m_{x y}(i, j, l, L), 0\right\}
$$

are identically distributed because the clause $\boldsymbol{\Phi}_{\text {seq }}(l)$ is chosen uniformly at random. In effect, the two random variables

$$
\begin{aligned}
\mu(A, B) & =\sum_{l \in \mathcal{M}} \sum_{(x, y) \in A \times B} \mathbf{1}_{l \in \mathcal{N}(x, T)} \max \left\{m_{x y}(i, j, l, L), 0\right\}, \\
v(A, B) & =\sum_{l \in \mathcal{M}} \sum_{(x, y) \in A \times B} \mathbf{1}_{l \in \mathcal{N}(x, T)} \max \left\{-m_{x y}(i, j, l, L), 0\right\}
\end{aligned}
$$

are identically distributed. Furthermore, both $\mu(A, B)$ and $\nu(A, B)$ are sums of independent Bernoulli variables because the clauses $\left(\boldsymbol{\Phi}_{\text {seq }}(l)\right)_{l \in[m]}$ are mutually independent.

We need to estimate the mean $\mathrm{E}(\mu(A, B))=\mathrm{E}(\nu(A, B))$. Because each of the clauses $\boldsymbol{\Phi}_{\text {seq }}(l)$ is chosen uniformly, for each $l \in[m]$ we have

$$
\mathrm{P}[l \in \mathcal{M} \wedge \mathcal{L}(l)=L]=\left(\begin{array}{l}
k \\
L
\end{array}\right) \theta^{L}(1-\theta)^{k-L} 2^{L-k} .
$$

Therefore,

$$
\begin{aligned}
\mathrm{E}[\mu(A, B)+v(A, B)] & \leq m\left(\begin{array}{l}
k \\
L
\end{array}\right) \theta^{L}(1-\theta)^{k-L} 2^{L-k} \\
& =\frac{2^{L} \rho \theta n}{L}\left(\begin{array}{l}
k-1 \\
L-1
\end{array}\right) \theta^{L-1}(1-\theta)^{k-L} \quad\left[\text { as } m=2^{k} \rho / k\right] \\
& \leq \frac{2^{L} \rho \theta n}{L} .
\end{aligned}
$$

Hence, Lemma 1.8 (the Chernoff bound) yields

$$
\begin{aligned}
\mathrm{P}[|\mu(A, B)-\mathrm{E}(\mu(A, B))| & \left.>10 \sqrt{2^{L} \rho / L} \cdot \theta n\right] \\
& =\mathrm{P}\left[|\nu(A, B)-\mathrm{E}(\nu(A, B))|>10 \sqrt{2^{L} \rho / L} \cdot \theta n\right] \\
& =16^{-\theta n} .
\end{aligned}
$$

Hence, by the union bound

$$
\begin{array}{r}
\mathrm{P}\left[\exists A, B \subset V_{t}: \max \{|\mu(A, B)-\mathrm{E}(\mu(A, B))|,|\nu(A, B)-\mathrm{E}(\nu(A, B))|\}>10 \sqrt{2^{L} \rho / L} \cdot \theta n\right] \\
\leq 2 \cdot 4^{\theta n} \cdot 16^{-\theta n} \leq \exp (-\theta n) .
\end{array}
$$


Thus, with probability $\geq 1-\exp (-\theta n)$, we have

$$
\begin{aligned}
\left\langle\Lambda_{T}^{i j L} \mathbf{1}_{B}, \mathbf{1}_{A}\right\rangle & =2^{-L}(\mu(A, B)-v(A, B)) \\
& \leq 2^{-L}(|\mu(A, B)-\mathrm{E}[\mu(A, B)]|+|v(A, B)-\mathrm{E}[v(A, B)]|) \\
& \leq \theta n \cdot 20 \sqrt{\frac{\rho}{L 2^{L}}} \leq 0.01 \delta^{5} \theta n,
\end{aligned}
$$

because $L \geq 0.1 k \theta, \theta k \geq \ln (\rho) / c^{2}$, and $\delta=\exp (-c k \theta)$. Finally, the assertion follows from Fact 1.9.

Corollary 4.22. With probability at least $1-\exp (-0.1 \theta n)$, the random formula $\boldsymbol{\Phi}_{\mathrm{seq}}^{t}$ has the following property:

Let $T \subset V_{t}$ and let

$$
\Lambda_{T}^{\prime}=\sum_{0.1 \theta k \leq L \leq 10 \theta k} \sum_{j=1}^{L} \sum_{1 \leq i<j} \Lambda_{T}^{i j L} .
$$

Then, $\left\|\Lambda_{T}^{\prime}\right\|_{\square} \leq \delta^{4.9} \theta n$.

Proof. By Lemma 4.21 and the union bound, we have

$$
\mathrm{P}\left[\exists T, i, j, L:\left\|\Lambda_{T}^{i j L}\right\|_{\square}>\delta^{5} \theta n\right] \leq(10 \theta k)^{3} 2^{\theta n} \cdot \exp (-\theta n) \leq \exp (-0.1 \theta n) .
$$

Furthermore, if $\left\|\Lambda_{T}^{i j L}\right\|_{\square} \leq \delta^{5} \theta n$ for all $i, j, L$, then by the triangle inequality

$$
\left\|\Lambda_{T}^{\prime}\right\|_{\square} \leq(10 \theta k)^{3} \delta^{5} \theta n \leq \delta^{4.9} \theta n \quad[\text { as } \delta=\exp (-c k \theta)],
$$

as claimed.

Proof of Lemma 4.20. We observe that for $(x, y) \in V_{t} \times V_{t}$ the $(x, y)$ entries of the matrices $\Lambda_{T}$ and $\Lambda_{T}^{\prime}$ differ only if either $x$ or $y$ occurs in a redundant clause. Consequently, $\mathbf{Q 0}$ ensures that $\left\|\Lambda_{T}^{\prime}-\Lambda_{T}\right\|_{\square}=o(n)$. Therefore, Fact 4.1, Equation (84), and Corollary 4.22 imply that $\boldsymbol{\Phi}^{t}$ satisfies $\mathbf{Q} 4$ with the desired probability.

\section{ACKNOWLEDGMENT}

I thank two anonymous referees for their careful reading and their valuable feedback. Moreover, I owe a debt of gratitude to Samuel Hetterich for pointing out several minor corrections that he came across in the course of his work [Hetterich 2016].

\section{REFERENCES}

D. Achlioptas and Y. Peres. 2004. The threshold for random $k$-SAT is $2^{k} \ln 2-O(k)$. Journal of the AMS 17 (2004), 947-973.

D. Achlioptas, A. Naor, and Y. Peres. 2005. Rigorous location of phase transitions in hard optimization problems. Nature 435 (2005), 759-764.

D. Achlioptas. 2009. Handbook of Satisfiability. IOS Press, 245-270.

D. Achlioptas and G. Sorkin. 2000. Optimal myopic algorithms for random 3-SAT. Proc. 41st FOCS (2000), 590-600.

M. Bayati, D. Shah, and M. Sharma. 2008. Max-product for maximum weight matching: Convergence, correctness, and LP duality. IEEE Transactions on Information Theory 54 (2008), 1241-1251.

H. Bethe. 1935. Statistical theory of superlattices. Proceedings of the Royal Society of London A 150 (1935), 552-558.

A. Braunstein, M. Mézard, and R. Zecchina. 2005. Survey propagation: An algorithm for satisfiability. Random Structures and Algorithms 27 (2005), 201-226. 
V. Chvatal and B. Reed. Mick gets some (the odds are on his side). 1992. Where the really hard problems are. In Proceedings of the 33th FOCS. 620-627.

A. Coja-Oghlan and D. Achlioptas. 2008. Algorithmic barriers from phase transitions. In Proceedings of the 49th FOCS. 793-802.

A. Coja-Oghlan. 2010. A better algorithm for random k-SAT. SIAM Journal of Computing 39 (2010), 28232864.

J. Franco and M. T. Chao. 1990. Probabilistic analysis of a generalization of the unit-clause literal selection heuristic for the $k$-satisfiability problem. Inform. Sci. 51 (1990), 289-314.

R. G. Gallager. 1963. Low-Density Parity Check Codes. MIT Press.

S. Hetterich. 2016. Analysing survey propagation guided decimation on random formulas. In Proceedings of the 43rd ICALP. 65:1-65:12.

E. Lalas, A. Kaporis, and L. Kirousis. 2006. The probabilistic analysis of a greedy satisfiability algorithm. Random Structures and Algorithms 28 (2006), 444-480.

H. Levesque, D. Mitchell, and B. Selman. 1992. Hard and easy distribution of SAT problems. In Proceedings of the 10th AAAI. 459-465.

M. Mézard and A. Montanari. 2009. Information, Physics and Computation. Oxford University Press.

M. Molloy, D. Achlioptas, and P. Beame. 2004. Exponential bounds for DPLL below the satisfiability threshold. In Proceedings of the 15th SODA. 139-140.

A. Pachon-Pinzon and A. Coja-Oghlan. 2012. The decimation process in random $k$-SAT. SIAM Journal of Discrete Mathematics 26 (2012), 1471-1509.

J. Pearl. 1988. Probabilistic Reasoning in Intelligent Systems: Networks of Plausible Inference. Morgan Kaufmann Publishers.

F. Ricci-Tersenghi, G. Semerjian, L. Zdeborova, F. Krzakala, and A. Montanari. 2007. Gibbs states and the set of solutions of random constraint satisfaction problems. Proceedings of the National Academy of Sciences 104 (2007), 10318-10323.

F. Ricci-Tersenghi, R. Marino, and G. Parisi. 2015. The backtracking survey propagation algorithm for solving random K-SAT problems. arXiv 1508.05117 (2015).

A. Ruciński, S. Janson, and T. Łuczak. 2000. Random Graphs. Wiley.

B. Selman and C. P. Gomes. 2002. Satisfied with physics. Science 297 (2002), 784-785.

B. Selman and S. Kirkpatrick. 1994. Critical behavior in the satisfiability of random Boolean expressions. Science 264 (1994), 1297-1301.

B. Selman, L. Kroc, and A. Sabharwal. 2009. Message-passing and local heuristics as decimation strategies for satisfiability. In Proceedings of the 24th SAC. 1408-1414.

G. Semerjian and F. Ricci-Tersenghi. 2009. On the cavity method for decimated random constraint satisfaction problems and the analysis of belief propagation guided decimation algorithms. Journal of Statistical Mechanics (2009), P09001.

G. Semerjian, A. Montanari, and F. Ricci-Tersenghi. 2007b. Solving constraint satisfaction problems through belief propagation-guided decimation. In Proceedings of the 45th Allerton.

D. Shah and A. Montanari. 2007a. Counting good truth assignments of random k-SAT formulae. In Proceedings of the 18th SODA. 1255-1264.

M. Sudan and D. Gamarnik. 2003. The satisfiability threshold of random 3-SAT is at least 3.52. IBM Research Report RC22942 (2003).

M. Sudan and D. Gamarnik. 2014. Performance of the survey propagation-guided decimation algorithm for the random NAE-K-SAT problem. arXiv 1402.0052 (2014).

S. Suen and A. Frieze. 1996. Analysis of two simple heuristics on a random instance of $k$-SAT. Journal of Algorithms 20 (1996), 312-355.

N. Sun, J. Ding, and A. Sly. 2015. Proof of the satisfiability conjecture for large $k$. In Proceedings of the 47th STOC. 59-68.

W. Taylor, P. Cheeseman, and B. Kanefsky. 1991. Where the really hard problems are. In Proceedings of the IJCAI. 331-337.

P. Tetali, A. Montanari, and R. Restrepo. 2011. Reconstruction and clustering in random constraint satisfaction problems. SIAM Journal of Discrete Math 25 (2011), 771-808. DOI : http://dx.doi.org/10.1137/ 090755862

E. Upfal, A. Broder, and A. Frieze. 1993. On the satisfiability and maximum satisfiability of random 3-CNF formulas. In Proceedings of the 4th SODA. 322-330.

R. Urbanke and A. Montanari. 2007c. Modern Coding Theory: The Statistical Mechanics and Computer Science Point of View. 
R. Urbanke, T. Richardson, and A. Shokrollahi. 2001. Design of capacity-approaching irregular low-density parity check codes. IEEE Transactions on Information Theory 47 (2001), 619-637.

D. Vilenchik, A. Coja-Oghlan, and E. Mossel. 2009. A spectral approach to analyzing belief propagation for 3-coloring. Combinatorics, Probability and Computing 18 (2009), 881-912.

D. Vilenchik, U. Feige, and E. Mossel. 2013. Complete convergence of message passing algorithms for some satisfiability problems. Theory of Computing 9 (2013), 617-651.

M. Wainwright, E. Maneva, and E. Mossel. 2007. A new look at survey propagation and its generalizations. Journal of the ACM 54 (2007).

Y. Wei, D. Gamarnik, and D. Shah. 2012. Belief propagation for min-cost network flow: Convergence \& correctness. Operations Research 60 (2012), 410-428.

R. Zecchina, M. Mézard, and G. Parisi. 2002. Analytic and algorithmic solution of random satisfiability problems. Science 297 (2002), 812-815.

R. Zecchina, S. Mertens, and M. Mézard. 2006. Threshold values of random $K$-SAT from the cavity method. Random Structures, and Algorithm 28 (2006), 340-373.

Received April 2013; revised September 2016; accepted September 2016 